\title{
Grouping Sentences as a Better Language Unit for Extractive Text Summarization
}

\author{
Mengyun Cao and Hai Zhuge* \\ Institute of Computing Technology, Chinese Academy of Sciences, University of Chinese Academy of Sciences, Beijing, China \\ Laboratory of Cyber-Physical-Social Intelligence, Guangzhou University, China \\ Laboratory of Semantics, Knowledge and Data, Aston University, Birmingham, UK \\ *Corresponding author: Hai Zhuge (e-mail:h.zhuge@aston.ac.uk).
}

\begin{abstract}
Most existing methods for extractive text summarization aim to extract important sentences with statistical or linguistic techniques and concatenate these sentences as a summary. However, the extracted sentences are usually incoherent. The problem becomes worse when the source text and the summary are long and based on logical reasoning. The motivation of this paper is to answer the following two related questions: What is the best language unit for constructing a summary that is coherent and understandable? How is the extractive summarization process based on the language unit? Extracting larger language units such as a group of sentences or a paragraph is a natural way to improve the readability of summary as it is rational to assume that the original sentences within a larger language unit are coherent. This paper proposes a framework for group-based text summarization that clusters semantically related sentences into groups based on Semantic Link Network (SLN) and then ranks the groups and concatenates the top-ranked ones into a summary. A two-layer SLN model is used to generate and rank groups with semantic links including the is-part-of link, sequential link, similar-to link, and cause-effect link. The experimental results show that summaries composed by group or paragraph tend to contain more key words or phrases than summaries composed by sentences; and, summaries composed by groups contain more key words or phrases than those composed by paragraphs, especially when the average length of source texts is from 7,000 words to 17,000 words, which is the usual length of scientific papers. Further, we compare seven clustering algorithms for generating groups and propose five strategies for generating groups with the four types of semantic links.
\end{abstract}

Keywords: Text Summarization; Semantic Link Network; Clustering; Natural Language Processing.

\section{Introduction}

Automatic text summarization is an important research direction in the area of natural language processing. It is a desirable approach to deal with the rapid growth of online texts. A good summary should convey the core ideas of the source texts fluently while minimizing the redundancy [1]. Text summarization tasks can be classified into single-document or multi-document summarization tasks according to the number of source texts, and can be classified into generic or theme-focused (also known as query-focused) summarization tasks according to whether the summarization concerns all themes or specific themes of the source text. Text summarization methods are generally divided into extractive and abstractive [2]. Extractive methods usually rank sentences by analyzing statistical and linguistic features such as word/phrase frequency, part-of-speech of words/phrases, position of sentence, and so on, and extract top-k ranked sentences to compose a summary [3]. Abstractive methods attempt to discover the main events (or key words/phrases) and their relations in the source text based on background lexicon and linguistic knowledge, and rephrase these main events (or key words/phrases) into new sentences with possible new words or phrases [1]. 
A Sentence is a basic language unit that represents a complete meaning. Within a meaningful text, there are many semantic links between sentences such as cause-effect, sequential, example-of, and so on. However, most extractive summarization methods rank sentences rarely considering the semantic interdependence between sentences. Therefore, these methods essentially extract each sentence in isolation, and the summary composed by these isolated sentences usually has low readability because the context and the original semantic links between the selected sentences are lost. Figure 1 gives an example of losing context and semantic links in the summary composed by top-ranked sentences. Readers cannot know what "they" and "the learned function" refer to by just reading the generated summary, because sentences $\mathrm{s}_{26}$ and $\mathrm{s}_{37}$ that contain the correct referents are not extracted to generate the summary. We call these anaphors whose referents cannot be found from the text as dangling anaphors. Sentences $\mathrm{s}_{27}$ and $\mathrm{s}_{109}$ are the effects of two cause-effect links in the source text, and they are extracted to generate the summary while their corresponding causes (i.e. $\mathrm{s}_{26}$ and $\mathrm{s}_{108}$ ) are not extracted due to their low ranks. This situation forms incomplete or incorrect representation in the generated summary, which confuses readers.

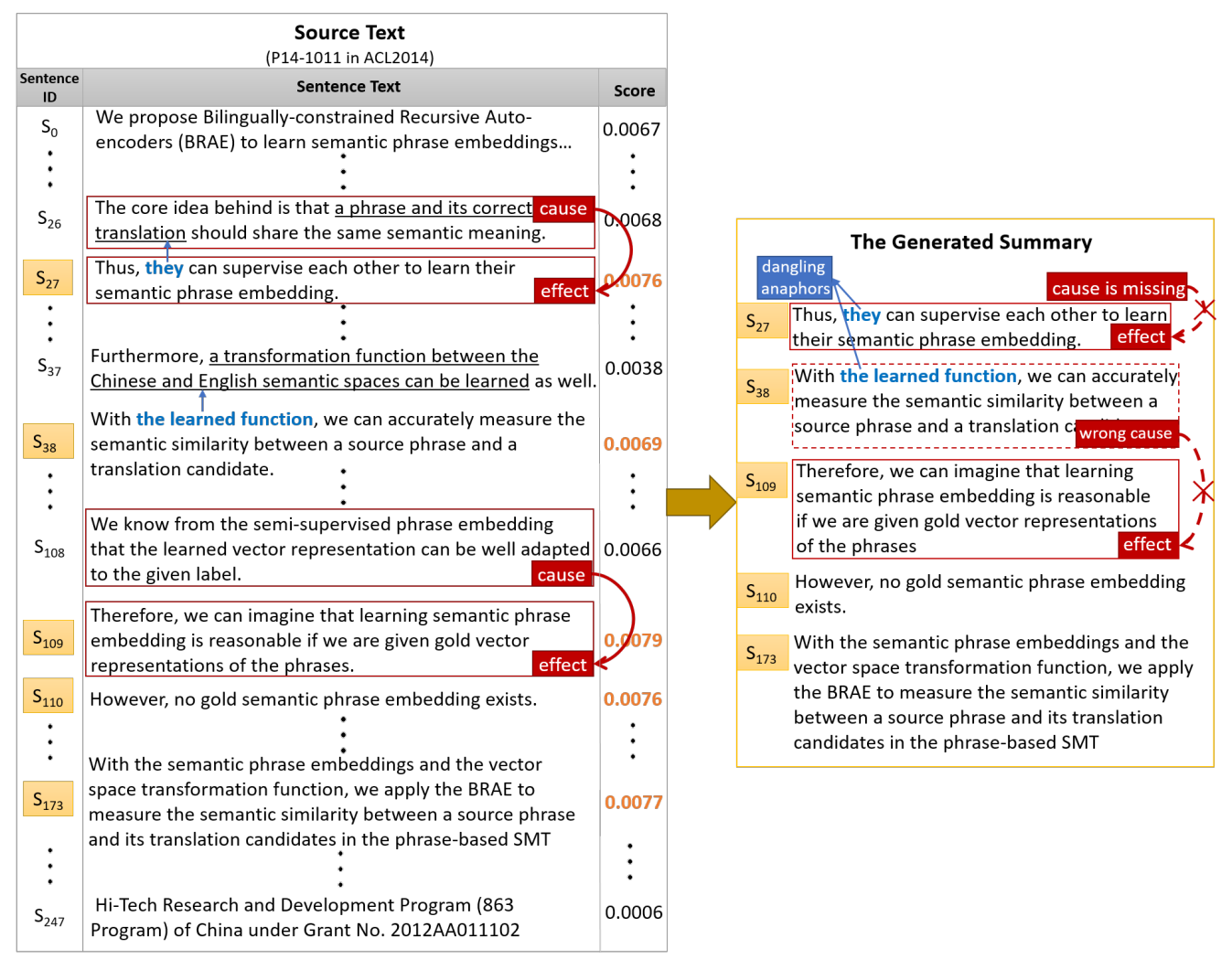

Figure 1. An example of losing context and semantic links in the summary composed by top-ranked sentences.

Since sentences in the source text are coherent, extracting a larger language unit that retains the original order of sentences is a natural way to improve the coherence of the generated summary.

A paragraph is a language unit where its sentences are organized by the writer(s) to convey a complete meaning to readers. It is reasonable to assume that sentences within a paragraph of a published text are coherent. However, ranking and extracting paragraphs directly may reduce the core ideas of the source text contained in the generated summary because: 1) a paragraph may discuss multiple themes but not all of them are related to the core ideas; 2 ) some sentences within a paragraph are just for helping complete the description of a theme (e.g., the use of examples), which should not be included in the summary; 3 ) discussion of a theme sometimes crosses paragraphs, i.e., several paragraphs may commonly render a theme of the source text. Therefore, ranking and extracting appropriate language units with a flexible range is more suitable to generate summaries. 
A group is a language unit that contains a group of semantically related sentences on the same theme and the size of group is smaller than paragraph. There are different ways to organize a group. This paper focuses on using the following four types of semantic links to cluster sentences into groups.

- Is-part-of link, reflecting the relation between a system and its components. Within texts, sentences contribute to render the content of paragraphs, which render the content of sections, which further render the content of the whole text [1].

- Sequential link, reflecting a linear order of operating a process. Sentences are organized in sequential order so that readers can understand the content in the same order of representation. Some semantic links such as cause-effect link and temporal link depend on sequential order. Using the sequential link to organize group can reduce dangling anaphors within each group since the referents of most anaphors are located in nearby sentences [4].

- Similar-to link, reflecting similarity between things. It can be used to detect similar sentences when organizing group.

- Cause-effect link, reflecting causal relation between things. Two sentences linked by cause-effect link render the same theme. Therefore, a basic rule for organizing a group is that the sentence representing cause and the sentence representing the effect should be put into the same group.

The left-hand part of Figure 2 shows an example of grouping sentences according to the sequential link, similar-to link, and cause-effect link: the sentences of a group are consecutive within the source text; sentences $\mathrm{S}_{37}$ and $\mathrm{s}_{38}$ are clustered into group $\mathrm{g}_{26}$ with the lexical similar-to link; sentences $\mathrm{s}_{26}$ and $\mathrm{s}_{27}$ are clustered into group $\mathrm{g}_{19}$ and sentences $\mathrm{s}_{108}$ and $\mathrm{s}_{109}$ are clustered into group $\mathrm{g}_{85}$ with cause-effect links. The "paragraph ID $\leftarrow$ sentence ID" shows the is-part-of link between sentence and paragraph, but it is not used as a clustering constraint in this example, so groups like $g_{26}$ can contain sentences that belong to different paragraphs. The right-hand part of Figure 2 shows the summary generated by ranking these groups and extracting top-ranked groups. Comparing with the summary generated by ranking sentences in Figure 1, the summary generated by ranking groups remains more context and semantic links.

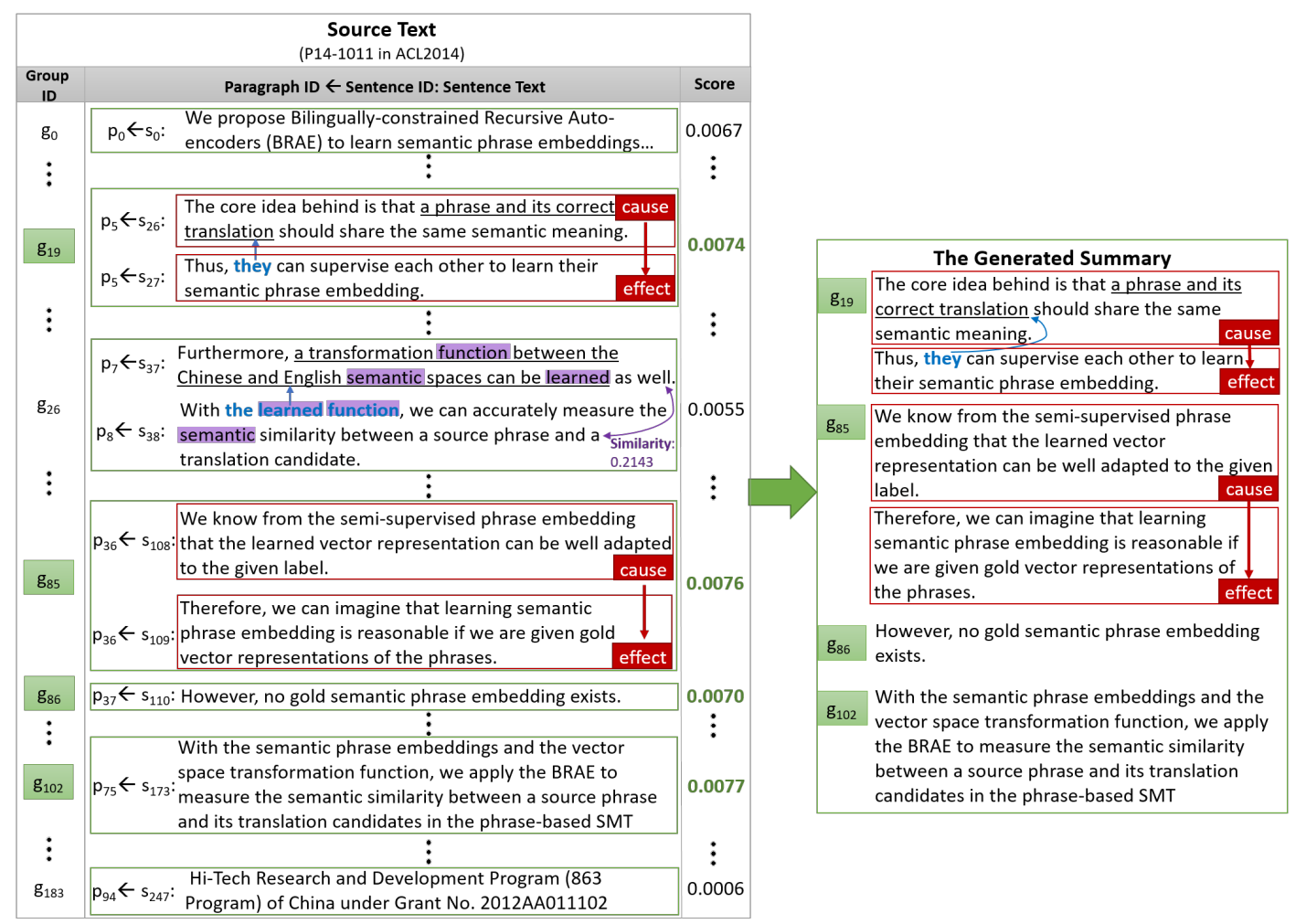

Figure 2. An example of generating a summary by ranking and extracting groups of semantically related sentences. 
However, if a group contains too many sentences or the sentences within a group are less semantically linked, the summary generated by ranking and extracting such groups will contain more sentences that are not relevant to the theme of the source text. Therefore, it is necessary to investigate the suitable semantic links and the suitable clustering algorithm to generate groups.

\section{Group-based Text Summarization}

\subsection{Framework}

The group-based text summarization framework as shown in Figure 3 consists of four components: the Semantic Link Network (in short SLN $[1,5]$ ) of sentences and paragraphs, the algorithm for generating groups, the SLN of groups, and the algorithm for ranking groups. Users can define their own approaches by selecting different semantic links to construct the SLNs and selecting (or designing) algorithms to generate and rank groups.

The framework provides a new approach to improve the current extractive text summarization by designing appropriate algorithms for generating groups connected by various semantic links.

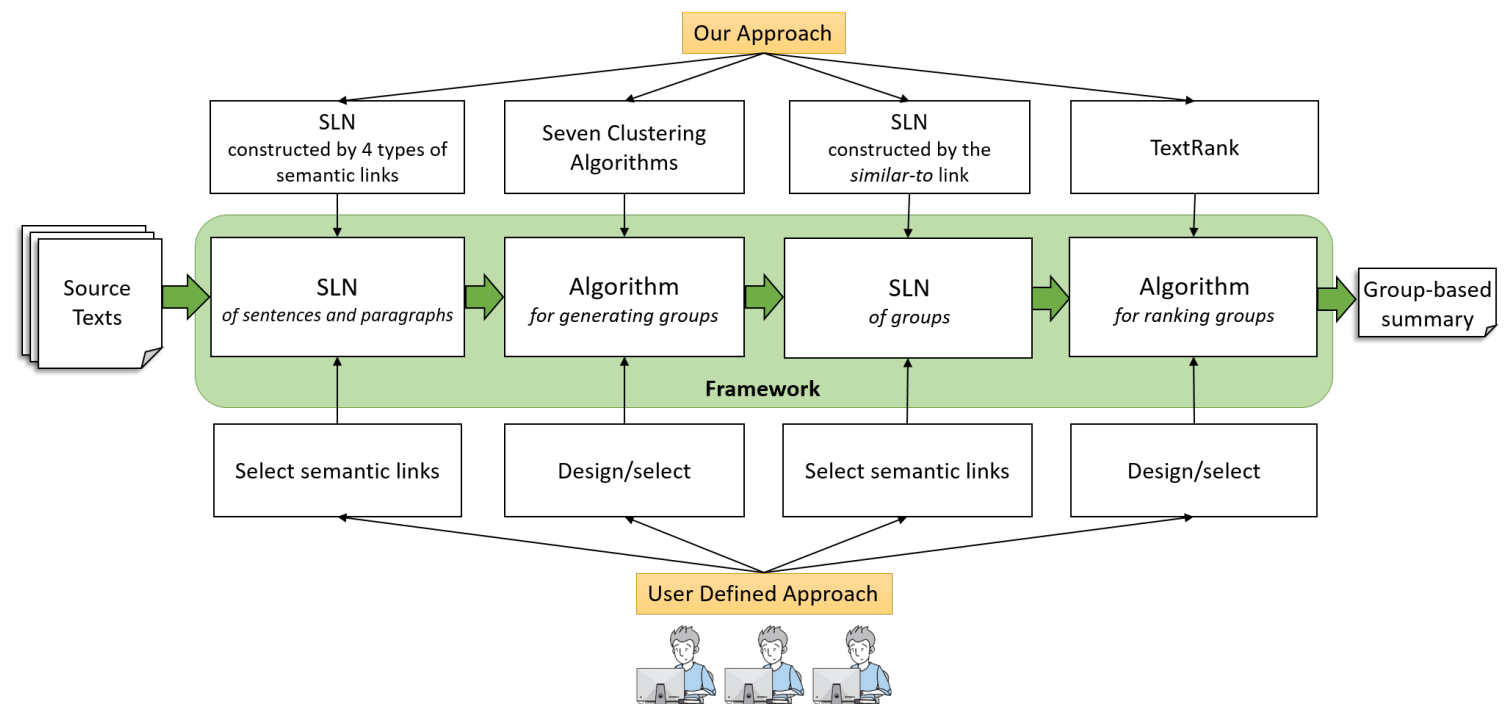

Figure 3. The framework for group-based text summarization.

\subsection{The General Architecture of Our Approach}

Basing on the framework for group-based text summarization, we design an approach that uses the is-partof, sequential, similar-to, and cause-effect links to construct the SLN of sentences and paragraphs, selects one from the seven clustering algorithms we designed to generate groups, uses the similar-to link to construct the SLN of groups, and adopts the TextRank algorithm ([6]) to rank groups.

Figure 4 depicts the general architecture of this study. The middle of the figure is the model we designed. The top and bottom are the two baseline models that generate summaries by ranking sentences or paragraphs. The following four steps of the two-layer SLN model summarizing a source text correspond to the four components of the framework for group-based text summarization:

- Step 1: Constructing the first-layer SLN. The model splits the source text into paragraphs and sentences (nodes) connected by is-part-of links (in blue color), sequential links (in yellow color), similar-to links (in purple color; a thicker line represents higher similarity), and cause-effect links (in red color) to form the first-layer SLN.

- Step 2: Clustering sentences into groups. We designed seven clustering algorithms based on different combinations of the four types of semantic links. The model adopts one of the algorithms to perform on the first-layer SLN for generating groups. 
- Step 3: Constructing the second-layer SLN. The model takes the groups generated in Step 2 as nodes and connects these nodes through similar-to links to form the second-layer SLN, which is also called the simSLN of group (sim-SLN is an SLN that contains only similar-to links).

- Step 4: Ranking groups and then extracting top-ranked groups to compose a summary.

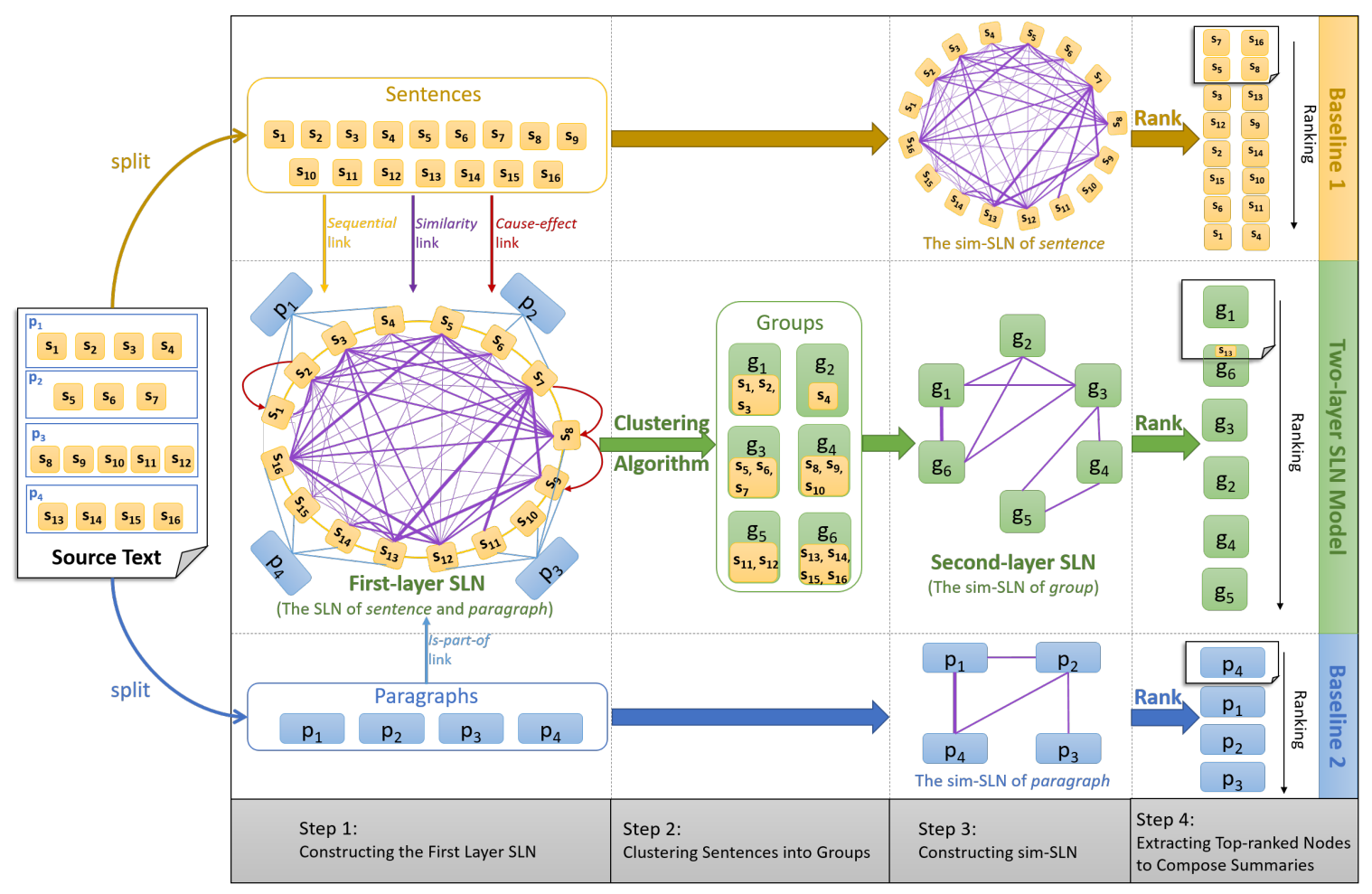

Figure 4. The general architecture of our approach.

\section{Experiments}

\subsection{Aim and method}

To verify the performance of group in generating summaries for long source texts, we make single-document summarization on scientific papers to compare the group-based summary generated by our two-layer SLN model with the sentence-based summary and paragraph-based summary generated by the two baseline models. Just as the Step 3 and Step 4 in Figure 4, the two baseline models summarize a source text by constructing the sim-SLN of sentence or paragraph, performing the TextRank algorithm to rank sentences or paragraphs, and composing a summary with the top-ranked sentences or paragraphs.

To investigate the effects of the four types of semantic links in generating and ranking groups, we further conducted experiments on the two-layer SLN model by using different clustering algorithms and using different types of similar-to links to construct the sim-SLN.

The seven clustering algorithms implemented for generating groups according to different combinations of the is-part-of link, sequential link, similar-to link, and cause-effect link are introduced in Appendix B. Each clustering algorithm is named according to the semantic links it uses, namely Seq+pb/npb, SimSIZE, SimTHR, CE $+p b / n p b, C E S i m, \operatorname{SeqSim}+p b / n p b$, and CESeqSim $+p b / n p b$.

We implemented six similarity metrics to calculate the weight of the similar-to link between two language units, including the lexical-based JCD metric, the embedding-based AVG, SIF and USE metrics, and the synsets-based LIN and WUP metrics. The details of the six similarity metrics are introduced in Appendix A. Each SLN instance at most contains one type of similar-to link.

The explicit cause-effect links contain causal cue words/phrases such as 'because' and 'due to'. Implicit cause-effect links have no causal cue words/phrases, and readers can infer implicit cause-effect links by analyzing the sentences with their background knowledge. The two-layer SLN model uses explicit cause-effect 
links to generate groups, because placing sentences of explicit cause-effect links into different groups could lead to incorrect (or incomplete) representation. We use the pattern-based algorithm [7] to discover the explicit cause-effect link between any pair of consecutive sentences.

\subsection{Experimental Datasets}

One dataset ACL-all contains 173 ACL2014 conference papers downloaded from the ACL Anthology. The other dataset AI-all contains 372 papers from the Artificial Intelligence journal from 1994 to 2018. To study the influence of paper length on the performance of extracting different language units to generate summary, the $A C L$-all dataset is divided into two subsets and the $A I$-all dataset is divided into six subsets. Table 1 shows the details of the two datasets and the eight sub-datasets, in which the column "Average Length" shows the average number of words in each sub-dataset.

We regard texts in abstract, conclusion and introduction of a scientific paper as three kinds of standard summaries. When the kind of standard summary is decided, the text in the standard summary is excluded from the automatic summarization process, and the number of words in the standard summary is regarded as the upper limit of the length of the generated summary. In Table 1, columns "Abstract", "Conclusion" and "Introduction" show the average number of words in each kind of standard summaries on different subdatasets. We can generate longer summaries by simply using longer standard summaries.

Table 1. The details of the experimental datasets.

\begin{tabular}{|c|c|c|c|c|c|c|}
\hline Datasets & Subsets & The Number of Papers & Average Length & Abstract & Conclusion & Introduction \\
\hline \multirow{2}{*}{$A C L$-all } & $A C L$-short & 85 short papers & 2,823 & 99 & 127 & 464 \\
\hline & ACL-long & 88 long papers & 5,494 & 128 & 209 & 607 \\
\hline \multirow{6}{*}{ AI-all } & AI-less 10 & $\begin{array}{l}59 \text { papers less than } 10,000 \\
\text { words }\end{array}$ & 7,762 & 169 & 272 & 924 \\
\hline & $A I-1013$ & $\begin{array}{l}57 \text { papers between } 10,000 \\
\text { and } 13,000 \text { words }\end{array}$ & 11,396 & 184 & 374 & 1271 \\
\hline & $A I-1316$ & $\begin{array}{l}70 \text { papers between } 13,000 \\
\text { and } 16,000 \text { words }\end{array}$ & 14,516 & 201 & 600 & 1132 \\
\hline & $A I-1619$ & $\begin{array}{l}65 \text { papers between } 16,000 \\
\text { and } 19,000 \text { words }\end{array}$ & 17,201 & 205 & 542 & 1405 \\
\hline & $A I-1925$ & $\begin{array}{l}60 \text { papers between } 19,000 \\
\text { and } 25,000 \text { words }\end{array}$ & 21,566 & 198 & 596 & 1573 \\
\hline & AI-more 25 & $\begin{array}{l}61 \text { papers more than } \\
25,000 \text { words }\end{array}$ & 32,768 & 218 & 686 & 1893 \\
\hline
\end{tabular}

\subsection{Four Metrics for Comparing Summaries}

ROUGE evaluation metrics, containing ROUGE-N $(N \in\{1,2,3,4\})$ and ROUGE-L, are widely used to evaluate the lexical similarity between the generated summary and the standard summary [8]. Since the words or phrases used in standard summaries can be viewed as key words/phrases that reflect the core ideas of the source text, we design four metrics based on the ROUGE scores to compare the amount of key words/phrases contained in different kinds of generated summaries.

We connect the scores of ROUGE-1, ROUGE-2, ROUGE-3, ROUGE-4, and ROUGE-L to form a ROUGE vector for each kind of the generated summaries. Let $\boldsymbol{R}_{\boldsymbol{s}}=\left\{R 1_{s}, R 2_{s}, R 3_{s}, R 4_{s}, R L_{s}\right\}$ be the ROUGE vector for the sentence-based summary, where $R i_{s}$ is the average F-score of ROUGE- $i(i \in\{1,2,3,4, L\})$. Similarly, $\boldsymbol{R}_{\boldsymbol{p}}$ and $\boldsymbol{R}_{\boldsymbol{g}}$ are the ROUGE vectors for the paragraph-based summary and the group-based summary. Let $\boldsymbol{R}_{\boldsymbol{s}}$ be the benchmark for comparison and $\boldsymbol{R}$ be the ROUGE vector of the summary to be compared, the four metrics we designed are shown below:

- L2 metric

$$
L 2\left(\boldsymbol{R} \| \boldsymbol{R}_{\boldsymbol{s}}\right)=\sqrt[2]{\sum_{i \in\{1,2,3,4, L\}}(R i)^{2}}-\sqrt[2]{\sum_{i \in\{1,2,3,4, L\}}\left(R i_{S}\right)^{2}}
$$




\section{- Increase metric}

$$
\operatorname{Increase}\left(\boldsymbol{R} \| \boldsymbol{R}_{\boldsymbol{s}}\right)=\sum_{i \in\{1,2,3,4, L\}}\left(R i-R i_{s}\right)
$$

\section{- Increase\% metric}

ROUGE-1 and ROUGE-L scores are usually much higher than ROUGE-3 and ROUGE-4 scores. In the $L 2$ metric and the Increase metric, a significant increase on $R 3$ or $R 4$ can be easily offset by a tiny decrease on $R 1$ or $R L$. Therefore, Increase \% is proposed for showing the average increment on each dimension.

$$
\text { Increase } \%\left(\boldsymbol{R} \| \boldsymbol{R}_{\boldsymbol{s}}\right)=\frac{\sum_{i \in\{1,2,3,4, L\}} \frac{\left(R i-R i_{S}\right)}{R i_{S}} * 100 \%}{5}
$$

\section{- Diverge metric}

Referring to Kullback-Leibler divergence which measures how a probability distribution diverges from the expected one [9], we propose Diverge $\left(\boldsymbol{R} \| \boldsymbol{R}_{\boldsymbol{s}}\right)$ to measure the deviation of the ROUGE vector $\boldsymbol{R}$ from the basis ROUGE vector $\boldsymbol{R}_{\boldsymbol{s}}$.

$$
\operatorname{Diverge}\left(\boldsymbol{R} \| \boldsymbol{R}_{\boldsymbol{s}}\right)=\sum_{i \in\{1,2,3,4, L\}} R i * \ln \frac{R i}{R i_{s}}
$$

The value range of the $L 2$ metric or the Increase metric is $[-5,5]$, the value range of the Increase $\%$ metric is $[-100 \%, 100 \%]$, and the value range of the Diverge metric is $[-\infty,+\infty]$. If the values of more than two metrics are greater than zero, then we say that the summaries of $\boldsymbol{R}$ contain more key words/phrases than the summaries of $\boldsymbol{R}_{\boldsymbol{s}}$.

\subsection{Comparison based on Four Metrics}

\subsubsection{Preliminary Comparison}

Our previous work [10] also used the framework for group-based text summarization to automatically generate summaries for each paper of the $A C L$-all dataset. It used the SeqSim $+p b(J C D, 0.06)$ algorithm to generate groups and used the JCD-type similar-to links to construct the sim-SLN.

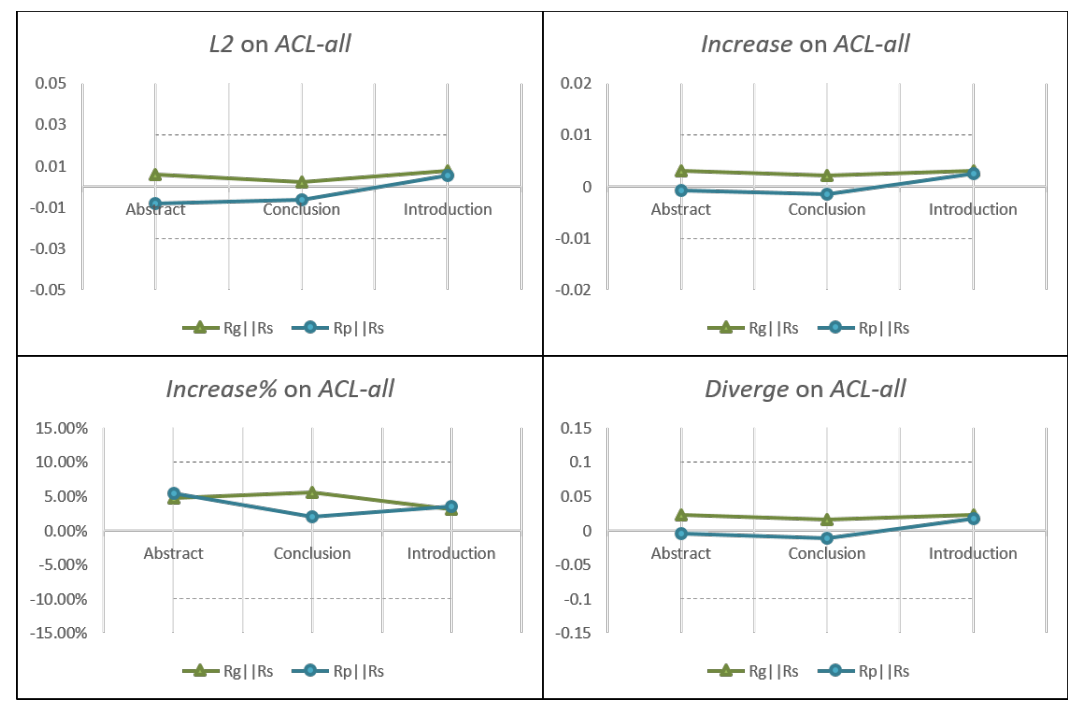

Figure 5. The values of the four metrics on the $A C L$-all dataset when using $\operatorname{SeqSim}+p b(J C D, 0.06)$ and JCD-type sim-SLN.

Figure 5 shows the values of the four metrics obtained from the experiments of the previous work. Series $\boldsymbol{R}_{\boldsymbol{g}}|| \boldsymbol{R}_{\boldsymbol{s}}$ compares the group-based summary with the sentence-based summary. If $\boldsymbol{R}_{\boldsymbol{g}} \| \boldsymbol{R}_{\boldsymbol{s}}$ is greater than zero on more than two metrics, we say the group-based summary contains more key words/phrases than the sentence-based summary. If $-0.025 \leq L 2\left(\boldsymbol{R}_{\boldsymbol{g}} \| \boldsymbol{R}_{\boldsymbol{s}}\right) \leq 0.025,-0.01 \leq$ Increase $\left(\boldsymbol{R}_{\boldsymbol{g}} \| \boldsymbol{R}_{\boldsymbol{s}}\right) \leq 0.01, \quad-10 \% \leq$ Increase\% $\left(\boldsymbol{R}_{g}|| \boldsymbol{R}_{\boldsymbol{s}}\right) \leq 10 \%$ and $-0.1 \leq$ Diverge $\left(\boldsymbol{R}_{\boldsymbol{g}}|| \boldsymbol{R}_{\boldsymbol{s}}\right) \leq 0.1$ (the value interval between two dotted lines in each 
sub-figure of Figure 5), we say that the performance of group is similar to the performance of sentence. The $\boldsymbol{R}_{p}|| \boldsymbol{R}_{s}$ compares the paragraph-based summary with the sentence-based summary in the same way. If the value of $\boldsymbol{R}_{g}|| \boldsymbol{R}_{s}$ is greater than the value of $\boldsymbol{R}_{p} \| \boldsymbol{R}_{s}$ on more than two metrics, we say that the group-based summary contains more key words/phrases than the paragraph-based summary.

For generating summaries for each paper of the $A I$-all dataset, we first use the same experimental settings used in the previous work to generate and rank groups. Figure 6 shows the values of the four metrics on the $A I$-all dataset. From Figure 5 and Figure 6, we find that the group-based summary contains more key words/phrases than sentence-based summary on both the ACL-all and the AI-all datasets. The paragraphbased summary contains more key words/phrases than the sentence-based summary on the AI-all dataset, but on the ACL-all dataset paragraph performs better than sentence only when Introduction is used as standard summary.

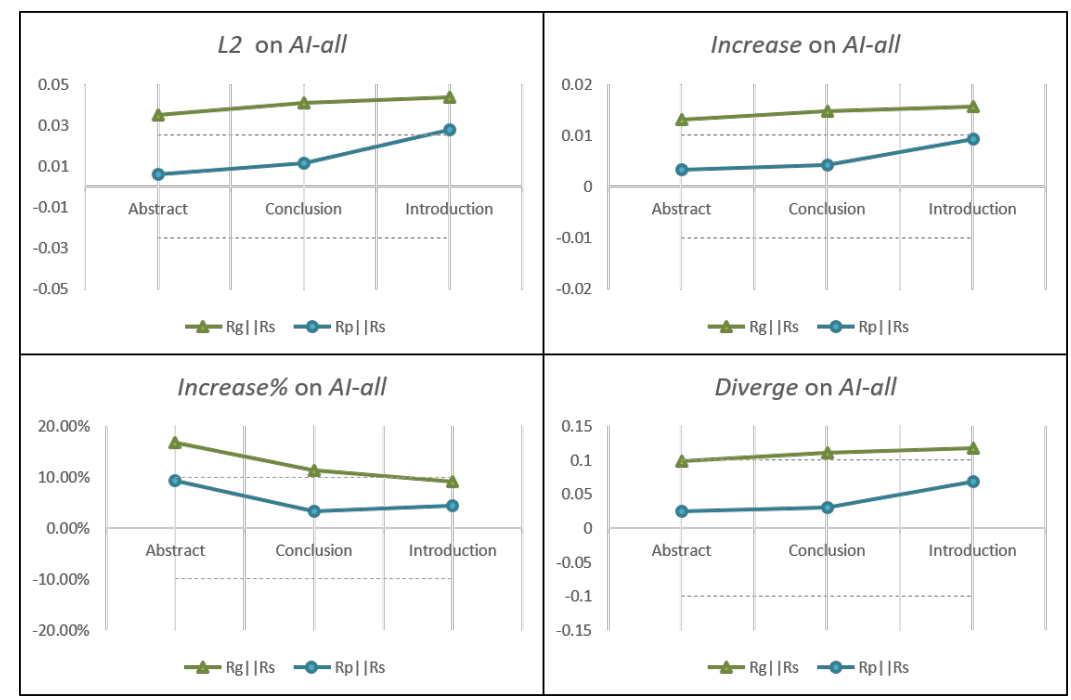

Figure 6. The values of the four metrics on the AI-all dataset when using $\operatorname{SeqSim}+p b(J C D, 0.06)$ and JCD-type sim-SLNs.

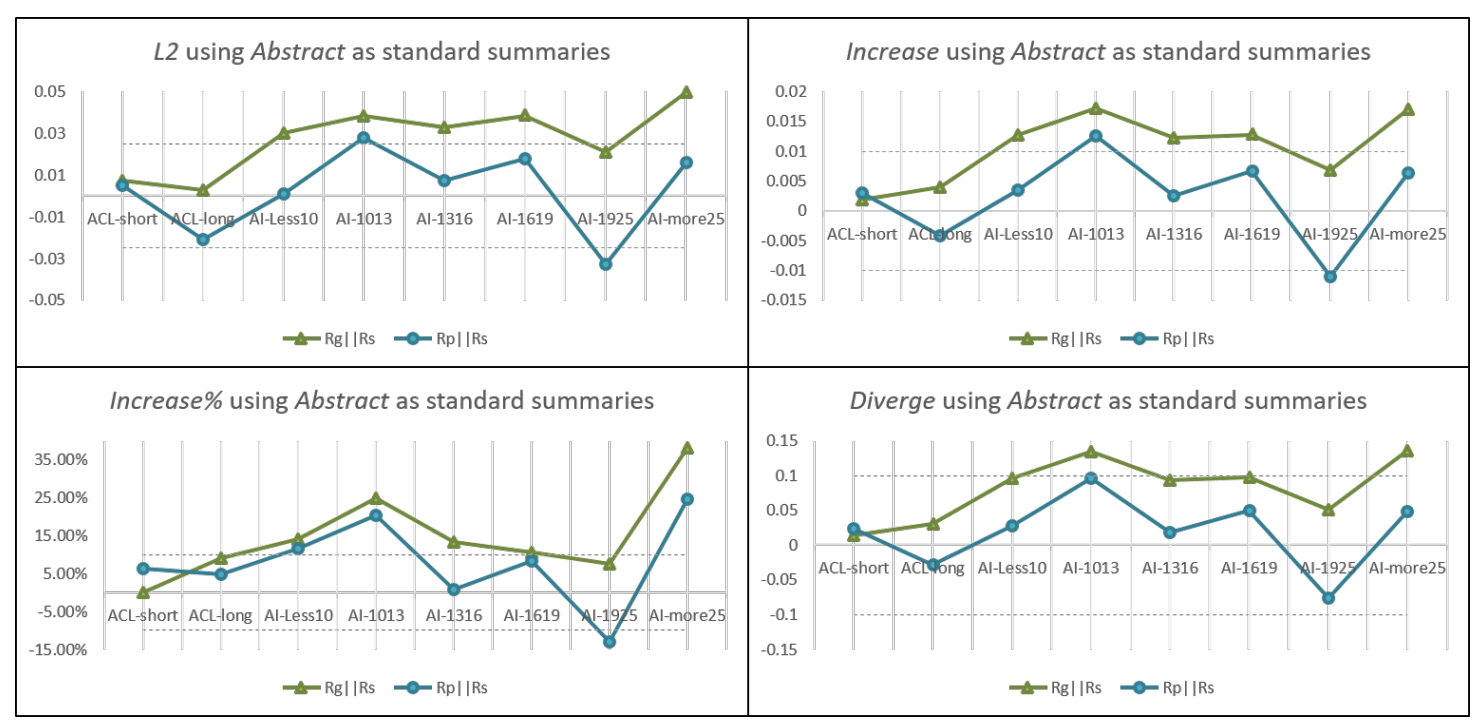

Figure 7. The values of the four metrics on each sub-dataset when using $\operatorname{SeqSim}+p b(J C D, 0.06)$ for generating groups and using JCDtype sim-SLN for ranking language units.

Figure 7 shows the values of the four metrics on each sub-dataset when using the Abstract as the standard summary, from which we see that the performance of group in generating summaries is significantly improved when the average length of the source texts is more than 7000 words. (Figure C-1 in Appendix C.1 
gives the values of the four metrics on each sub-dataset when using the Conclusion or the Introduction as the standard summary, from which we draw the same conclusion).

Besides, we randomly sample some sentence-based, group-based and paragraph-based summaries for manually evaluating the readability of these summaries. The readability statistics shown in Appendix D verifies the assumption that the summary composed by larger language units has higher readability than the sentencebased summary.

\subsubsection{Further Comparison}

We further test the performance of group in generating summaries by changing the type of similar-to links used for constructing the sim-SLN, since different similar-to links guide TextRank to rank language units differently and thus cause different groups (sentences or paragraphs) are extracted to compose the summary. We still use the SeqSim + $p b(J C D, 0.06)$ algorithm to generate groups, but use the JCD-type, AVG-type, SIFtype, USE-type, LIN-type, and WUP-type similar-to links in turn to construct the sim-SLN for ranking sentences, groups, and paragraphs.

Table C-1 to Table C-4 in Appendix C.2 shows the values of each metric on the ACL-all dataset and AI-all dataset when using different types of sim-SLN. Table 2 gives the statistics of the four metrics counted from Table C-1 to Table C-4. As shown in Table 2, when using different sim-SLNs to rank language units for extracting top-ranked ones to generate summaries, the group-based approach or the paragraph-based approach performs at least as good as the sentence-based approach with a probability greater than $97 \%$, and the group-based approach or the paragraph-based approach performs better than sentence with a probability greater than $63 \%$. This means that the group-based or paragraph-based summary tends to contain more key words/phrases than sentence-based summary even if we change the types of sim-SLN for ranking language units.

Table 2. The statistics of the four metrics in different ranges on ACL-all and AI-all.

\begin{tabular}{|c|c|c|c|}
\hline \multirow{2}{*}{ Performance } & \multirow{2}{*}{$\begin{array}{l}\text { The range of value of the } \\
\text { four metrics }\end{array}$} & \multicolumn{2}{|c|}{ Larger Language Units } \\
\hline & & group & paragraph \\
\hline \multirow{4}{*}{$\begin{array}{l}\text { At least similar to } \\
\text { sentence }\end{array}$} & $L 2\left(R \| R_{s}\right)>-0.025$ & $97.22 \%$ & $97.22 \%$ \\
\hline & Increase $\left(R \| R_{s}\right)>-0.01$ & $100 \%$ & $97.22 \%$ \\
\hline & Increase $\%\left(R \| R_{s}\right)>-10 \%$ & $100 \%$ & $97.22 \%$ \\
\hline & $\operatorname{Diverge}\left(R \| R_{s}\right)>-0.1$ & $100 \%$ & $100 \%$ \\
\hline \multirow{4}{*}{ Better than sentence } & $L 2\left(R \| R_{s}\right)>0$ & $75 \%$ & $63.89 \%$ \\
\hline & Increase $\left(R \| R_{s}\right)>0$ & $77.78 \%$ & $66.67 \%$ \\
\hline & Increase $\%\left(R \| R_{s}\right)>0$ & $83.33 \%$ & $77.78 \%$ \\
\hline & $\operatorname{Diverge}\left(R \| R_{s}\right)>0$ & $77.78 \%$ & $69.44 \%$ \\
\hline \multirow{4}{*}{$\begin{array}{l}\text { Significantly better } \\
\text { than sentence }\end{array}$} & $L 2\left(R \| R_{s}\right)>0.025$ & $27.78 \%$ & $25 \%$ \\
\hline & Increase $\left(R \| R_{s}\right)>0.01$ & $19.44 \%$ & $22.22 \%$ \\
\hline & Increase $\%\left(R \| R_{s}\right)>10 \%$ & $22.22 \%$ & $30.56 \%$ \\
\hline & $\operatorname{Diverge}\left(R \| R_{s}\right)>0.1$ & $13.89 \%$ & $13.89 \%$ \\
\hline
\end{tabular}

In Table 2, the probability that "group is significantly better than sentence" is slightly less than the probability that "paragraph is significantly better than sentence". This is probably due to the existence of other concluding paragraphs within a scientific paper except for the text in Abstract, Introduction and Conclusion, and the sentences in a concluding paragraph are usually concluding sentences. If a concluding paragraph is extracted to compose a summary, the paragraph-based summary will contain more concluding sentences than the group-based summary since each group generated by the SeqSim $+p b(J C D, 0.06)$ algorithm contains fewer sentences than the paragraph to which it belongs. However, given that the probability that "group is better than sentence" is $9 \%$ more than the probability that "paragraph is better than sentence" on average, we can still conclude that group tends to outperform both sentence and paragraph. 


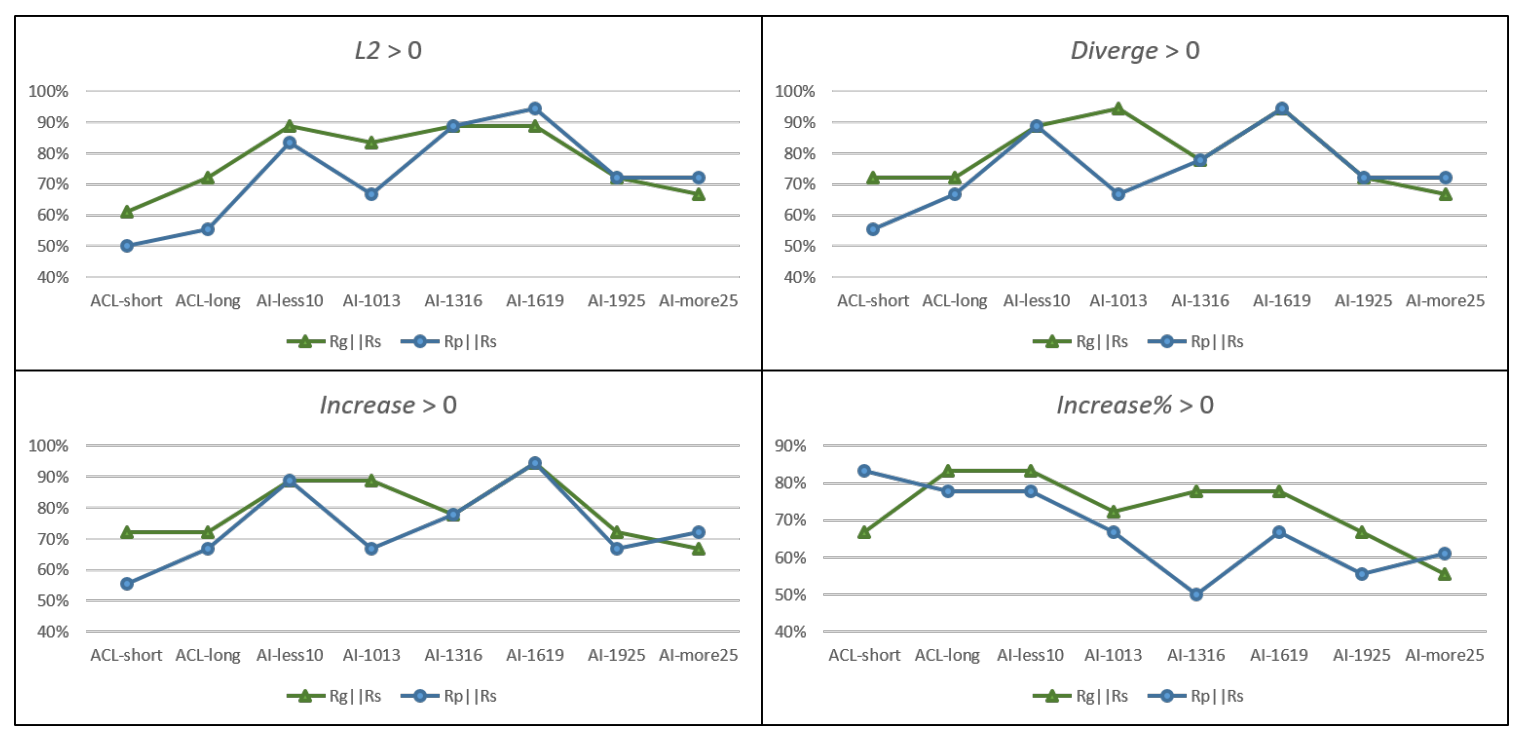

Figure 8 . The percentage of cases where the four metrics are greater than zero on each sub-dataset.

Table C-5 to Table C-8 in Appendix C.2 shows the values of each metric on all the sub-datasets when using different types of sim-SLN. Figure 8 shows the percentages of cases where the four metrics are greater than zero on each sub-dataset in Table C-5 to Table C-8. From Figure 8 we can see that, when the source text becomes longer, the percentages of cases where $\boldsymbol{R}_{g} \| \boldsymbol{R}_{s}$ is greater than zero first improves, then remains stable, and finally declines. The sub-datasets on which group has stable performance are AI-less 10, AI-1013, AI1316, AI-1619, so the best range of the average length of source texts for ranking groups to generate summaries is from 7000 words to 17000 words. However, the percentages of cases where $\boldsymbol{R}_{p} \| \boldsymbol{R}_{s}$ is greater than zero varies frequently with the length of the source text, so it is difficult to determine the best range of the average length of source texts for the paragraph-based summary.

Combining the experiment results shown in the previous subsection, we can draw the following two conclusions:

Conclusion 1: summaries composed by group or paragraph tend to contain more key words or phrases than summaries composed by sentence.

Conclusion 2: summaries composed by group contain more key words or phrases than those based on paragraph, especially when the average length of source texts is from 7,000 and 17,000 words.

Figure 9 compares the performance of different types of similar-to links in ranking language units to generate summaries, where $L 2\left(\boldsymbol{R}_{s}\right), L 2\left(\boldsymbol{R}_{\boldsymbol{g}}\right)$, and $L 2\left(\boldsymbol{R}_{\boldsymbol{P}}\right)$ are the L2 norms of the ROUGE-score vectors for the sentence-based, group-based, and paragraph-based summary respectively. We can see that the performance of the embedding-based similar-to link (AVG, SIF or GSE) becomes significantly better than the lexicalbased (JCD) or synsets-based (LIN or WUP) similar-to link when the average length of source texts becomes longer. 


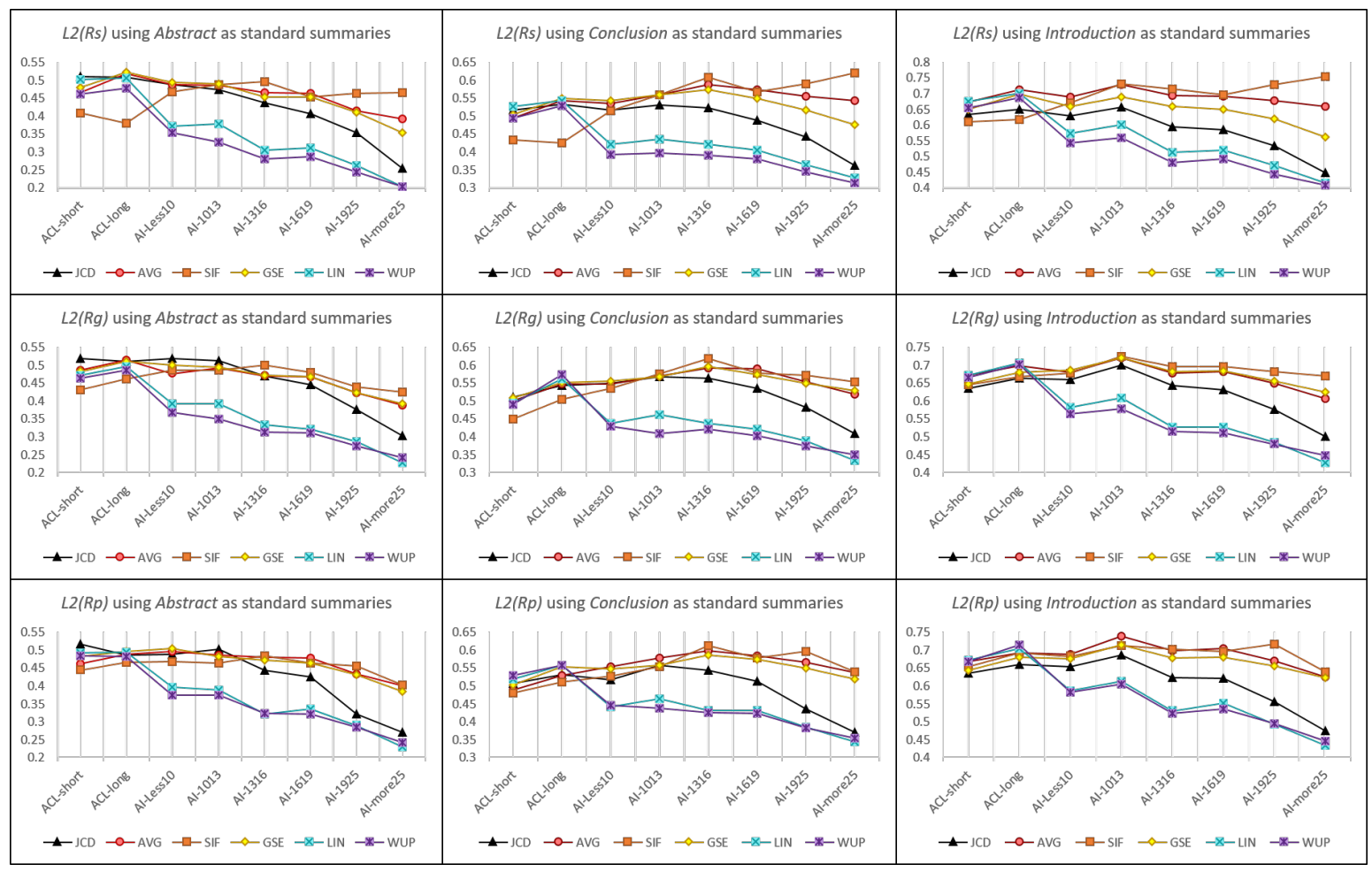

Figure 9. The L2 norms of $R s, R g$ and $R p$ on each sub-dataset when using different types of sim-SLN to rank nodes.

\subsubsection{The Role of Semantic Links in Generating Groups}

We compare the performance of the seven clustering algorithms to investigate the role of the four types of semantic links in generating groups. First, the ' $+p b$ ' and ' $+n p b$ ' modes set in the Seq, CE, SeqSim and CESeqSim algorithms are compared to show the effect of using is-part-of link. Second, for SimSIZE, SimTHR, CESim, SeqSim and CESeqSim that need similar-to links, the lexical-based similarity and the embeddingbased similarity are compared to show the effect of using different types of similar-to links. Third, by setting each algorithm with its suitable paragraph-bounded mode and its suitable type of similar-to links, the seven clustering algorithms are compared to show the best one for generating groups.

Other parameters used in the clustering algorithms are tuned on the AI-less 10 sub-dataset because on $A I$ less 10 the average length of a paper is moderate and the performance of group starts to be significantly better than the performance of sentence in composing summaries. The parameter win_size used in the Seq algorithm (or size used in SimSIZE) means the maximum number of sentences within a generated group. Since a group should contain fewer sentences than a paragraph and more than 95\% paragraphs of the ACL-all and AI-all datasets contain less than 10 sentences, we try with win_size (or size) $=2,3 \ldots 10$ to find the suitable size of group for the Seq algorithm (or the SimSIZE algorithm). The parameter thr used in SimTHR, CESim, SeqSim and CESeqSim algorithms refers to the similarity threshold to merge two language units. Since the weight of a similar-to link ranges from 0 to 1 , we try $t h r=0.01,0.02, \ldots, 0.99$ to find the suitable similarity threshold for each of the four algorithms.

The AVG-type similar-to links are used for constructing the sim-SLNs and the text in the abstract of each paper is used as the standard summary. We only show the results of the Diverge metric here, since the four metrics show the same conclusions on the performance of different kinds of groups. 


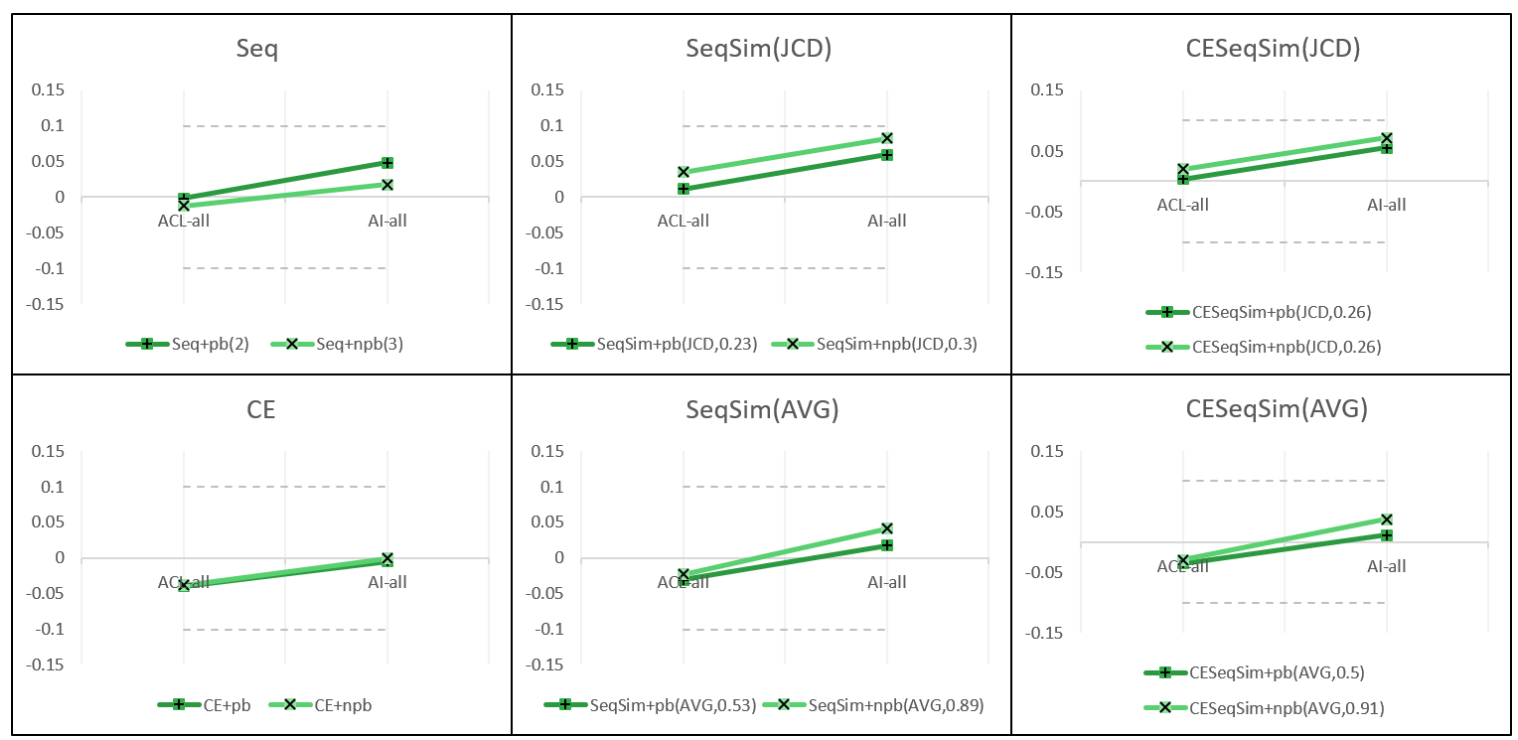

(a) Using ' $+p b$ ' or ' $+n p b$ ' mode in each clustering algorithm.

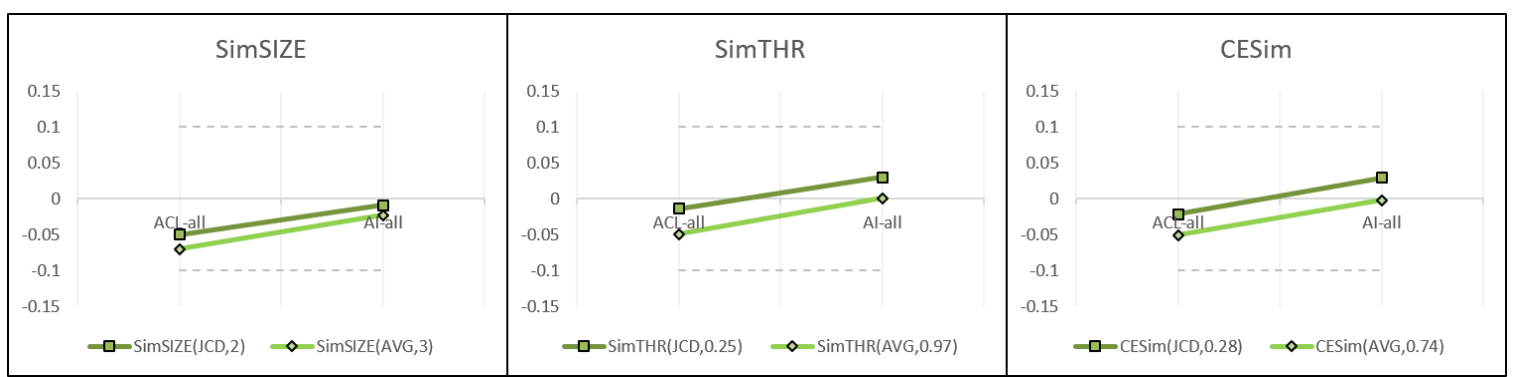

(b) Using JCD-type or AVG-type similar-to links in each clustering algorithm.

Figure 10. The Diverge metric results of each kind of groups on the $A C L$-all and AI-all datasets when using AVG-type sim-SLNs to rank nodes and using Abstract as standard summaries.

Figure 10 shows the Diverge metric of each kind of group on the ACL-all and AI-all datasets, where the series $\operatorname{Seq}+p b(2)$ is short for Diverge $\left(R_{\text {Seq }}+p b(2) \| R_{S}\right)$, and so on. We can see from Figure 10(a) that the Seq, $C E$, SeqSim and CESeqSim algorithms have different performance under the ' $+p b$ ' mode and the ' $+n p b$ ' mode:

- For the $\mathrm{Seq}$ algorithm, the groups generated under the ' $+p b$ ' mode get higher Diverge results than the groups generated under the ' $+n p b$ ' mode. This shows that when only using the sequential links to generate groups, the paragraph-bounded restriction can help reduce the cases that unrelated sentences within consecutive paragraphs are clustered into the same group.

- For the $C E$ algorithm, there is almost no difference between the ' $+p b$ ' mode and the ' $+n p b$ ' mode. This is because only $2.73 \%$ cause-effect links within the $A C L$-all and AI-all datasets cross paragraphs, and fewer of these paragraph-crossing cause-effect links are among the top-ranked groups that are used to compose summaries.

- For the SeqSim and CESeqSim algorithms, the groups generated under the ' $+n p b$ ' mode get higher $\mathrm{Di}$ verge results than the groups generated under the ' $+p b$ ' mode. This phenomenon can be explained in two aspects. (1) Using sequential and similar-to links simultaneously has a similar effect as the paragraphbounded restriction because the first sentence of a paragraph is often dissimilar to the last few sentences of the preceding paragraph. (2) When the first sentence of a paragraph is similar to the last few sentences of the preceding paragraph, this sentence usually plays a role in connecting the preceding paragraph to the paragraph to which it belongs. This makes sense to cluster the last few sentences of the preceding paragraph with this connective sentence into the same group because they usually talk about the same theme. 
From SeqSim and CESeqSim in Figure 10(a) and from Figure 10(b), we can see that using JCD-type similar-to links gets higher Diverge results than using AVG-type similar-to links for the five clustering algorithms that need similar-to links. This shows that the lexical-based similar-to link outperforms the embedding-based similar-to link in generating groups.

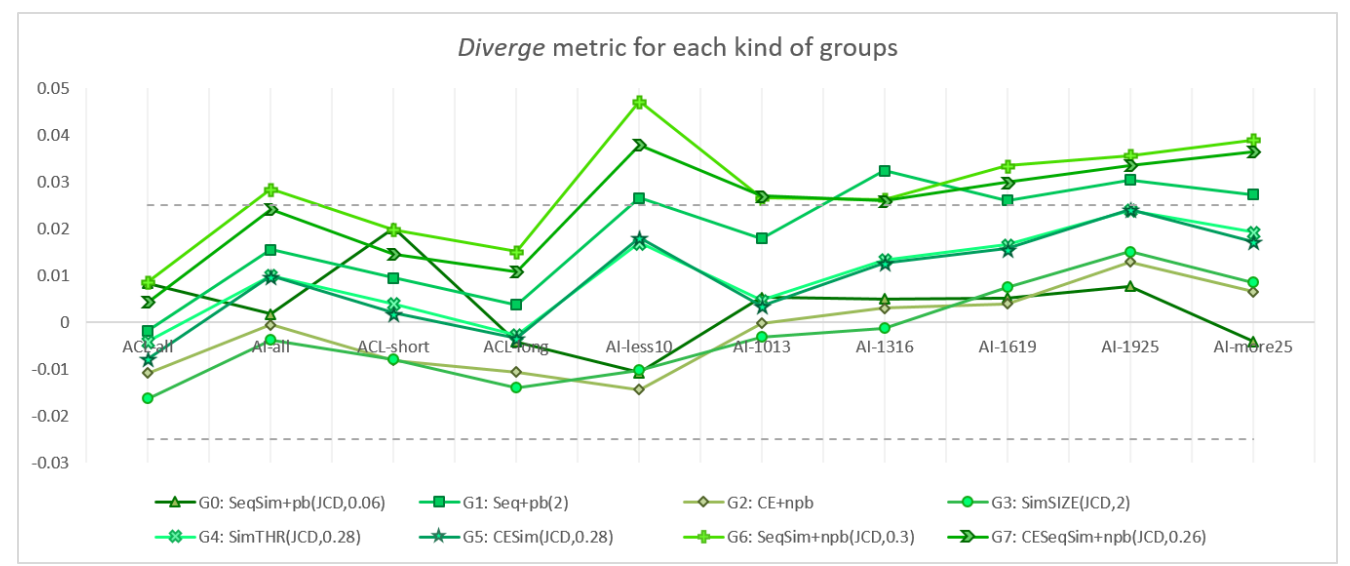

Figure 11. The Diverge metric results of different kinds of groups on all datasets when using AVG-type sim-SLNs to rank nodes and using Abstract as standard summaries.

Figure 11 shows the Diverge metric of using each clustering algorithm when the paragraph-bounded restriction and the type of similar-to links are suitable. The series named as G0: $\operatorname{SeqSim}+p b(J C D, 0.06)$ shows the Diverge results of the clustering algorithm we used in Section 3.4.1 and 3.4.2. As shown in Figure 11, it is a baseline to show the Diverge results of other clustering algorithms (or the SeqSim algorithm under different parameters). From Figure 11 we can see that:

- G6 is superior to G1, G3 and G4. This shows that the sequential link and the similar-to link are quite suitable for being used together to generate groups.

- There is no big difference between G4 and G5 or between G6 and G7, and the Diverge results of G2 are relatively lower than many other clustering algorithms. As shown in Figure 2, clustering sentences that convey a cause-effect link into a group can reduce incorrect or incomplete cause-effect links in the generated summary. This suggests that the cause-effect link can improve the readability of the group-based summary without reducing the number of key words/phrases contained in the summaries.

- G4 is significantly better than G3. This shows that, when using the similar-to link to generate groups, it is better not to limit the number of sentences within each group but to limit the degree of similarity between sentences within each group.

- In previous experiments, we use G0 to generate groups and draw the conclusion that "group outperforms sentence and paragraph in producing extractive summaries". However, both G6 and G7 outperform G0, so this conclusion still holds when using G6 or G7 to generate groups.

- Comparing the Diverge results of G6 or G7 on each sub-dataset, we can find that the group-based summary becomes significantly better than the sentence-based summary when the source text longer than the average length of papers in AI-less 10. Thus, the conclusion that "summaries composed by group contain more core ideas especially when the average length of source texts is longer than 7,000 words" still holds when using G6 or G7 to generate groups.

Based on the above analysis, the SeqSim $+n p b(J C D)$ algorithm and the $C E \operatorname{SeqSim}+n p b(J C D)$ algorithm are the best of the seven clustering algorithms for generating groups. Accordingly, we propose the following strategies for generating groups using the is-part-of, sequential, similar-to and cause-effect links:

Strategy 1: The is-part-of link is a suitable clustering constraint when only using the sequential link to generate groups, while in other cases of using the is-part-of link as a clustering constraint has no effect or even has an adverse effect for generating groups.

Strategy 2: The lexical-based similar-to link is more suitable for generating groups, while the embeddingbased similar-to link is more suitable for ranking groups.

Strategy 3: When only using the similar-to link to generate groups, it is better not to restrict the number of sentences within each group but to restrict the degree of similar-to between sentences within each group. 
Strategy 4: The sequential link is well suitable for being used together with the similar-to link for generating groups.

Strategy 5: The cause-effect link improves the readability of the group-based summary without reducing the amount of key words/phrases contained in the summaries.

The above five strategies complement our proposed framework when the four types of semantic links are used in clustering algorithms.

Appendix C.3 gives more experiment results to support the above strategies.

\section{Related Works}

The Semantic Link Network (SLN) was initially proposed for organizing and operating Web resources in a semantic space $[11,12]$. It has been developed as a systematic theory and method for representing and operating the semantic structure of various complex systems $[5,13,14]$. An instance model of SLN mainly contains semantic nodes, semantic links, rules on semantic links, and operations on nodes and links. Its nodes represent categories of resources and links between nodes represent the semantic relations. Different from the traditional Semantic Net, SLN emphasizes on self-organized "link", on the basic self-organization operations of a complex system, on the emerging semantics [13], and on the automatic discovery of semantic links. The theory and method of SLN have been applied to various application areas, such as building and maintaining Peer-to-Peer networks [15, 16], discovering and representing Knowledge Flow [17], supporting Cyber-Physical-Social Intelligence [18-21], and serving as a methodology for extractive or abstractive text summarization or even for multimedia summarization [1,7, 10, 22-24].

Summarizing citations can be regarded as a kind of group-based extractive summarization, which can also generate coherent and readable summaries. An approach to automatically generating related work through summarizing citations was proposed [22]. But this kind of work is only suitable for scientific papers with citations. The approach proposed in this paper is a general framework that can be applied to not only scientific paper but also other types of texts (especially for those texts with length ranging from 7,000 words to 17,000 words). Reference [23] proposes an extractive text summarization method that constructs SLNs with different types of nodes and links and then performs reinforcement ranking on these SLNs, which verifies the effectiveness of SLN in rendering the core of texts. Reference [7] further verifies the role of SLN in representing the core of scientific papers by investigating the distribution of cause-effect links, the statistics of keywords within cause-effect sentences, and the improvement of the summarization model by adding cause-effect links [23]. Although these two models construct hierarchical SLNs with different language units such as word, sentence, paragraph and section, only sentence is the target language unit for extraction while other language units are used for reinforcing the ranking of sentences through is-part-of links in each step of the iteration.

Most previous extractive summarization models also extract isolated sentences to generate a summary, such as graph-based models [6, 25-28], matrix factorization based models [29, 30], machine learning based models [31-35], neural network based models [36, 37] and so on.

Based on the frame semantics [38], Semantic Role Labelling (SRL) is proposed as a phrase-level semantic analysis task [39]. It is to determine "who did what to whom where when how and why" from a sentence. So it is more suitable for analyzing event-based texts like news. It is limited in ability to infer rich semantic relations between larger language units such as sentences and paragraph. For extractive text summarization application, it is hard to process semantic interdependence such as the cause-effect link between sentences when extracting sentences to compose a summary.

With the development of deep learning, applying deep learning to text summarization become a new approach. Deep learning techniques are especially suitable for processing images. An extractive summarization approach to summarizing multi-media based on deep learning was proposed [40]. Deep learning can also be used to discover semantic link within texts through training process.

Some extractive summarization models cluster sentences into sets according to categories, topics [41-43], similarity [44-46] and adjacency between sentences [47]. Reference [44] aims to generate a citation-based summary for single scientific papers. It first collects suitable citation sentences from other papers that cite the target paper as input, then classifies each citation sentence into five categories (background, problem statement, method, results, and limitations) by a Support Vector Machine classifier, next clusters citation sentences in the same category by performing a community finding on the cosine similarity graph of these sentences, and finally ranks the sentences within each cluster and extracts the top-ranked sentence in each cluster to generate the summary. Some approaches are for enhancing the coverage of topics in the generated summaries. The model introduced in [41] first clusters sentences into topical groups according to the mutual 
reinforcement of term-sentence and the sequences of sentences in the source text, and then selects sentences with the highest saliency scores in each topical group to compose a summary. The model introduced in [42] divides sentences into topical clusters by mutual reinforcements of both terms-sentences and sentences-documents, and ranks topic clusters as well as sentences in each cluster to organize the top-ranked sentences selected from each cluster. Sentences are clustered into topical clusters by performing three classical clustering algorithms on the cosine similarity graph of sentences, then the sentence-to-cluster relation is used to construct a two-layer link graph, and finally a conditional Markov random walk model or a HITS model is performed on the two-layer link graph to rank all sentences [43]. Some approaches aim to decrease redundancy in multi-document summarization by clustering sentences into groups and extracting top-ranked sentences in each group. The model introduced in [45] first generates a summary for every single document, and then cluster sentences of the generated summaries according to syntactic similarity and semantic similarity between sentences. The model introduced in [46] creates a graph with four kinds of links (lexical similarity, semantic similarity, co-reference, and discourse relation) connecting all sentences of the input documents, then performs TextRank on this graph to find the leader sentences which act as the core of clusters, and then uses the Dijkstra's algorithm to cluster each sentence to the nearest cluster. Two sentence-extraction tasks for solving the feature sparseness problem were introduced in [47]. It combines two consecutive sentences into a bi-gram pseudo sentence to enhance the statistical features for selecting salient bi-gram pseudo sentences, separates each selected bi-gram pseudo sentence into two sentences, and selects the salient sentences for producing the final summary. In the above works, sentence is the only language unit for generating summaries.

Our previous work [10] preliminarily studied the performance of the language unit group for extractive summarization. This paper significantly extends the work. Appendix E summarizes the aspects of extensions.

\section{Conclusion}

This paper proposes a group-based extractive text summarization framework by using group to replace sentence as a basic language unit to generate summaries for the first time. It is based on the assumption that summaries composed by larger language units that retain semantic relations between sentences naturally have higher readability. Experiments show that the proposed approach is effective in summarizing texts.

Research also reaches the following results:

- Summaries composed by group or paragraph tend to contain more key words or phrases than summaries composed by sentences.

- Summaries composed by group contain more key words or phrases than those based on paragraph, especially when the average length of source texts is from 7,000 words to 17,000 words.

- Adopting different clustering algorithms can affect the formation of groups, which in turn influences the group-based summary. We suggest the following strategies when the following four types of semantic links are adopted: 1) the is-part-of link is a suitable clustering constraint when only using the sequential link to generate groups, while in other cases of using the is-part-of link as a clustering constraint has no effect or even has adverse effect for generating groups; 2) the lexical-based similar-to link is more suitable for generating groups, while the embedding-based similar-to link is more suitable for ranking groups; 3) when only using the similar-to link to generate groups, it is better not restrict the number of sentences within group but restrict the degree of similar-to link between sentences within group; 4) the sequential link is suitable to be used together with the similar-to link for generating groups; and, 5) the cause-effect link can improve the readability of the group-based summary without reducing the number of key words/phrases contained in the summaries.

This work makes a significant contribution to extractive text summarization and it also verifies the role of semantic links in representing and understanding texts, which are the basis for text summarization.

\section{Acknowledgement}

Professor Hai Zhuge is the corresponding author of this paper. 


\section{References}

[1] H. Zhuge, Multi-Dimensional Summarization in Cyber-Physical Society, Morgan Kaufmann, 2016.

[2] M. Gambhir, V. Gupta, Recent automatic text summarization techniques: a survey, Artificial Intelligence Review, 47 (2017) 1-66.

[3] V. Gupta, G.S. Lehal, A survey of text summarization extractive techniques, Journal of emerging technologies in web intelligence, 2 (2010) 258-268.

[4] S. Lappin, H.J. Leass, An algorithm for pronominal anaphora resolution, Computational Linguistics, 20 (1994) 535-561.

[5] H. Zhuge, The Knowledge Grid: Toward Cyber-Physical Society, World Scientific Publishing Co., 2004 ( $1^{\text {st }}$ edition $), 2012$ ( $2^{\text {nd }}$ edition).

[6] R. Mihalcea, P. Tarau, TextRank: Bringing order into text, in: Proceedings of the Conference on Empirical Methods in Natural Language Processing, 2004, pp. 404-411.

[7] M. Cao, X. Sun, H. Zhuge, The contribution of cause-effect link to representing the core of scientific paper -- The role of Semantic Link Network, PloS one, 13 (2018) e0199303.

[8] C.-Y. Lin, Rouge: A package for automatic evaluation of summaries, in: Proceedings of the ACL-04 Workshop on Text Summarization Branches Out, Barcelona, Spain, 2004, pp. 74-81.

[9] S. Kullback, R.A. Leibler, On information and sufficiency, Annals of Mathematical Statistics, 22 (1951) 79-86.

[10] M. Cao, H. Zhuge, What size of language unit is more appropriate for text summarization?, in: Proceedings of the $14^{\text {th }}$ International Conference on Semantics, Knowledge and Grids (SKG), IEEE, Guangzhou, China, 2018, pp. 196-202.

[11] H. Zhuge, L. Zheng, N. Zhang, X. Li, An automatic semantic relationships discovery approach, in: Proceedings of the $13^{\text {th }}$ International World Wide Web Conference, ACM, 2004, pp. 278-279.

[12] H. Zhuge, The Web Resource Space Model, Springer, 2008.

[13] H. Zhuge, Communities and emerging semantics in Semantic Link Network: Discovery and learning, IEEE Transactions on Knowledge and Data Engineering, 21 (2009) 785-799.

[14] H. Zhuge, Interactive semantics, Artificial Intelligence, 174 (2010) 190-204.

[15] H. Zhuge, J. Liu, L. Feng, X. Sun, C. He, Query routing in a P2P Semantic Link Network, Computational Intelligence, 21 (2005) 197-216.

[16] H. Zhuge, X. Li, Peer-to-Peer in metric space and semantic space, IEEE Transactions on Knowledge and Data Engineering, 19 (2007) 759-771.

[17] H. Zhuge, Discovery of knowledge flow in science, Communications of the ACM, 49 (2006) 101107.

[18] H. Zhuge, Socio-natural thought Semantic Link Network: A method of semantic networking in the Cyber Physical Society, in: Proceedings of $24^{\text {th }}$ IEEE International Conference on Advanced Information Networking and Applications, IEEE, 2010, pp. 19-26.

[19] H. Zhuge, Semantic linking through spaces for Cyber-Physical-Socio Intelligence: A methodology, Artificial Intelligence, 175 (2011) 988-1019.

[20] H. Zhuge, Y. Xing, Probabilistic Resource Space Model for managing resources in Cyber-Physical Society, IEEE Transactions on Service Computing, 5 (2012) 404-421.

[21] H. Zhuge, Cyber-Physical-Social Intelligence on Human-Machine-Nature Symbiosis, Springer, 2019.

[22] J. Chen, H. Zhuge, Automatic generation of related work through summarizing citations, Concurrency and Computation: Practice and Experience, 31 (2019).

[23] X. Sun, H. Zhuge, Summarization of scientific paper through reinforcement ranking on Semantic Link Network, IEEE Access, 6 (2018) 40611-40625.

[24] W. Li, H. Zhuge, Abstractive multi-document summarization based on Semantic Link Network, IEEE Transactions on Knowledge and Data Engineering, (2019) DOI: 10.1109/TKDE.2019.2922957.

[25] G. Erkan, D.R. Radev, LexRank: Graph-based lexical centrality as salience in text summarization, Journal of Qiqihar Junior Teachers College, 22 (2004) 457-479.

[26] X. Zhang, G. Cheng, Y. Qu, Ontology summarization based on rdf sentence graph, in: Proceedings of the $16^{\text {th }}$ international conference on World Wide Web, ACM, 2007, pp. 707-716.

[27] L. Antiqueira, O.N. Oliveira, L.d.F. Costa, M.d.G.V. Nunes, A complex network approach to text summarization, Information Sciences, 179 (2009) 584-599. 
[28] E. Baralis, L. Cagliero, N. Mahoto, A. Fiori, GraphSum: Discovering correlations among multiple terms for graph-based summarization, Information Sciences, 249 (2013) 96-109.

[29] Y. Gong, X. Liu, Generic text summarization using relevance measure and latent semantic analysis, in: Proceedings of the $24^{\text {th }}$ annual international ACM SIGIR conference on Research and development in information retrieval, ACM, New Orleans, Louisiana, USA, 2001, pp. 19-25.

[30] J.-H. Lee, S. Park, C.-M. Ahn, D. Kim, Automatic generic document summarization based on nonnegative matrix factorization, Information Processing \& Management, 45 (2009) 20-34.

[31] D. Shen, J.T. Sun, H. Li, Q. Yang, Z. Chen, Document summarization using conditional random fields, in: Proceedings of the International Joint Conference on Artifical Intelligence, Morgan Kaufmann Publishers Inc., 2007, pp. 2862-2867.

[32] Y. Ouyang, W. Li, R. Zhang, S. Li, Q. Lu, A progressive sentence selection strategy for document summarization, Information Processing \& Management, 49 (2013) 213-221.

[33] M.A. Fattah, A hybrid machine learning model for multi-document summarization, Applied Intelligence, 40 (2014) 592-600.

[34] M. Mendoza, S. Bonilla, C. Noguera, C. Cobos, E. León, Extractive single-document summarization based on genetic operators and guided local search, Expert Systems with Applications, 41 (2014) 4158-4169.

[35] L. Li, K. Zhou, G.R. Xue, H. Zha, Y. Yu, Enhancing diversity, coverage and balance for summarization through structure learning, in: Proceedings of the $18^{\text {th }}$ International Conference on World Wide Web, 2009, pp. 71-80.

[36] M.A. Fattah, F. Ren, GA, MR, FFNN, PNN and GMM based models for automatic text summarization, Computer Speech \& Language, 23 (2009) 126-144.

[37] Z. Cao, F. Wei, L. Dong, S. Li, M. Zhou, Ranking with recursive neural networks and its application to multi-document summarization, in: Proceedings of the $29^{\text {th }}$ AAAI Conference on Artificial Intelligence, AAAI Press, Austin, Texas, 2015, pp. 2153-2159.

[38] C.J. Fillmore, Frame semantics and the nature of language, in: Annals of the New York Academy of Sciences: Conference on the origin and development of language and speech, 1976, pp. 20-32.

[39] D. Gildea, D. Jurafsky, Automatic labeling of semantic roles, Computational linguistics, 28 (2002) 245-288.

[40] J. Chen, H. Zhuge, Extractive summarization of documents with images based on multi-modal RNN, Future Generation Computer Systems, 99 (2019) 186-196.

[41] H. Zha, Generic summarization and keyphrase extraction using mutual reinforcement principle and sentence clustering, in: Proceedings of the $25^{\text {th }}$ annual international ACM SIGIR conference on Research and development in information retrieval, ACM, Tampere, Finland, 2002, pp. 113-120.

[42] L. Yang, X. Cai, Y. Zhang, P. Shi, Enhancing sentence-level clustering with ranking-based clustering framework for theme-based summarization, Information Sciences, 260 (2014) 37-50.

[43] X. Wan, J. Yang, Multi-document summarization using cluster-based link analysis, in: Proceedings of the $31^{\text {st }}$ annual international ACM SIGIR conference on Research and development in information retrieval, ACM, Singapore, 2008, pp. 299-306.

[44] A. Abu-Jbara, D.R. Radev, Coherent citation-based summarization of scientific papers, in: Proceedings of the Association for Computational Linguistics, 2011, pp. 500-509.

[45] V.K. Gupta, T.J. Siddiqui, Multi-document summarization using sentence clustering, in: Proceddings of the $4^{\text {th }}$ International Conference on Intelligent Human Computer Interaction (IHCI), Kharagpur, 2012, pp. 1-5.

[46] R. Ferreira, L.d.S. Cabral, F. Freitas, R.D. Lins, G.d.F. Silva, S.J. Simske, L. Favaro, A multidocument summarization system based on statistics and linguistic treatment, Expert Systems with Applications, 41 (2014) 5780-5787.

[47] Y. Ko, J. Seo, An effective sentence-extraction technique using contextual information and statistical approaches for text summarization, Pattern Recognition Letters, 29 (2008) 1366-1371. 


\section{Appendix A. Six Metrics for Calculating the Similarity between Text Segments}

\section{A.1 Lexical-based Similarity}

If two text language units have many words in common, then they are likely to talk about the same thing. The similarity based on the common words is called the lexical-based similarity. Jaccard distance (JCD) can be used to calculate the proportion of common words in two language units.

Let $s$ be a sentence, $g$ be a group, $p$ be a paragraph and $u \in\{s, g, p\}$. Let $u_{i}=\left\{w_{i, 1}, w_{i, 2}, \ldots w_{i, m}\right\}$ be a language unit containing $m$ words, and $u_{j}=\left\{w_{j, 1}, w_{j, 2}, \ldots w_{j, n}\right\}$ be another language unit containing $n$ words. As shown in formula (1), the JCD-type similarity between $u_{i}$ and $u_{j}$ is calculated by dividing the number of common words to the total number of words.

$$
J C D \operatorname{sim}\left(u_{i}, u_{j}\right)=\frac{\left|\left\{w_{i, 1}, \ldots, w_{i, m}\right\} \cap\left\{w_{j, 1}, \ldots, w_{j, m}\right\}\right|}{m+n}
$$

We remove stop words from two language units before calculating their similarity, because stop words frequently appear in many sentences and convey no meaning. The number of common words is counted by the exact matching of words.

\section{A.2 Embedding-based Similarity}

Word embedding (also known as word representation) has become widely used in various Natural Language Processing tasks. Word embedding models represent each word as a continuous vector by capturing the contexts of this word in a large corpus. The similarity between words can be easily measured by the distance between the vectors of the corresponding words. In the same way, the similarity between language units can be computed by first embedding language units into vectors and then calculating the distance between the embedding vectors.

The GloVe is an unsupervised learning algorithm for obtaining vectors of words by aggregating wordword co-occurrence statistics from a corpus [48], and it has become a standard approach for embedding words. However, there is no standard approach for embedding sentences, groups or paragraphs yet. We implement two unsupervised models and one pre-trained model in this paper for embedding language units into vectors.

\section{- Average (AVG)}

AVG is an unsupervised model that simply takes the average of the word embedding vectors as the embedding of a language unit. For the language unit $u_{i}=\left\{w_{i, 1}, w_{i, 2}, \ldots w_{i, m}\right\}$, let $\boldsymbol{w} \boldsymbol{v}_{i, k}$ be the embedding vector of the word $w_{i, k}(k \in\{1,2, \ldots m\})$, the AVG-type embedding vector of $u_{i}$ can be calculate by the formula (2).

$$
\boldsymbol{u} \boldsymbol{v}_{i_{A V G}}=\frac{\sum_{k=1}^{m} \boldsymbol{w} \boldsymbol{v}_{i, k}}{m}
$$

The $\boldsymbol{w} \boldsymbol{v}_{i, k}$ is initialized by the publicly available 300 -dimensional GloVe vectors ${ }^{1}$. Stop words and words that have no embedding vector are removed from $u_{i}$ before embedding.

\section{- Smooth Inverse Frequency (SIF)}

SIF is another unsupervised model proposed in [49]. SIF model first computes the weighted average of the word embedding vectors as the initial embedding vector of each sentence, and then modifies these initial embedding vectors with the Principal Component Analysis to get the final embedding vector of each sentence. The experimental results in [49] show that this model improves about $10 \%$ to $30 \%$ than some baseline models, and can even beat some sophisticated supervised methods including RNN-based method and LSTM-based method.

\footnotetext{
${ }^{1}$ The package named as 'glove.840B.300d.zip' is downloaded from https://nlp.stanford.edu/projects/glove/.
} 
Let $D=\left\{u_{1}, u_{2}, \ldots, u_{t}\right\}$ be a source text that contains $t$ language units, $u_{i}=\left\{w_{i, 1}, w_{i, 2}, \ldots w_{i, m}\right\}$ be the $i^{\text {th }}$ language unit in $D$, and $\boldsymbol{w} \boldsymbol{v}_{i, k}$ be the embedding vector of word $w_{i, k}$. Let freq $\left(w_{i, k}\right)$ be the frequency of the word $w_{i, k}$, which is obtained by counting the word within a dataset of English Wikipedia ${ }^{2}$ in our implementation. Let $\boldsymbol{u} \boldsymbol{v}_{i_{\text {SIF }}}$ be the initial SIF-type embedding of $u_{i}$, and it is calculated by the formula (3). The constant parameter $\alpha$ in formula (3) is set as $10^{-3}$ both in [49] and in our implementation.

$$
\boldsymbol{u} \boldsymbol{v}_{i_{\text {SIF\# }}}=\frac{1}{m} \sum_{k=1}^{m} \frac{\alpha}{\operatorname{freq}\left(w_{i, k}\right)+\alpha} \boldsymbol{w} \boldsymbol{v}_{i, k}
$$

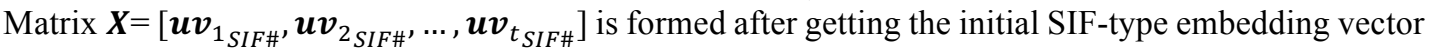
for each language unit. Then the Principal Component Analysis is conducted to assign the first singular vector of $\boldsymbol{X}$ to $\boldsymbol{y}$. Finally, the SIF-type embedding vectors for all the language units are calculated by formula (4).

$$
\left[u v_{1_{S I F}}, u v_{2_{S I F}}, \ldots, u v_{t_{S I F}}\right]=X-y y^{T} \cdot X
$$

\section{- Universal Sentence Encoder (USE)}

USE model is based on the deep averaging network where the embedding vectors for words and bigrams are averaged together and passed through a feed-forward deep neural network [50]. This model has been pre-trained and made freely available on Tensorflow $\mathrm{Hub}^{3}$. We can easily obtain the embedding vector of any language unit by loading and calling this open-source package.

After we get the embedding vectors of two language units $u_{i}$ and $u_{j}$, the similarity between them can be measured by formula (5), which is a variant of cosine similarity to ensure the similarity value to be positive. Note that $\boldsymbol{u} \boldsymbol{v}_{i}$ and $\boldsymbol{u} \boldsymbol{v}_{i}$ in formula (5) should be the same type of embedding vectors. We name the type of similarity between $u_{i}$ and $u_{j}$ as AVG-type if AVG model is used for calculating $\boldsymbol{u} \boldsymbol{v}_{i}$ and $\boldsymbol{u} \boldsymbol{v}_{j}$. The SIF-type similarity and the USE-type similarity can be got in a similar way.

$$
\operatorname{sim}\left(u_{i}, u_{j}\right)=\frac{1}{2} \times\left(\frac{\boldsymbol{u} \boldsymbol{v}_{i} \cdot \boldsymbol{u} \boldsymbol{v}_{j}}{\left|\boldsymbol{u} \boldsymbol{v}_{i}\right| \times\left|\boldsymbol{u} \boldsymbol{v}_{j}\right|}+1\right)
$$

\section{A.3 Synsets-based Similarity}

Many English words have more than one meaning. However, both the lexical-based similarity and the embedding-based similarity introduced above cannot detect the influence of polysemy on the similarity of two language units. The lexical-based similarity is based on the morphological matching of words without distinguishing the senses of words in different contexts. The embedding-based similarity is based on the word embedding. Although the word embedding tries to represent the sense of a word by the distances from this word to other words in a vector space, a single vector cannot represent different senses of a word at the same time. For example, if the embedding vector of 'apple' is close to the embedding vectors of 'company', 'products', 'computer' and 'smartphone', then the embedding vector of 'apple' should not be close to the embedding vectors of 'fruit', 'banana', 'red' and 'sweet'. Otherwise, the concept of 'electronic equipment manufacturer' and the concept of 'fruit' will be close in the embedding vector space, meaning that these two concepts are similar to each other.

The synsets-based similarity aims to not only reduce the similarity between two language units that contain polysemous words, such as "Mary likes apples" and "Mary likes the Apple Inc.", but also intensify the similarity between two language units that contain the synonyms, such as "this car uses a lot of oil" and "this automobile consumes a lot of petrol".

The synsets-based similarity between two language units is calculated by first assigning a sense to each word, then matching senses of words from the two language units according to the similarity of senses, and finally taking the average similarity of the matched sense pairs as the similarity between the two language units. Figure A-1 shows an example to calculate the synsets-based similarity between two sentences.

The synsets in WordNet are used as the senses of words [51]. Pywsd [52], a WordNet-based open-source word sense disambiguation package, is used to assign a specific synset to each word according to the adapted

\footnotetext{
2 The English Wikipedia dataset we used is downloaded from https://dumps.wikimedia.org/enwiki/20181101/.

${ }^{3}$ The instructions of USE model can be found at https://www.tensorflow.org/hub/modules/google/universal-sentence-encoder/1
} 
Lesk word sense disambiguation algorithm [53]. As for pairing words from two language units, we select two metrics to measure the similarity between two synsets.

- Lin

This metric is based on information content (IC) of synsets obtained from an extended corpus [54]. Let $s y n_{i}$ be the synset for word $w_{i}, s y n_{j}$ be the synset for word $w_{j}$, and $l c s\left(s y n_{i}, s y n_{j}\right)$ be the least common subsume of $s y n_{i}$ and $s y n_{j}$ in the WordNet taxonomy. The LIN metric calculates the similarity between $s y n_{i}$ and $s y n_{j}$ as shown in formula (6).

$$
\operatorname{LIN} \operatorname{sim}\left(\operatorname{syn}_{i}, \operatorname{syn}_{j}\right)=\frac{2 \times I C\left(\operatorname{lcs}\left(\operatorname{syn}_{i}, \operatorname{syn}_{j}\right)\right)}{I C\left(\operatorname{syn} n_{i}\right)+I C\left(\operatorname{syn}_{j}\right)}
$$

\section{- Wu and Palmer (WUP)}

As shown in formula (7), the WUP metric calculates the similarity between two synsets based on the depth of synsets in the WordNet taxonomy [55].

$$
W U P \operatorname{sim}\left(\operatorname{syn}_{i}, \operatorname{syn}_{j}\right)=\frac{2 \times \operatorname{depth}\left(\operatorname{lcs}\left(\operatorname{syn}_{i}, \operatorname{syn}_{j}\right)\right)}{\operatorname{depth}\left(\operatorname{syn}_{i}\right)+\operatorname{depth}\left(\operatorname{syn}_{j}\right)}
$$

Let $\left[\operatorname{syn}_{i, 1}, s y n_{i, 2}, \ldots, s y n_{i, m}\right]$ be the synsets list for $u_{i}$, and $\left[s y n_{j, 1}, s y n_{j, 2}, \ldots, s y n_{j, n}\right]$ be the synsets list for $u_{j}$. After getting the similarity for each pair like $\left(\operatorname{syn}_{i, x}, \operatorname{syn}_{j, y}\right)(1 \leq x \leq m, 1 \leq y \leq n)$ and storing them into a similarity matric $\operatorname{Syn} M$, we perform the Algorithm 1 to match synsets of $u_{i}$ and $u_{j}$. The similarity between $u_{i}$ and $u_{j}$ is the average similarity of the matched synsets. We name the type of the similarity between $u_{i}$ and $u_{j}$ LIN-type if the Lin metric is used to calculate the similarity for each synset pair, otherwise name WUP-type if the WUP metric is used.

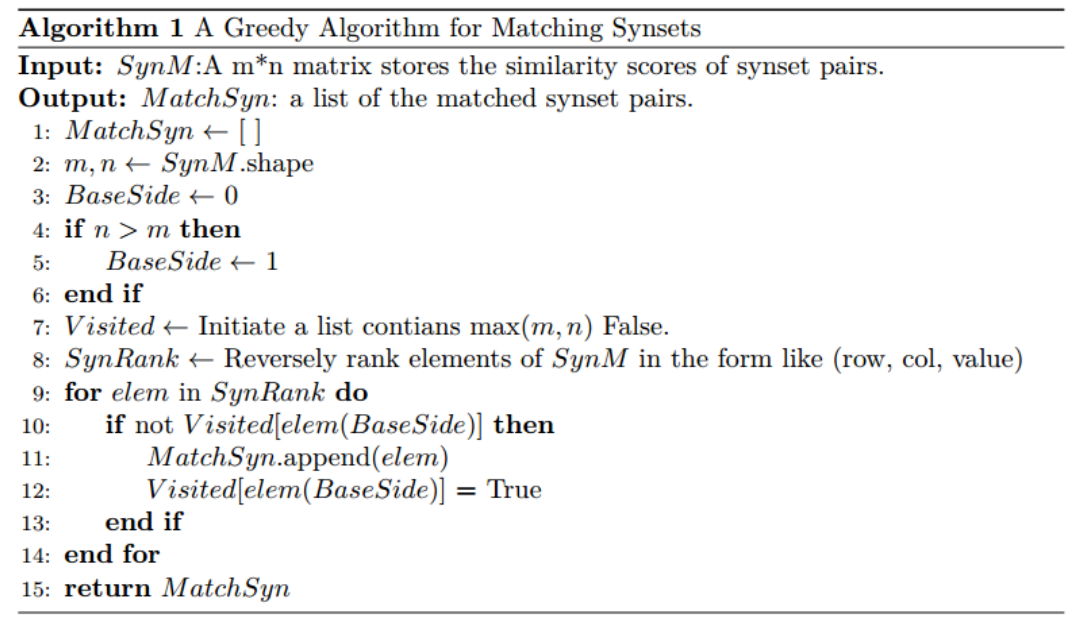

\section{Supplementary References}

[48] J. Pennington, R. Socher, C. Manning, Glove: Global vectors for word representation, in: Proceedings of the 2014 conference on Empirical Methods in Natural Language Processing (EMNLP), 2014, pp. 1532-1543.

[49] S. Arora, Y. Liang, T. Ma, A simple but tough-to-beat baseline for sentence embeddings, in: Proceedings of the International Conference on Learning Representations (ICLR), 2017.

[50] D. Cer, Y. Yang, S.-y. Kong, N. Hua, N. Limtiaco, R.S. John, N. Constant, M. Guajardo-Céspedes, K. Yuan, C. Tar, Y.-H. Sung, B. Strope, R. Kurzweil, Universal sentence encoder, arXiv: 1803.11175 [cs.CL], (2018).

[51] G.A. Miller, WordNet: A lexical database for English, Communications of the ACM, 38 (11) (1995) 39-41.

[52] L. Tan, Pywsd: Python implementations of Word Sense Disambiguation (WSD) technologies [software]. Retrieved from https://github.com/alvations/pywsd in GitHub, 2014. 
[53] S. Banerjee, T. Pedersen, An adapted lesk algorithm for word sense disambiguation using WordNet, in: Proceedings of international conference on intelligent text processing and computational linguistics, 2002, pp. 136-145.

[54] D. Lin, An information-theoretic definition of similarity, in: Proceedings of the $15^{\text {th }}$ international conference on machine learning, Morgan Kaufmann Publishers Inc., 1998, pp. 296-304.

[55] Z. Wu, M. Palmer, Verbs semantics and lexical selection, in: Proceedings of the $32^{\text {nd }}$ annual meeting on Association for Computational Linguistics (ACL), 1994, pp. 133-138.

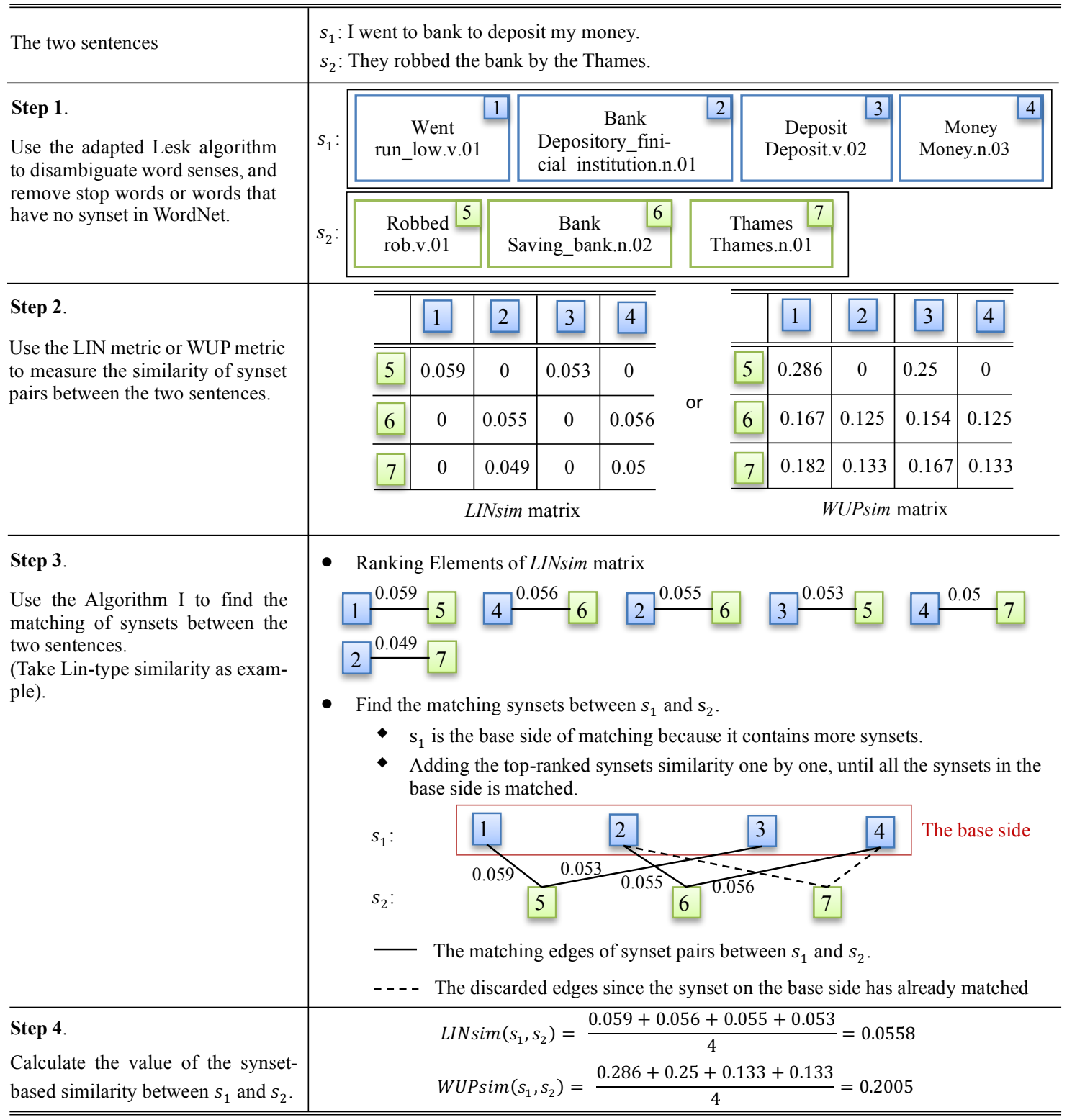

Figure A-1. The example of calculating the synsets-based similarity. 


\section{Appendix B. Seven Clustering Algorithms for Generating Groups}

Let $D$ be a source text that contains 4 paragraph $p_{1 \sim 4}$ and 16 sentences $s_{1 \sim 16}$. If we know $s_{2}-$ cause $\rightarrow s_{1}, s_{7}$ -cause $\rightarrow s_{8}$ and $s_{8}-$ cause $\rightarrow s_{9}$ and select the JCD-type similarity metric to calculate the weight of the similar-to links, then the first layer SLN for the source text $D$ is constructed as shown in Figure B-1.

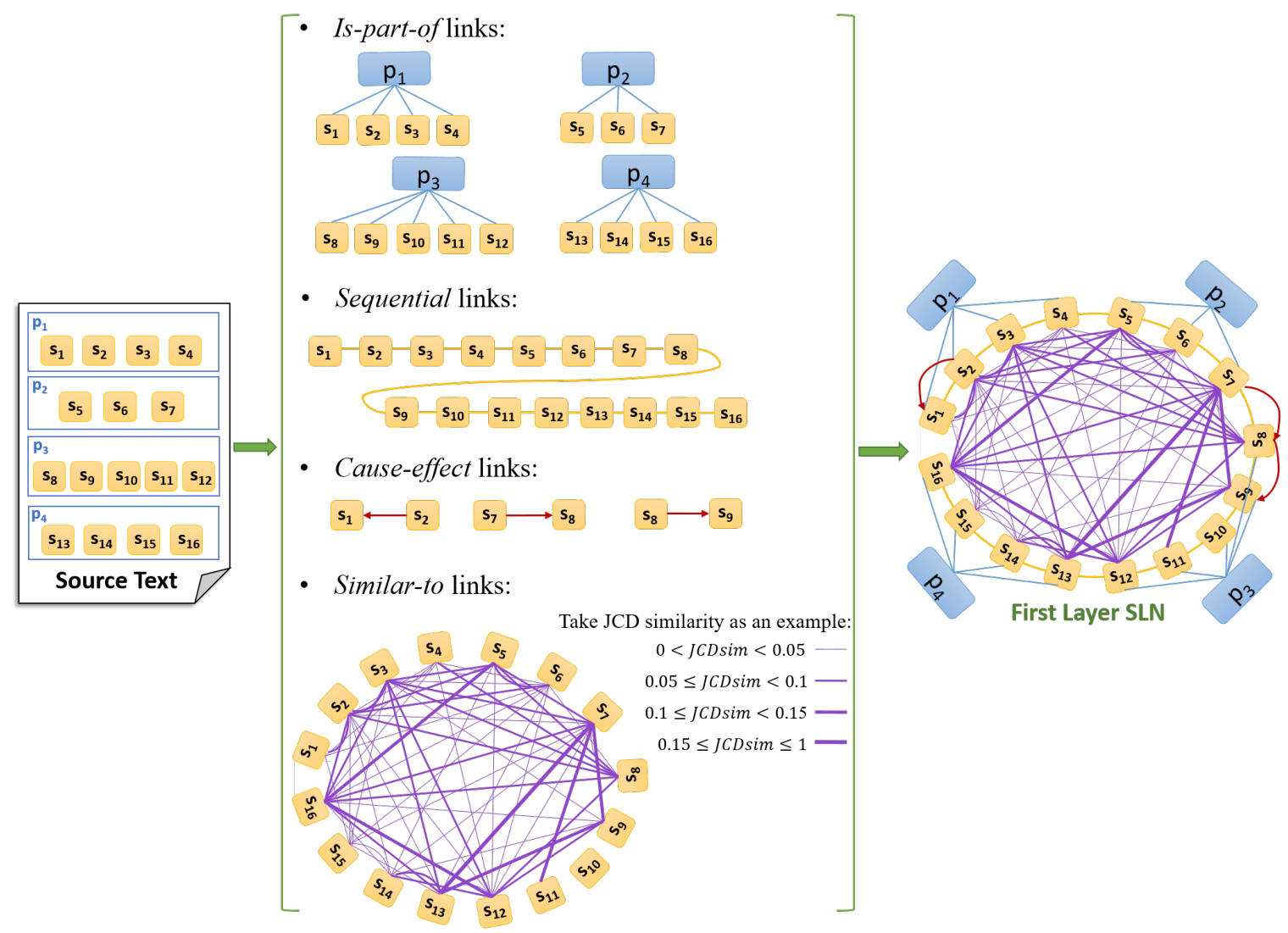

Fig B-1. An example of constructing the first layer SLN for a source text.

Seven clustering algorithms are designed for generating groups according to the combinations of the ispart-of link, sequential link, similar-to link, and cause-effect link:

- Algorithm 1: Seq+pb/npb (win_size $\in N^{+}$)

As shown in Figure B-1, this clustering algorithm slides a window along with the sequential links and clusters sentences within the window as a group. The parameter ' win_size' is a positive integer specifying the number of sentences covered by the sliding window. The ' $+p b$ ' represents the 'paragraph-bounded' mode, in which the is-part-of link is used to check the subordination between sentences and paragraphs to ensure that the sentences within a group belong to the same paragraph. In contrast, the ' $+n p b$ ' represents the 'not paragraph-bounded' mode, in which sentences from two consecutive paragraphs can be clustered into the same group. Either the ' $+p b$ ' mode or the ' $+n p b$ ' mode should be chosen when using this algorithm.

When composing a summary with top-ranked groups, we just put the first few sentences of the last extracted group into the summary if the group contains too many sentences to be completely put into the summary. Therefore, the order of the sentences within a group affects the sentences contained in the generated summary. However, ordering the sentences within a group differently from the source text disrupts the semantic link between sentences, so we arrange the sentences of each group according to the sequential link if it is used to generate groups. 


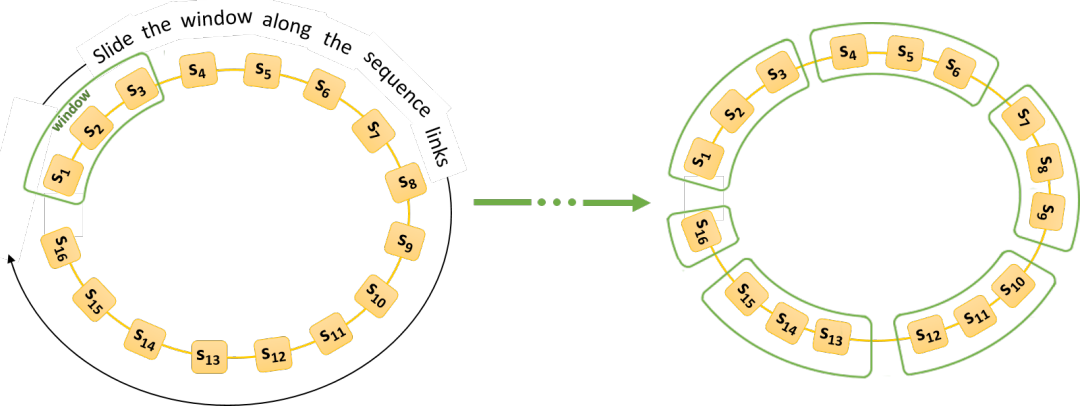

(a) $\operatorname{Seq}+n p b($ win_size $=3)$

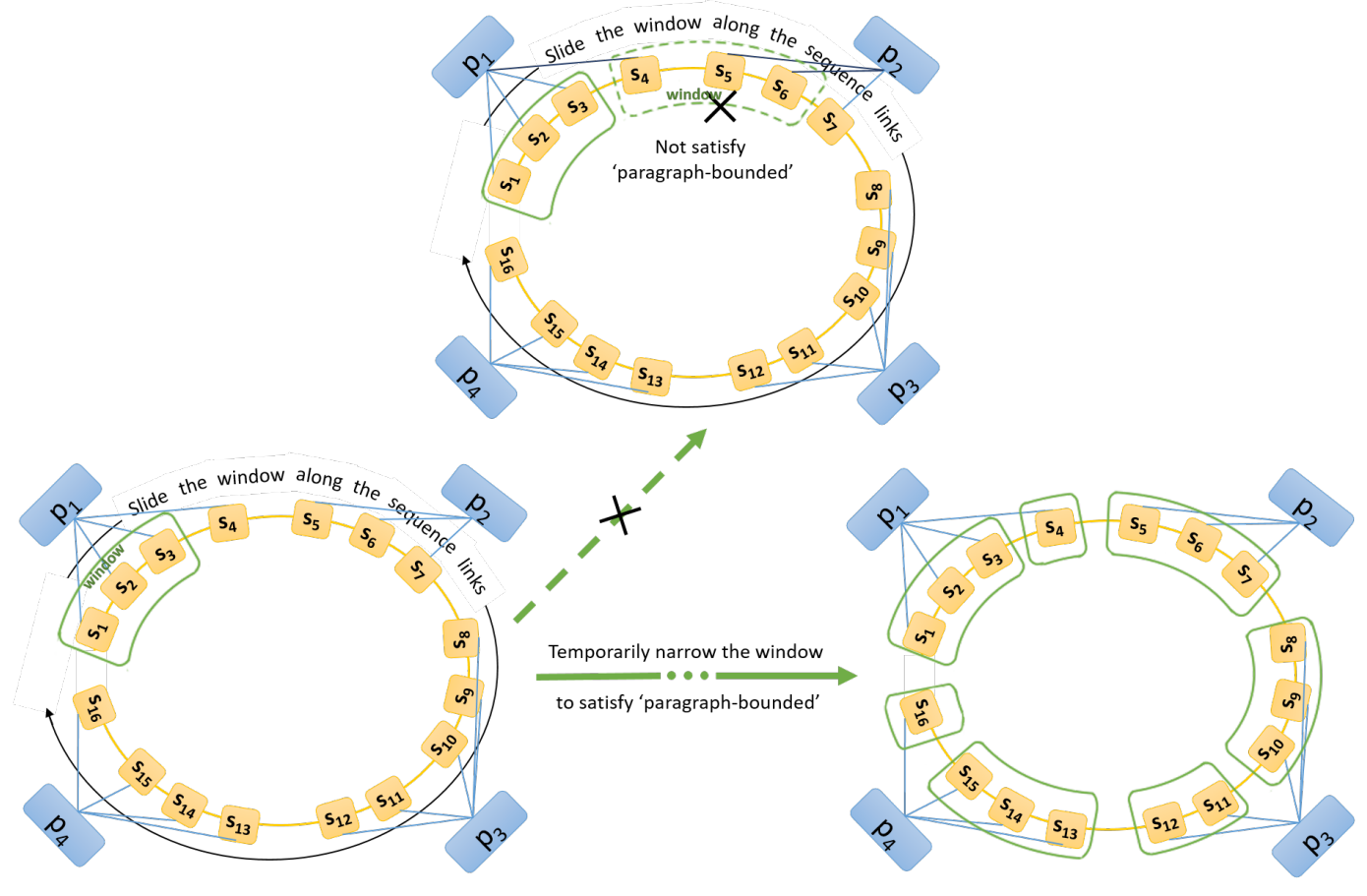

(b) $S e q+p b\left(w i n \_s i z e=3\right)$

Figure B-2. The clustering process of the $\operatorname{Seq}+p b / n p b($ win_size $=3)$ algorithm.

\section{- Algorithm 2: SimSIZE (sim_type, size $\in N^{+}$)}

This algorithm clusters sentences into groups of fixed size according to the similar-to links between sentences. The parameter 'sim_type' takes one in $\{\mathrm{JCD}$, AVG, SIF, USE, LIN, WUP $\}$, referring to the type of the similar-to links used to cluster sentences. The parameter 'size' specifies the maximum number of sentences within a group.

As shown in Figure B-3, the clustering process of the SimSIZE algorithm is iterative. For each iteration, the algorithm first sorts all similar-to links in descending order, next checks the similar-to link in turn until finding the first similar-to link whose nodes contain no more than 'size' sentences, then merges the two nodes of this similar-to link to form a new node, and finally calculates the average similar-to links to connect the newly formed node with other nodes. Perform the above iteration until no similar-to link can be merged, at which point each node is a group.

In order to determine the order of sentences within each group, we add an attribute named 'tightness' to each node during the clustering process. The tightness of a node containing only one sentence is set zero, and the tightness of a node containing more than one sentence is equal to the sum of the weights of the similar-to links that are used to form this node divided by the number of sentences contained in this node. When merging two nodes to form a new node, we put the sentences of the high tightness node before the sentences of the low tightness node. 

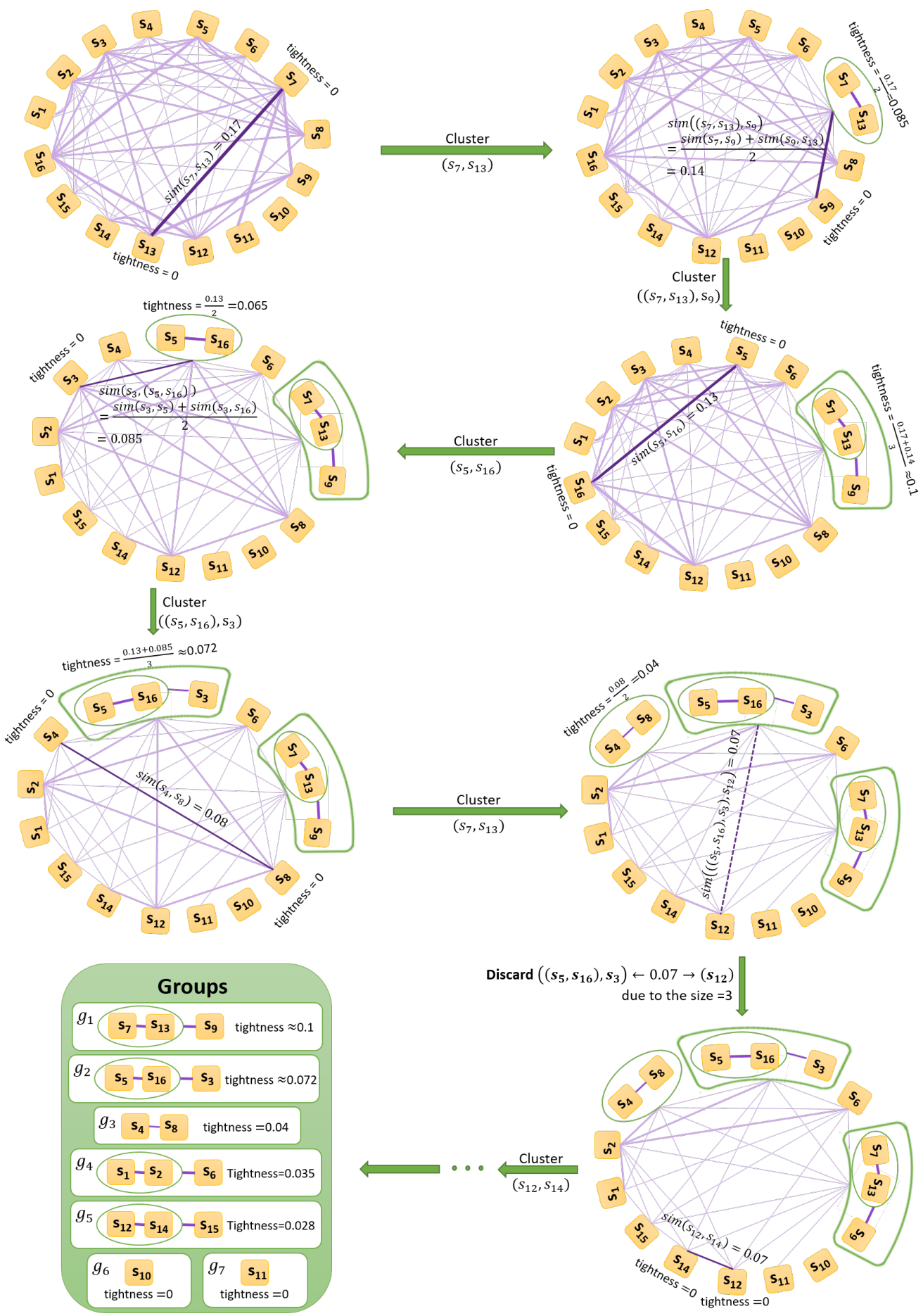

Figure B-3. The clustering process of the $S e q+p b / n p b($ win_size $=3)$ algorithm.

\section{- Algorithm 3: SimTHR (sim_type,thr $\in R^{+}$)}

The SimTHR algorithm is similar to the SimSIZE algorithm, except that it uses a threshold called 'thr' to restrict the weight of the similar-to link that is selected to form the new node in each iteration, without limiting the number of sentences in the newly formed node. The iteration will be stopped when there is no similar-to link whose weight is larger than the threshold 'thr', at which point each node is a group. Figure B-4 shows the clustering process of the SimTHR algorithm. 

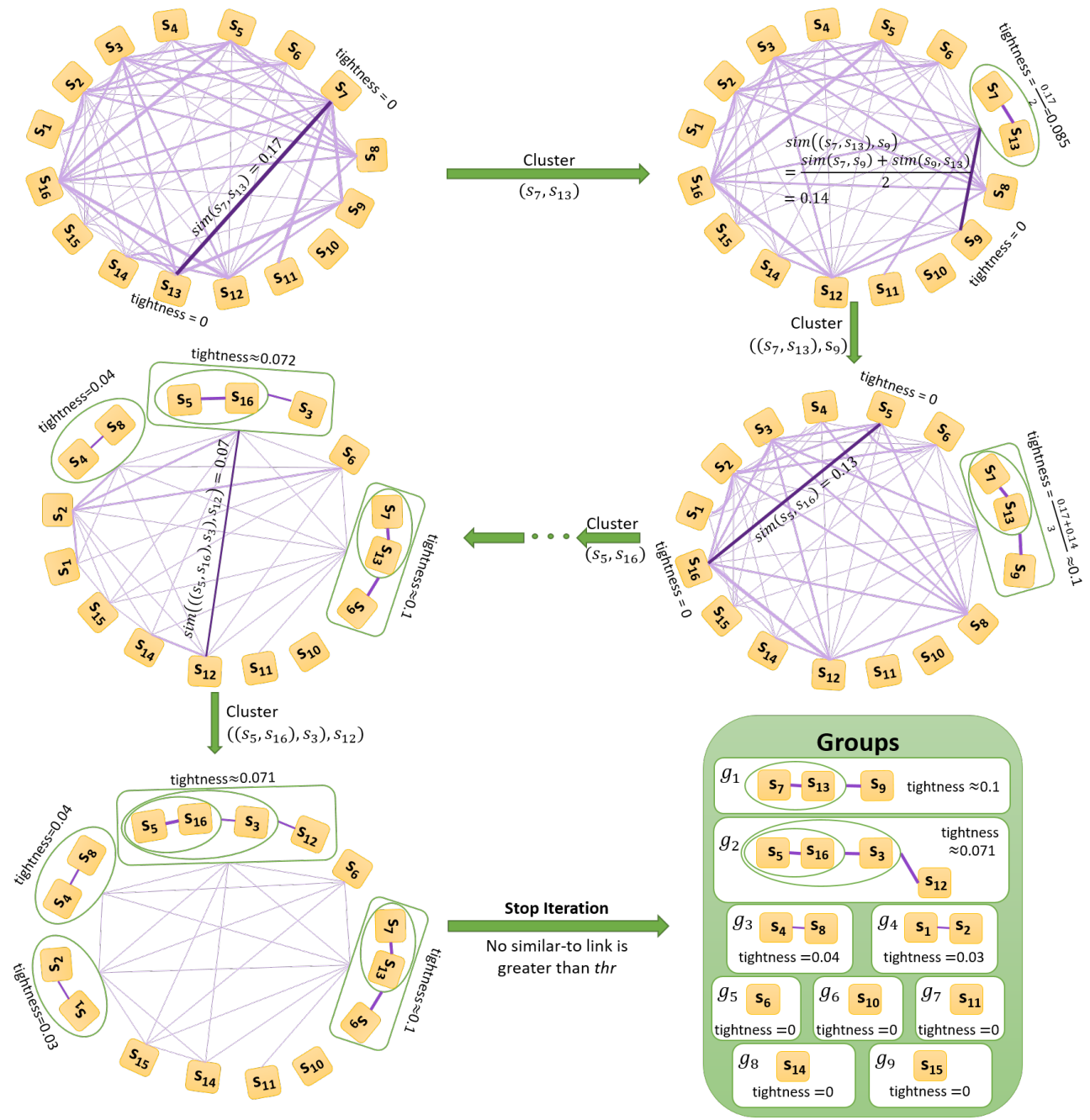

Figure B-4. The clustering process of the SimTHR(JCD, thr $=0.05)$ algorithm.

\section{- Algorithm 4: $C E+p b / n p b$}

This algorithm clusters sentences that are connected by cause-effect links into a group, and views each sentence that is not linked by a cause-effect link as a group. In the ' $+n p b$ ' mode all the cause-effect links are used to cluster sentences into groups, while in the ' $+p b$ ' mode the cause-effect links whose sentences do not belong to the same paragraph are discarded before clustering. Figure B-5 shows examples of generating groups with the $C E+p b / n p b$ algorithm.

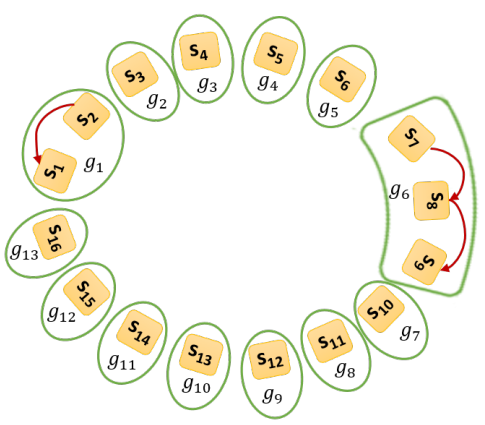

(a) $C E+n p b$

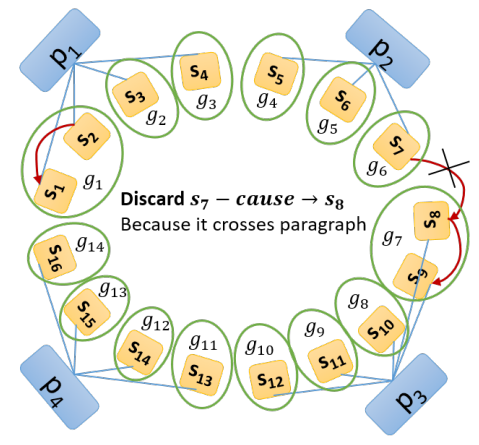

(b) $C E+p b$

Figure B-5. The clustering process of the $C E+p b / n p b$ algorithm. 


\section{- Algorithm 5: CESim(sim_type,thr $\in R^{+}$)}

As shown in Figure B-6, the CESim algorithm first performs the $C E+n p b$ algorithm to cluster sentences into temporary groups, then connects these temporary groups by averaging the similar-to links between sentences, and then performs the SimTHR algorithm to cluster temporary groups into the final groups. The ' $+n p b$ ' mode is selected for the $C E$ algorithm because the paragraph-bounded restriction is useless in the clustering process of the SimTHR algorithm.

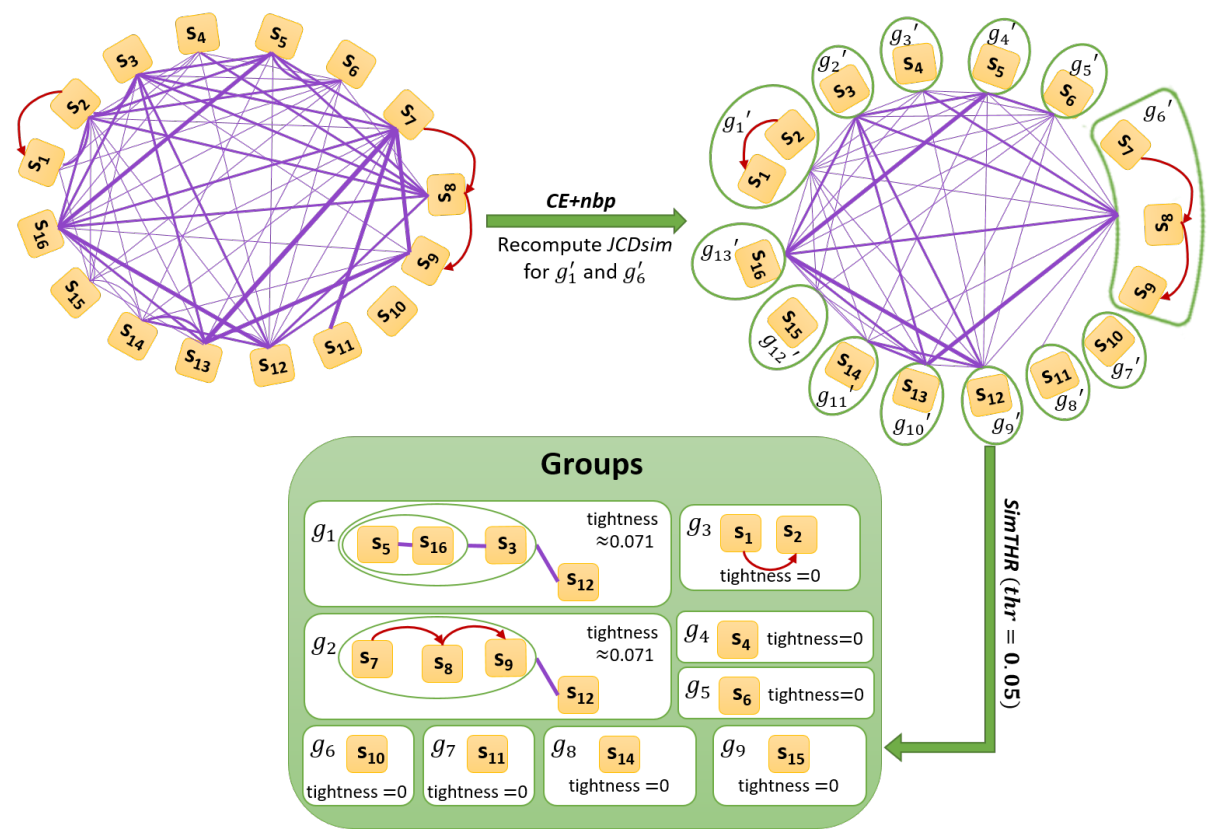

Figure B-6. The clustering process of the $C E \operatorname{Sim}(J C D, t h r=0.05)$ algorithm.

\section{- Algorithm 6: SeqSim+pb/npb(sim_type,thr $\left.\in R^{+}\right)$}

Figure B-7 and Figure B-8 respectively show the clustering process of the SeqSim algorithm in the modes of ' $+n p b$ ' and ' $+p b$ '. At first, the algorithm initializes the first sentence of a source text as a group. Then, from the second sentence, the algorithm checks each sentence along the sequential link to determine whether the sentence should be added to the previous group or should be initialized as a new group according to the similar-to links between this sentence and the sentences in the previous group. For example, let $s_{c+1}$ be the sentence being checked and $g_{b}=\left(s_{c-2}, s_{c-1}, s_{c}\right)$ be the group before $s_{c+1}$. If the weight of a similar-to link between $s_{c+1}$ and any sentence in $g_{b}$ is greater than the threshold 'thr', then $s_{c+1}$ should be appended to $g_{b}$. If not, the algorithm initializes $s_{c+1}$ as a new group $g_{b+1}=\left(s_{c+1}\right)$.

The ' $+p b$ ' mode requires that the sentences within a group belong to the same paragraph, so the SeqSim $+p b$ algorithm initializes the first sentence of each paragraph as a new group regardless of the weights of the similar-to links.

\section{- Algorithm 7: CESeqSim+pb/npb(sim_type,thr $\left.\in R^{+}\right)$}

The CESeqSim algorithm is the combination of the CE algorithm and the SeqSim algorithm. As shown in Figure B-9, it first performs the $C E$ algorithm to cluster sentences into temporary groups while remaining the sequential links and the is-part-of links on these temporary groups, then connects these temporary groups by the average similar-to links, and finally performs the SeqSim algorithm to cluster the temporary groups into the final groups. Note that the mode of the paragraph-bounded restriction must be consistent in the clustering processes of both the $C E$ algorithm and the SeqSim algorithm. 

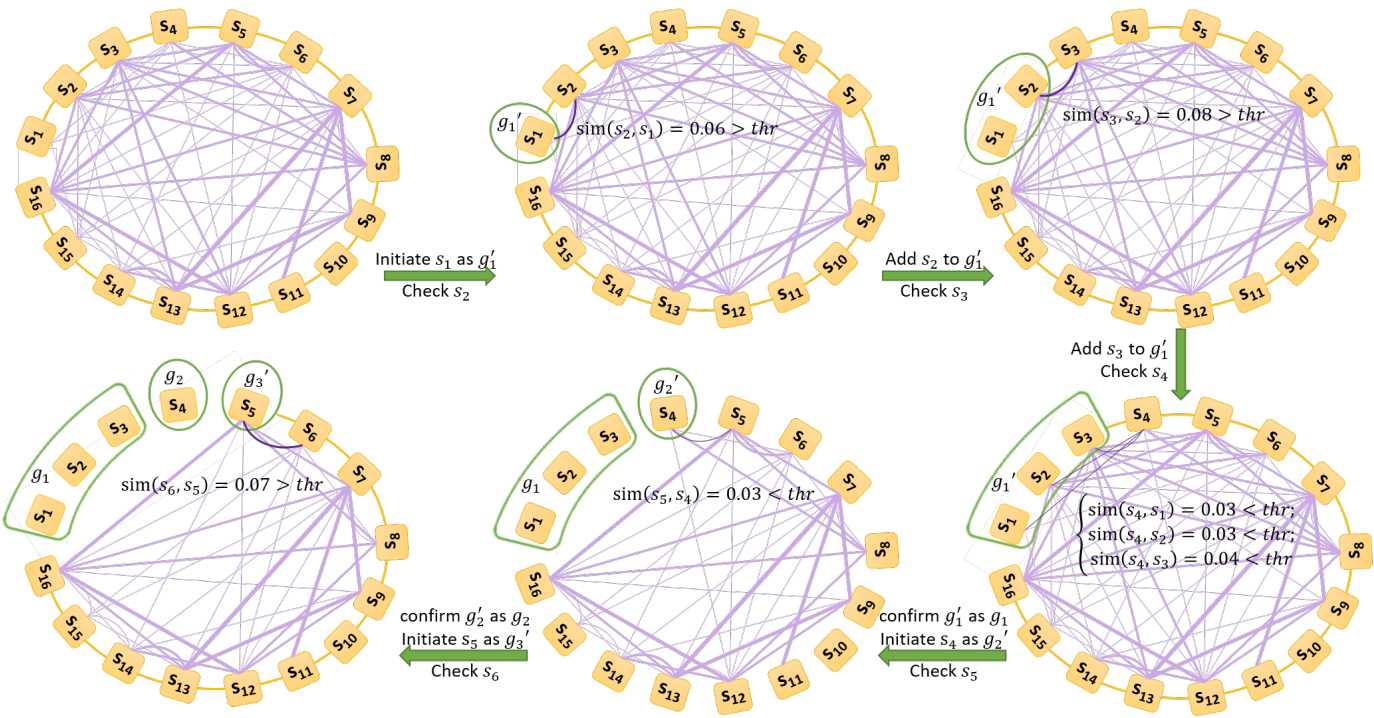

Add $s_{6}$ to $g_{3}^{\prime}$
Check $s_{7}$
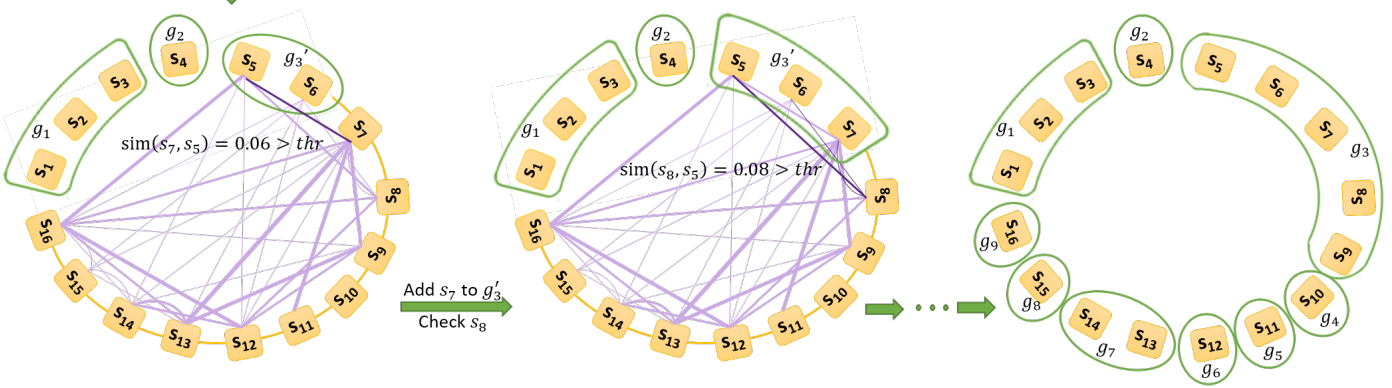

Figure B-7. The clustering process of the SeqSim $+n p b(J C D, t h r=0.05)$ algorithm.
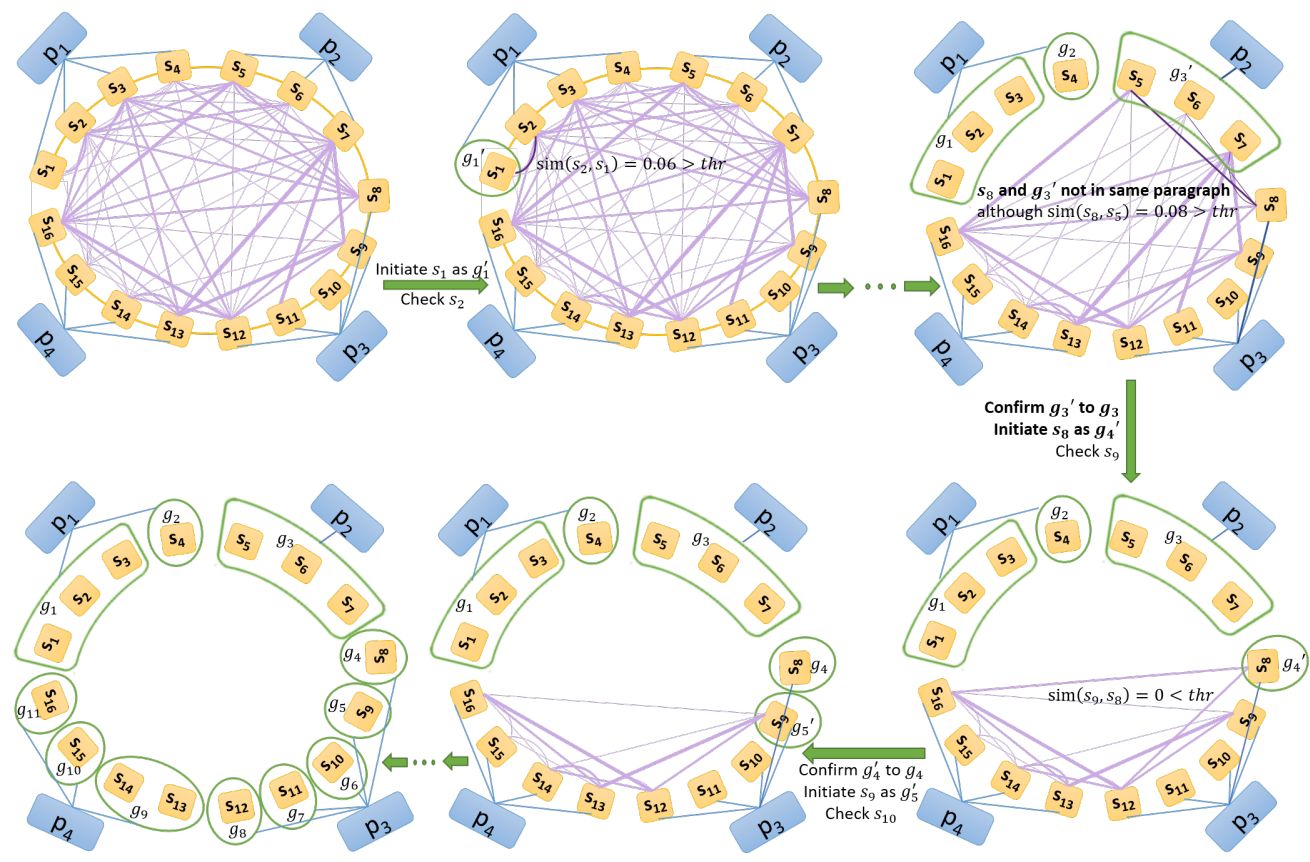

Figure B-8. The clustering process of the SeqSim $+p b(J C D, t h r=0.05)$ algorithm. 


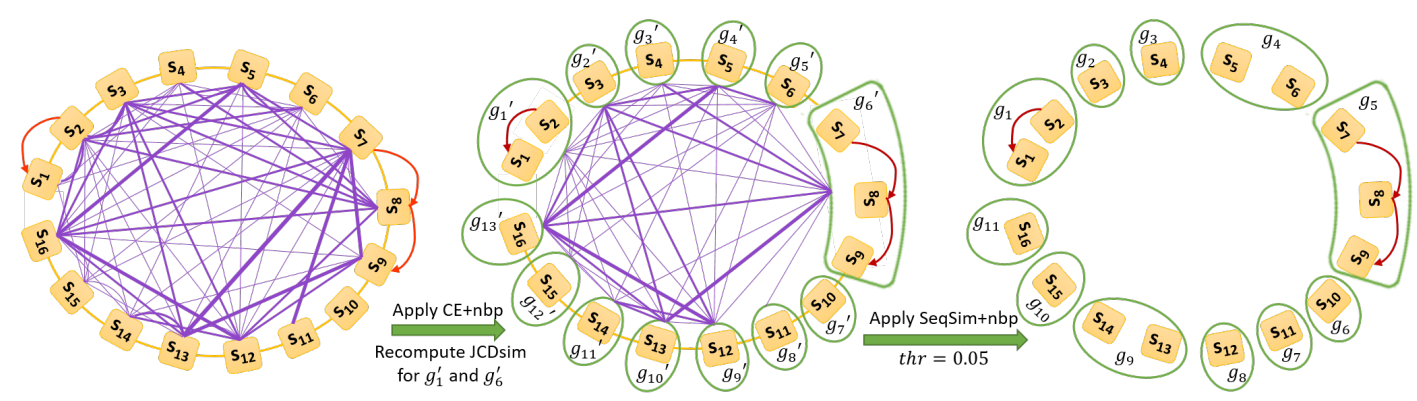

(a) CESeqSim $+n p b(J C D, t h r=0.05)$

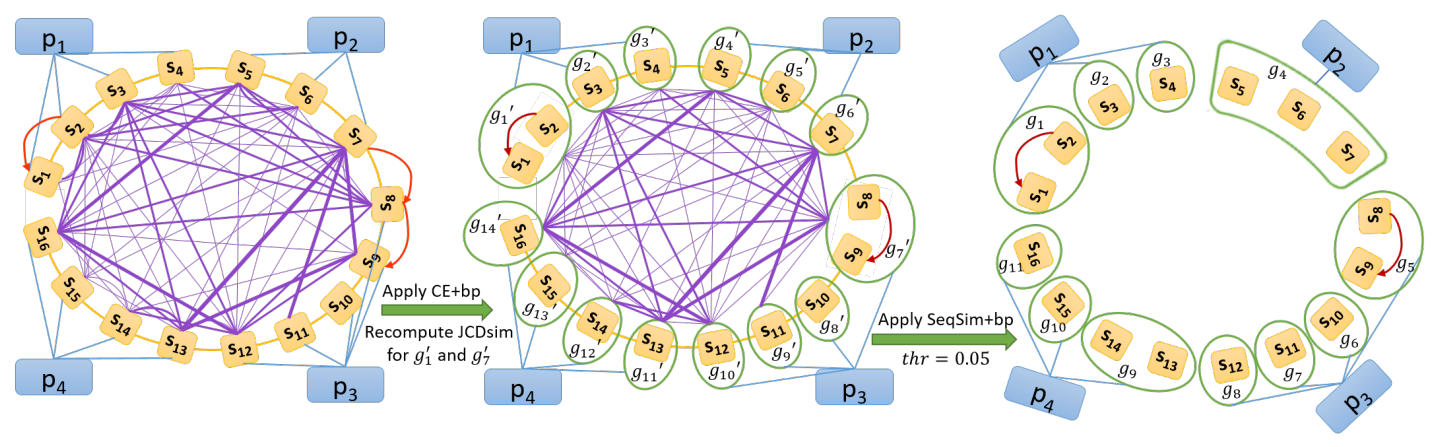

(b) CESeqSim $+p b(J C D$, thr $=0.05)$

Figure B-9. The clustering process of the CESeqSim $(J C D, t h r=0.05)$ algorithm. 


\section{Appendix C. The Supplemental Results of the Four Metrics}

\section{C.1 The Preliminary Comparison}

We first use the SeqSim $+p b(J C D, 0.06)$ algorithm to generate groups and use the JCD-type sim-SLN to rank sentences, groups or paragraphs. Figure C-1 shows the values of the four metrics on each sub-dataset when using the Conclusion or the Introduction of each paper as the standard summaries.

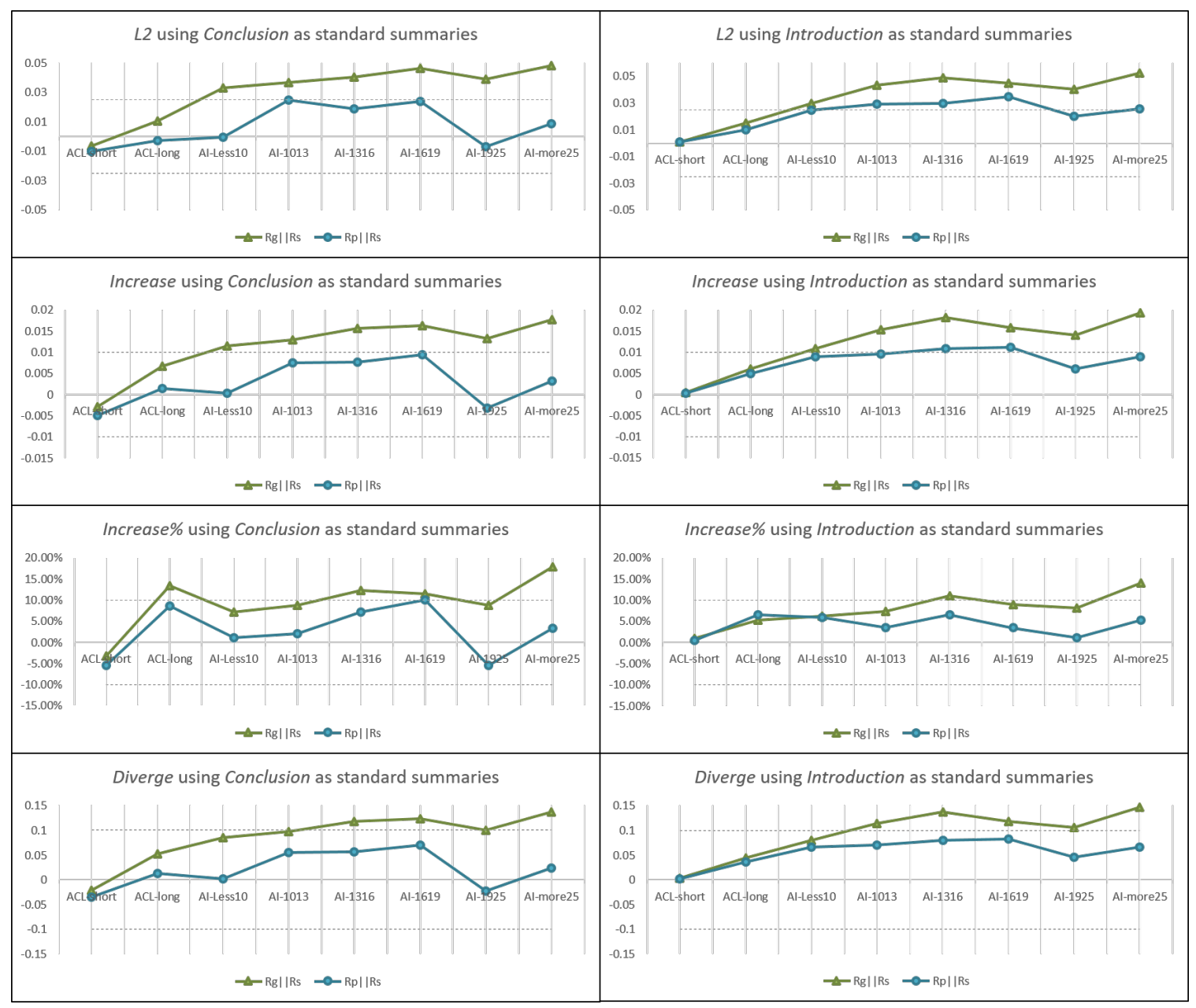

Figure C-1. The four metrics on each sub-dataset when using SeqSim $+p b(J C D, 0.06)$ for generating groups and using JCD-type simSLNs for ranking.

\section{C.2 The Further Comparison by Changing Types of sim-SLN}

We still use the SeqSim $+p b(J C D, 0.06)$ algorithm to generate groups, but change the types of sim-SLN for ranking language units. Table $\mathrm{C}-1$ to Table $\mathrm{C}-4$ separately shows the results of the four metrics on the $A C L$ all dataset and the $A I$-all dataset when changing the types of sim-SLN and the kinds of standard summaries. Table C-5 to Table C-8 separately shows the results of the four metrics on each sub-dataset when changing the types of sim-SLN and the kinds of standard summaries.

Table C-1. The $L 2$ metric on the $A C L$-all and $A I$-all datasets when using different sim-SLNs and different standard summary.

\begin{tabular}{c|c|c}
\hline \hline$L 2$ metric & The $A C L$-all dataset & The AI-all dataset \\
\hline
\end{tabular}




\begin{tabular}{c|l|l|l|l|l|l|l}
\hline \multicolumn{2}{c|}{} & Abstract & Conclusion & Introduction & Abstract & Conclusion & Introduction \\
\hline \hline \multirow{2}{*}{ JCD-type sim-SLNs } & $R_{g} \| R_{s}$ & 0.0057 & 0.0024 & 0.0076 & 0.0348 & 0.0409 & 0.0433 \\
\cline { 2 - 8 } & $R_{p} \| R_{s}$ & -0.0084 & -0.0063 & 0.0052 & 0.0061 & 0.0115 & 0.0275 \\
\hline \multirow{2}{*}{ AVG-type sim-SLNs } & $R_{g} \| R_{s}$ & 0.0083 & 0.0035 & -0.0085 & 0.0018 & 0.0025 & -0.0205 \\
\cline { 2 - 8 } & $R_{p} \| R_{s}$ & -0.0175 & -0.0115 & -0.0132 & 0.0112 & 0.0100 & -0.0019 \\
\hline \multirow{2}{*}{ SIF-type sim-SLNs } & $R_{g} \| R_{s}$ & 0.0524 & 0.0499 & 0.0415 & -0.0033 & -0.0047 & -0.0263 \\
\cline { 2 - 8 } & $R_{p} \| R_{s}$ & 0.0607 & 0.0685 & 0.0589 & -0.0156 & -0.0079 & -0.0250 \\
\hline \multirow{2}{*}{ GSE-type sim-SLNs } & $R_{g} \| R_{s}$ & -0.0042 & 0.0018 & -0.0105 & 0.0155 & 0.0247 & 0.0348 \\
\cline { 2 - 8 } & $R_{p} \| R_{s}$ & -0.0124 & 0.0004 & -0.0126 & 0.0138 & 0.0188 & 0.0310 \\
\hline \multirow{2}{*}{ LIN-type sim-SLNs } & $R_{g} \| R_{s}$ & -0.0199 & -0.0045 & 0.0014 & 0.0201 & 0.0178 & 0.0109 \\
\cline { 2 - 8 } & $R_{p} \| R_{s}$ & -0.0102 & 0.0041 & -0.0009 & 0.0211 & 0.0199 & 0.0191 \\
\hline \multirow{2}{*}{ WUP-type sim-SLNs } & $R_{g} \| R_{s}$ & 0.0056 & 0.0208 & 0.0125 & 0.0272 & 0.0275 & 0.0282 \\
\cline { 2 - 7 } & $R_{p} \| R_{s}$ & 0.0132 & 0.0318 & 0.0189 & 0.0375 & 0.0405 & 0.0427 \\
\hline
\end{tabular}

Table C-2. The Increase metric on the $A C L$-all and AI-all datasets when using different sim-SLNs and different standard summary.

\begin{tabular}{l|l|l|l|l|l|l|l}
\hline \hline \multirow{2}{*}{\begin{tabular}{l} 
Increase metric \\
\cline { 3 - 8 }
\end{tabular}} & \multicolumn{3}{c|}{ The ACL-all dataset } & \multicolumn{3}{c}{ The AI-all dataset } \\
\cline { 3 - 8 } & Abstract & Conclusion & Introduction & Abstract & Conclusion & Introduction \\
\hline \hline \multirow{2}{*}{ JCD-type sim-SLNs } & $R_{g} \| R_{s}$ & 0.0031 & 0.0021 & 0.0031 & 0.0130 & 0.0147 & 0.0156 \\
\cline { 2 - 8 } & $R_{p} \| R_{s}$ & -0.0007 & -0.0015 & 0.0025 & 0.0033 & 0.0042 & 0.0093 \\
\hline \multirow{2}{*}{ AVG-type sim-SLNs } & $R_{g} \| R_{s}$ & 0.0030 & 0.0031 & -0.0025 & 0.0006 & 0.0008 & -0.0082 \\
\cline { 2 - 8 } & $R_{p} \| R_{s}$ & -0.0073 & -0.0027 & -0.0047 & 0.0033 & 0.0025 & -0.0022 \\
\hline \multirow{2}{*}{ SIF-type sim-SLNs } & $R_{g} \| R_{s}$ & 0.0207 & 0.0195 & 0.0155 & -0.0014 & -0.0012 & -0.0081 \\
\cline { 2 - 8 } & $R_{p} \| R_{s}$ & 0.0236 & 0.0246 & 0.0223 & -0.0080 & -0.0046 & -0.0102 \\
\hline \multirow{2}{*}{ GSE-type sim-SLNs } & $R_{g} \| R_{s}$ & -0.0001 & 0.0032 & -0.0033 & 0.0053 & 0.0086 & 0.0125 \\
\cline { 2 - 8 } & $R_{p} \| R_{s}$ & -0.0033 & 0.0028 & -0.0038 & 0.0055 & 0.0062 & 0.0111 \\
\hline \multirow{2}{*}{ LIN-type sim-SLNs } & $R_{g} \| R_{s}$ & -0.0072 & 0.0002 & 0.0012 & 0.0061 & 0.0055 & 0.0028 \\
\cline { 2 - 8 } & $R_{p} \| R_{s}$ & -0.0003 & 0.0052 & 0.0012 & 0.0063 & 0.0059 & 0.0055 \\
\hline \multirow{2}{*}{ WUP-type sim-SLNs } & $R_{g} \| R_{s}$ & 0.0040 & 0.0096 & 0.0066 & 0.0086 & 0.0083 & 0.0092 \\
\cline { 2 - 7 } & $R_{p} \| R_{s}$ & 0.0073 & 0.0147 & 0.0099 & 0.0119 & 0.0123 & 0.0142 \\
\hline
\end{tabular}

Table C-3. The Increase\% metric on the $A C L$-all and AI-all datasets when using different sim-SLNs and different standard summary.

\begin{tabular}{l|l|l|l|l|l|l|l}
\hline \hline \multicolumn{2}{c}{ Increase\% metric } & \multicolumn{3}{c|}{ The ACL-all dataset } & \multicolumn{3}{c}{ The AI-all dataset } \\
\cline { 3 - 8 } \multicolumn{2}{c|}{} & Abstract & Conclusion & Introduction & Abstract & Conclusion & Introduction \\
\hline \hline \multirow{2}{*}{ JCD-type sim-SLNs } & $R_{g} \| R_{s}$ & 0.0475 & 0.0557 & 0.0312 & 0.1671 & 0.1124 & 0.0921 \\
\cline { 2 - 8 } & $R_{p} \| R_{s}$ & 0.0546 & 0.0204 & 0.0359 & 0.0924 & 0.0335 & 0.0441 \\
\hline \multirow{2}{*}{ AVG-type sim-SLNs } & $R_{g} \| R_{s}$ & 0.0236 & 0.0871 & 0.0066 & 0.0325 & 0.0124 & -0.0560 \\
\cline { 2 - 8 } & $R_{p} \| R_{s}$ & -0.0969 & 0.0283 & -0.0182 & 0.0368 & -0.0015 & -0.0383 \\
\hline \multirow{2}{*}{ SIF-type sim-SLNs } & $R_{g} \| R_{s}$ & 0.4885 & 0.3132 & 0.1313 & -0.0169 & 0.0009 & -0.0126 \\
\cline { 2 - 8 } & $R_{p} \| R_{s}$ & 0.5103 & 0.3184 & 0.1950 & -0.1615 & -0.0745 & -0.0672 \\
\hline \multirow{2}{*}{ GSE-type sim-SLNs } & $R_{g} \| R_{s}$ & 0.0557 & 0.1309 & -0.0090 & 0.0631 & 0.0548 & 0.0725 \\
\cline { 2 - 8 } & $R_{p} \| R_{s}$ & 0.0071 & 0.1272 & -0.0075 & 0.1432 & 0.0374 & 0.0651 \\
\hline \multirow{2}{*}{ LIN-type sim-SLNs } & $R_{g} \| R_{s}$ & -0.0553 & 0.0566 & 0.0286 & 0.0759 & 0.0319 & -0.0137 \\
\cline { 2 - 8 } & $R_{p} \| R_{s}$ & 0.1073 & 0.1575 & 0.0456 & 0.0332 & 0.0193 & 0.0018 \\
\hline WUP-type sim-SLNs & $R_{g} \| R_{s}$ & 0.1242 & 0.1339 & 0.0922 & 0.0878 & 0.0345 & 0.0408 \\
\hline
\end{tabular}




\begin{tabular}{|l|l|l|l|l|l|l}
\hline$R_{p} \| R_{s}$ & 0.1686 & 0.2017 & 0.1326 & 0.1229 & 0.0491 & 0.0744 \\
\hline
\end{tabular}

Table C-4. The Diverge metric on the ACL-all and AI-all datasets when using different sim-SLNs and different standard summary.

\begin{tabular}{l|l|l|l|l|l|l|l}
\hline \hline \multirow{2}{*}{\multicolumn{2}{c|}{ Diverge metric }} & \multicolumn{3}{c|}{ The ACL-all dataset } & \multicolumn{3}{c}{ The AI-all dataset } \\
\cline { 3 - 8 } \multicolumn{2}{l|}{} & Abstract & Conclusion & Introduction & Abstract & Conclusion & Introduction \\
\hline \hline \multirow{2}{*}{ JCD-type sim-SLNs } & $R_{g} \| R_{s}$ & 0.0232 & 0.0160 & 0.0230 & 0.0989 & 0.1109 & 0.1170 \\
\cline { 2 - 8 } & $R_{p} \| R_{s}$ & -0.0040 & -0.0106 & 0.0180 & 0.0247 & 0.0306 & 0.0685 \\
\hline \multirow{2}{*}{ AVG-type sim-SLNs } & $R_{g} \| R_{s}$ & 0.0222 & 0.0235 & -0.0179 & 0.0050 & 0.0060 & -0.0582 \\
\cline { 2 - 8 } & $R_{p} \| R_{s}$ & -0.0502 & -0.0191 & -0.0335 & 0.0245 & 0.0187 & -0.0155 \\
\hline \multirow{2}{*}{ SIF-type sim-SLNs } & $R_{g} \| R_{s}$ & 0.1671 & 0.1536 & 0.1170 & -0.0097 & -0.0086 & -0.0570 \\
\cline { 2 - 8 } & $R_{p} \| R_{s}$ & 0.1915 & 0.1947 & 0.1706 & -0.0538 & -0.0317 & -0.0714 \\
\hline \multirow{2}{*}{ GSE-type sim-SLNs } & $R_{g} \| R_{s}$ & -0.0001 & 0.0256 & -0.0233 & 0.0394 & 0.0633 & 0.0932 \\
\cline { 2 - 8 } & $R_{p} \| R_{s}$ & -0.0236 & 0.0220 & -0.0271 & 0.0413 & 0.0453 & 0.0820 \\
\hline \multirow{2}{*}{ LIN-type sim-SLNs } & $R_{g} \| R_{s}$ & -0.0506 & 0.0025 & 0.0092 & 0.0454 & 0.0408 & 0.0202 \\
\cline { 2 - 8 } & $R_{p} \| R_{s}$ & 0.0004 & 0.0415 & 0.0089 & 0.0470 & 0.0437 & 0.0402 \\
\hline \multirow{2}{*}{ WUP-type sim-SLNs } & $R_{g} \| R_{s}$ & 0.0309 & 0.0726 & 0.0496 & 0.0648 & 0.0622 & 0.0683 \\
\cline { 2 - 7 } & $R_{p} \| R_{s}$ & 0.0563 & 0.1134 & 0.0747 & 0.0912 & 0.0932 & 0.1071 \\
\hline
\end{tabular}

Table C-5. The $L 2$ metric on each sub-dataset when using different sim-SLNs and different standard summary.

\begin{tabular}{|c|c|c|c|c|c|c|c|c|c|c|c|c|c|}
\hline \multicolumn{2}{|c|}{ L2 metric } & \multicolumn{2}{|c|}{$\begin{array}{l}\text { JCD-type sim- } \\
\text { SLNs }\end{array}$} & \multicolumn{2}{|c|}{$\begin{array}{l}\text { AVG-type sim- } \\
\text { SLNs }\end{array}$} & \multicolumn{2}{|c|}{$\begin{array}{l}\text { SIF-type sim- } \\
\text { SLNs }\end{array}$} & \multicolumn{2}{|c|}{$\begin{array}{l}\text { GSE-type sim- } \\
\text { SLNs }\end{array}$} & \multicolumn{2}{|c|}{$\begin{array}{l}\text { LIN-type sim- } \\
\text { SLNs }\end{array}$} & \multicolumn{2}{|c|}{$\begin{array}{l}\text { WUP-type sim- } \\
\text { SLNs }\end{array}$} \\
\hline & & $R_{g} \| R_{S}$ & $R_{p} \| R_{s}$ & $R_{g} \| R_{s}$ & $R_{p} \| R_{s}$ & $R_{g} \| R_{s}$ & $R_{p} \| R_{s}$ & $R_{g} \| R_{s}$ & $R_{p} \| R_{s}$ & $R_{g} \| R_{s}$ & $R_{p} \| R_{s}$ & $R_{g} \| R_{s}$ & $R_{p} \| R_{s}$ \\
\hline \multirow{3}{*}{$A C L$-short } & Abs. & 0.007 & 0.005 & 0.020 & -0.004 & 0.023 & 0.036 & 0.003 & 0.002 & -0.029 & -0.010 & 0.002 & 0.021 \\
\hline & Conc. & -0.006 & -0.010 & 0.005 & -0.007 & 0.018 & 0.048 & 0.004 & -0.001 & -0.031 & -0.007 & -0.005 & 0.035 \\
\hline & Intro. & 0.001 & 0.001 & -0.001 & -0.006 & 0.034 & 0.043 & -0.001 & -0.006 & -0.003 & -0.004 & 0.010 & 0.012 \\
\hline \multirow{3}{*}{ ACL-long } & Abs. & 0.003 & -0.021 & -0.004 & -0.031 & 0.081 & 0.084 & -0.011 & -0.027 & -0.010 & -0.011 & 0.008 & 0.004 \\
\hline & Conc. & 0.010 & -0.003 & 0.003 & -0.015 & 0.079 & 0.087 & 0.000 & 0.003 & 0.020 & 0.014 & 0.045 & 0.028 \\
\hline & Intro. & 0.015 & 0.010 & -0.015 & -0.020 & 0.049 & 0.074 & -0.018 & -0.018 & 0.006 & 0.002 & 0.015 & 0.026 \\
\hline \multirow{3}{*}{ AI-less 10} & Abs. & 0.030 & 0.001 & -0.011 & 0.009 & 0.016 & 0.000 & 0.006 & 0.008 & 0.019 & 0.023 & 0.013 & 0.019 \\
\hline & Conc. & 0.033 & -0.001 & 0.011 & 0.017 & 0.019 & 0.012 & 0.012 & 0.004 & 0.018 & 0.021 & 0.037 & 0.053 \\
\hline & Intro. & 0.030 & 0.025 & -0.009 & -0.001 & 0.007 & 0.012 & 0.027 & 0.018 & 0.008 & 0.014 & 0.021 & 0.039 \\
\hline \multirow{3}{*}{$A I-1013$} & Abs. & 0.038 & 0.028 & 0.005 & -0.001 & -0.003 & -0.025 & 0.004 & -0.007 & 0.014 & 0.010 & 0.023 & 0.047 \\
\hline & Conc. & 0.036 & 0.025 & 0.011 & 0.020 & 0.017 & -0.005 & 0.008 & -0.001 & 0.026 & 0.028 & 0.011 & 0.041 \\
\hline & Intro. & 0.043 & 0.029 & -0.008 & 0.012 & -0.009 & -0.021 & 0.029 & 0.024 & 0.008 & 0.011 & 0.019 & 0.044 \\
\hline \multirow{3}{*}{$A I-1316$} & Abs. & 0.033 & 0.008 & 0.005 & 0.015 & 0.003 & -0.014 & 0.018 & 0.019 & 0.028 & 0.016 & 0.033 & 0.044 \\
\hline & Conc. & 0.040 & 0.019 & 0.004 & 0.011 & 0.012 & 0.004 & 0.022 & 0.011 & 0.016 & 0.010 & 0.030 & 0.033 \\
\hline & Intro. & 0.049 & 0.030 & -0.016 & 0.005 & -0.020 & -0.014 & 0.022 & 0.018 & 0.014 & 0.016 & 0.036 & 0.044 \\
\hline \multirow{3}{*}{$A I-1619$} & Abs. & 0.039 & 0.018 & 0.005 & 0.014 & 0.027 & 0.010 & 0.014 & 0.010 & 0.009 & 0.024 & 0.024 & 0.035 \\
\hline & Conc. & 0.046 & 0.024 & 0.016 & 0.010 & 0.010 & 0.010 & 0.023 & 0.024 & 0.016 & 0.025 & 0.023 & 0.043 \\
\hline & Intro. & 0.045 & 0.035 & -0.011 & 0.011 & -0.002 & -0.002 & 0.032 & 0.030 & 0.006 & 0.032 & 0.019 & 0.043 \\
\hline \multirow{3}{*}{$A I-1925$} & $A b s$. & 0.021 & -0.033 & 0.008 & 0.018 & -0.024 & -0.008 & 0.013 & 0.021 & 0.025 & 0.027 & 0.030 & 0.040 \\
\hline & Conc. & 0.039 & -0.007 & -0.003 & 0.011 & -0.018 & 0.008 & 0.033 & 0.032 & 0.025 & 0.021 & 0.029 & 0.037 \\
\hline & Intro. & 0.040 & 0.020 & -0.027 & -0.008 & -0.047 & -0.013 & 0.035 & 0.036 & 0.015 & 0.022 & 0.035 & 0.050 \\
\hline AI-more 25 & Abs. & 0.050 & 0.016 & -0.004 & 0.008 & -0.040 & -0.062 & 0.039 & 0.031 & 0.024 & 0.028 & 0.037 & 0.038 \\
\hline
\end{tabular}




\begin{tabular}{l|l|l|l|l|l|l|l|l|l|l|l|l|l}
\hline & Conc. & 0.048 & 0.009 & -0.025 & -0.008 & -0.066 & -0.081 & 0.051 & 0.041 & 0.006 & 0.016 & 0.036 & 0.041 \\
\cline { 2 - 22 } & Intro. & 0.052 & 0.025 & -0.052 & -0.034 & -0.085 & -0.117 & 0.063 & 0.060 & 0.014 & 0.020 & 0.039 & 0.037 \\
\hline
\end{tabular}

Table C-6. The Increase metric on each sub-dataset when using different sim-SLNs and different standard summary.

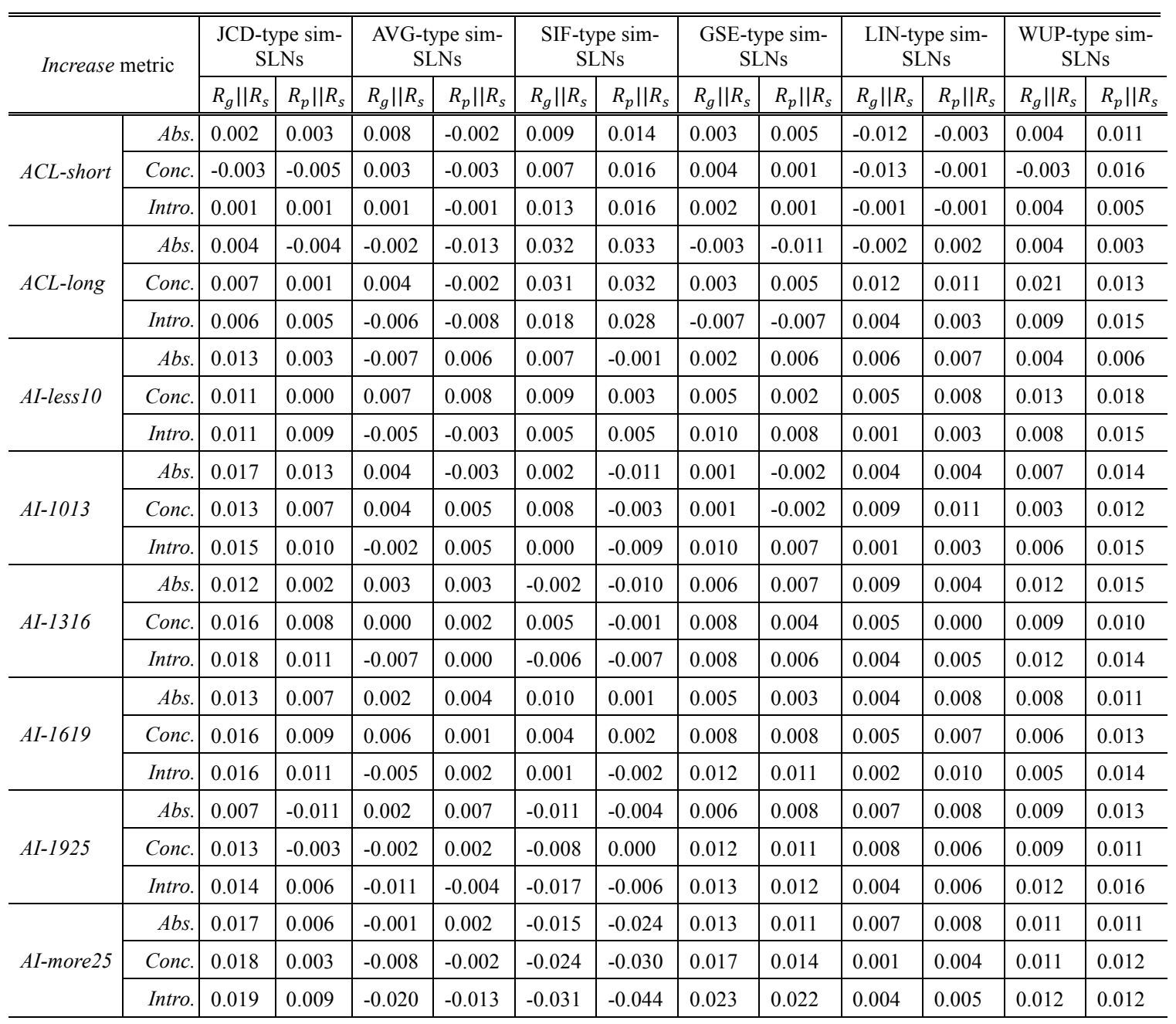

Table C-7. The Increase\% metric on each sub-dataset when using different sim-SLNs and different standard summary.

\begin{tabular}{|c|c|c|c|c|c|c|c|c|c|c|c|c|c|}
\hline \multirow{2}{*}{\multicolumn{2}{|c|}{ Increase $\%$ metric }} & \multicolumn{2}{|c|}{$\begin{array}{c}\text { JCD-type sim- } \\
\text { SLNs } \\
\end{array}$} & \multicolumn{2}{|c|}{$\begin{array}{l}\text { AVG-type sim- } \\
\text { SLNs }\end{array}$} & \multicolumn{2}{|c|}{$\begin{array}{l}\text { SIF-type sim- } \\
\text { SLNs }\end{array}$} & \multicolumn{2}{|c|}{$\begin{array}{l}\text { GSE-type sim- } \\
\text { SLNs } \\
\end{array}$} & \multicolumn{2}{|c|}{$\begin{array}{l}\text { LIN-type sim- } \\
\text { SLNs }\end{array}$} & \multicolumn{2}{|c|}{$\begin{array}{l}\text { WUP-type sim- } \\
\text { SLNs }\end{array}$} \\
\hline & & $R_{g} \| R_{S}$ & $R_{p} \| R_{s}$ & $R_{g} \| R_{s}$ & $R_{p} \| R_{s}$ & $R_{g} \| R_{s}$ & $R_{p} \| R_{s}$ & $R_{g} \| R_{S}$ & $R_{p} \| R_{S}$ & $R_{g} \| R_{S}$ & $R_{p} \| R_{s}$ & $R_{g} \| R_{s}$ & $R_{p} \| R_{s}$ \\
\hline \multirow{3}{*}{$A C L$-short } & Abs. & 0.000 & 0.063 & 0.102 & -0.035 & 0.196 & 0.265 & 0.091 & 0.164 & -0.144 & 0.003 & 0.179 & 0.257 \\
\hline & Conc. & -0.032 & -0.054 & 0.017 & -0.087 & 0.068 & 0.146 & 0.106 & 0.066 & -0.155 & 0.028 & -0.072 & 0.246 \\
\hline & Intro. & 0.009 & 0.004 & 0.053 & 0.025 & 0.111 & 0.141 & 0.067 & 0.046 & -0.010 & 0.004 & 0.046 & 0.050 \\
\hline \multirow{3}{*}{ ACL-long } & Abs. & 0.090 & 0.048 & -0.031 & -0.139 & 0.792 & 0.761 & 0.024 & -0.120 & 0.040 & 0.205 & 0.079 & 0.084 \\
\hline & Conc. & 0.134 & 0.086 & 0.146 & 0.118 & 0.492 & 0.444 & 0.157 & 0.182 & 0.238 & 0.265 & 0.270 & 0.170 \\
\hline & Intro. & 0.053 & 0.064 & -0.026 & -0.048 & 0.150 & 0.241 & -0.061 & -0.045 & 0.061 & 0.078 & 0.133 & 0.203 \\
\hline \multirow{3}{*}{ AI-less 10} & Abs. & 0.140 & 0.115 & -0.140 & 0.185 & 0.084 & -0.042 & 0.013 & 0.149 & 0.109 & 0.078 & 0.015 & 0.101 \\
\hline & Conc. & 0.072 & 0.011 & 0.174 & 0.158 & 0.104 & -0.004 & 0.040 & 0.004 & 0.055 & 0.201 & 0.159 & 0.204 \\
\hline & Intro. & 0.063 & 0.059 & -0.053 & -0.071 & 0.091 & 0.049 & 0.071 & 0.088 & -0.052 & -0.011 & 0.086 & 0.156 \\
\hline
\end{tabular}




\begin{tabular}{|c|c|c|c|c|c|c|c|c|c|c|c|c|c|}
\hline \multirow{3}{*}{$A I-1013$} & Abs. & 0.248 & 0.203 & 0.140 & -0.125 & 0.171 & -0.163 & 0.017 & 0.064 & -0.021 & 0.022 & -0.021 & 0.055 \\
\hline & Conc. & 0.088 & 0.020 & 0.027 & -0.025 & 0.085 & -0.041 & -0.039 & -0.034 & 0.113 & 0.191 & -0.021 & 0.010 \\
\hline & Intro. & 0.073 & 0.034 & 0.024 & 0.044 & 0.066 & -0.050 & 0.031 & 0.008 & -0.026 & 0.004 & 0.018 & 0.082 \\
\hline \multirow{3}{*}{$A I-1316$} & $A b s$. & 0.132 & 0.007 & 0.140 & -0.055 & -0.098 & -0.217 & 0.037 & 0.161 & 0.116 & -0.122 & 0.369 & 0.257 \\
\hline & Conc. & 0.123 & 0.072 & -0.028 & -0.019 & 0.040 & -0.037 & 0.047 & 0.051 & 0.005 & -0.103 & 0.045 & 0.029 \\
\hline & Intro. & 0.110 & 0.064 & -0.063 & -0.043 & -0.005 & -0.070 & 0.032 & 0.028 & 0.009 & -0.005 & 0.050 & 0.057 \\
\hline \multirow{3}{*}{$A I-1619$} & Abs. & 0.106 & 0.084 & 0.029 & -0.006 & 0.214 & -0.129 & 0.062 & 0.066 & 0.222 & 0.185 & 0.058 & 0.022 \\
\hline & Conc. & 0.115 & 0.100 & 0.057 & -0.050 & 0.058 & -0.071 & 0.058 & 0.042 & 0.037 & 0.024 & -0.010 & 0.036 \\
\hline & Intro. & 0.089 & 0.034 & -0.057 & -0.028 & 0.039 & -0.034 & 0.084 & 0.072 & -0.006 & 0.048 & -0.008 & 0.044 \\
\hline \multirow{3}{*}{$A I-1925$} & Abs. & 0.075 & -0.131 & 0.091 & 0.262 & -0.264 & -0.089 & 0.270 & 0.389 & 0.031 & -0.045 & 0.096 & 0.437 \\
\hline & Conc. & 0.088 & -0.055 & -0.053 & -0.023 & -0.094 & -0.087 & 0.123 & 0.074 & 0.076 & 0.019 & 0.024 & 0.061 \\
\hline & Intro. & 0.081 & 0.011 & -0.084 & -0.055 & -0.084 & -0.062 & 0.087 & 0.068 & -0.001 & 0.002 & 0.083 & 0.089 \\
\hline \multirow{3}{*}{ AI-more 25} & Abs. & 0.380 & 0.246 & 0.006 & 0.040 & -0.178 & -0.324 & 0.312 & 0.272 & -0.133 & 0.000 & 0.294 & 0.180 \\
\hline & Conc. & 0.179 & 0.034 & -0.044 & 0.002 & -0.134 & -0.204 & 0.106 & -0.368 & -0.031 & -0.016 & 0.038 & 0.030 \\
\hline & Intro. & 0.140 & 0.052 & -0.108 & -0.087 & -0.139 & -0.204 & 0.141 & 0.137 & -0.008 & -0.020 & 0.044 & 0.048 \\
\hline
\end{tabular}

Table C-8. The Diverge metric on each sub-dataset when using different sim-SLNs and different standard summary.

\begin{tabular}{|c|c|c|c|c|c|c|c|c|c|c|c|c|c|}
\hline \multicolumn{2}{|c|}{ Diverge metric } & \multicolumn{2}{|c|}{$\begin{array}{l}\text { JCD-type sim- } \\
\text { SLNs }\end{array}$} & \multicolumn{2}{|c|}{$\begin{array}{l}\text { AVG-type sim- } \\
\text { SLNs }\end{array}$} & \multicolumn{2}{|c|}{$\begin{array}{l}\text { SIF-type sim- } \\
\text { SLNs }\end{array}$} & \multicolumn{2}{|c|}{$\begin{array}{l}\text { GSE-type sim- } \\
\text { SLNs }\end{array}$} & \multicolumn{2}{|c|}{$\begin{array}{l}\text { LIN-type sim- } \\
\text { SLNs }\end{array}$} & \multicolumn{2}{|c|}{$\begin{array}{l}\text { WUP-type sim- } \\
\text { SLNs }\end{array}$} \\
\hline \multirow{3}{*}{$A C L$-short } & Abs. & 0.014 & 0.023 & 0.060 & -0.013 & 0.066 & & & & -0.084 & -0.022 & 0.034 & 0.089 \\
\hline & Conc. & -0.021 & -0.035 & 0.020 & -0.022 & 0.049 & 0.125 & 0.027 & 0.011 & -0.089 & -0.010 & -0.019 & 0.128 \\
\hline & Intro. & 0.004 & 0.002 & 0.007 & -0.008 & 0.094 & 0.120 & 0.012 & -0.003 & -0.009 & -0.006 & 0.032 & 0.037 \\
\hline \multirow{3}{*}{ ACL-long } & Abs. & 0.030 & -0.028 & -0.015 & -0.086 & 0.278 & 0.281 & -0.024 & -0.077 & -0.012 & 0.023 & 0.028 & 0.022 \\
\hline & Conc. & 0.052 & 0.012 & 0.029 & -0.014 & 0.254 & 0.260 & 0.028 & 0.037 & 0.096 & 0.090 & 0.164 & 0.100 \\
\hline & Intro. & 0.044 & 0.036 & -0.039 & -0.056 & 0.139 & 0.220 & -0.053 & -0.047 & 0.028 & 0.025 & 0.069 & 0.114 \\
\hline \multirow{3}{*}{ AI-less 10} & Abs. & 0.097 & 0.028 & -0.045 & 0.047 & 0.051 & -0.006 & 0.016 & 0.043 & 0.043 & 0.051 & 0.031 & 0.048 \\
\hline & Conc. & 0.085 & 0.002 & 0.051 & 0.059 & 0.064 & 0.024 & 0.035 & 0.012 & 0.040 & 0.061 & 0.098 & 0.138 \\
\hline & Intro. & 0.080 & 0.066 & -0.032 & -0.020 & 0.037 & 0.036 & 0.076 & 0.059 & 0.006 & 0.024 & 0.062 & 0.115 \\
\hline \multirow{3}{*}{$A I-1013$} & Abs. & 0.135 & 0.096 & 0.031 & -0.020 & 0.020 & -0.076 & 0.007 & -0.012 & 0.029 & 0.026 & 0.050 & 0.112 \\
\hline & Conc. & 0.097 & 0.055 & 0.031 & 0.037 & 0.056 & -0.019 & 0.010 & -0.012 & 0.068 & 0.084 & 0.022 & 0.093 \\
\hline & Intro. & 0.114 & 0.070 & -0.012 & 0.036 & 0.001 & -0.061 & 0.071 & 0.053 & 0.010 & 0.025 & 0.044 & 0.115 \\
\hline \multirow{3}{*}{$A I-1316$} & Abs. & 0.093 & 0.018 & 0.022 & 0.022 & -0.011 & -0.064 & 0.042 & 0.054 & 0.067 & 0.028 & 0.091 & 0.118 \\
\hline & Conc. & 0.117 & 0.057 & -0.001 & 0.016 & 0.034 & -0.004 & 0.057 & 0.032 & 0.035 & 0.005 & 0.071 & 0.075 \\
\hline & Intro. & 0.136 & 0.080 & -0.052 & -0.002 & -0.039 & -0.049 & 0.056 & 0.046 & 0.032 & 0.033 & 0.087 & 0.106 \\
\hline \multirow{3}{*}{$A I-1619$} & Abs. & 0.097 & 0.049 & 0.012 & 0.027 & 0.079 & 0.006 & 0.035 & 0.023 & 0.028 & 0.062 & 0.058 & 0.081 \\
\hline & Conc. & 0.123 & 0.070 & 0.043 & 0.009 & 0.032 & 0.012 & 0.058 & 0.055 & 0.036 & 0.054 & 0.047 & 0.096 \\
\hline & Intro. & 0.119 & 0.083 & -0.038 & 0.017 & 0.009 & -0.012 & 0.088 & 0.078 & 0.012 & 0.077 & 0.038 & 0.102 \\
\hline \multirow{3}{*}{ AI-1925 } & Abs. & 0.136 & 0.048 & -0.008 & 0.016 & -0.099 & -0.155 & 0.104 & 0.084 & 0.051 & 0.063 & 0.090 & 0.090 \\
\hline & Conc. & 0.137 & 0.024 & -0.059 & -0.015 & -0.160 & -0.200 & 0.133 & 0.102 & 0.008 & 0.032 & 0.082 & 0.091 \\
\hline & Intro. & 0.147 & 0.066 & -0.135 & -0.094 & -0.212 & -0.287 & 0.177 & 0.169 & 0.027 & 0.036 & 0.092 & 0.089 \\
\hline \multirow{3}{*}{ AI-more 25} & Abs. & 0.051 & -0.076 & 0.018 & 0.053 & -0.073 & -0.025 & 0.043 & 0.063 & 0.053 & 0.057 & 0.069 & 0.099 \\
\hline & Conc. & 0.100 & -0.023 & -0.017 & 0.017 & -0.053 & 0.001 & 0.091 & 0.079 & 0.062 & 0.048 & 0.064 & 0.086 \\
\hline & Intro. & 0.105 & 0.045 & -0.077 & -0.031 & -0.117 & -0.043 & 0.096 & 0.091 & 0.030 & 0.047 & 0.090 & 0.123 \\
\hline
\end{tabular}




\section{C.3 The Role of the Types of Semantic Links in Generating Groups}

Figure C-2, Figure C-3, Figure C-4 and Figure C-5 show more results of the Diverge metric got by using different groups that are generated by different clustering algorithms. The results shown in these figures support the five strategies we proposed about using the is-part-of, sequential, similar-to and cause-effect links to generate groups.

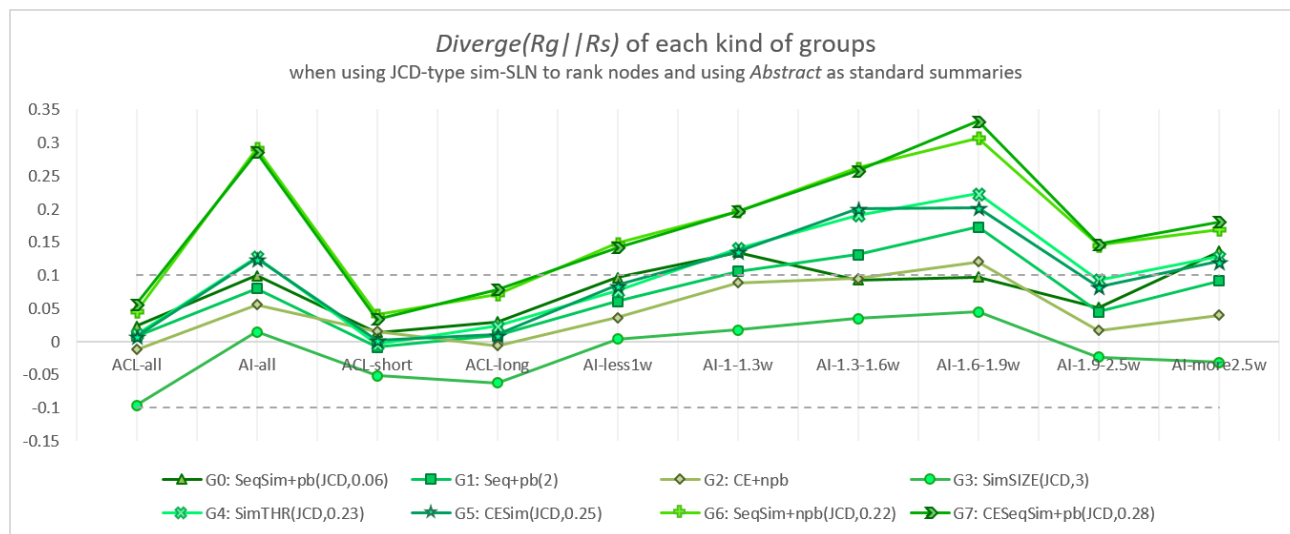

Figure C-2. The Diverge metric results of different kinds of group on all datasets when using JCD-type sim-SLN to rank nodes and using Abstract as standard summaries.

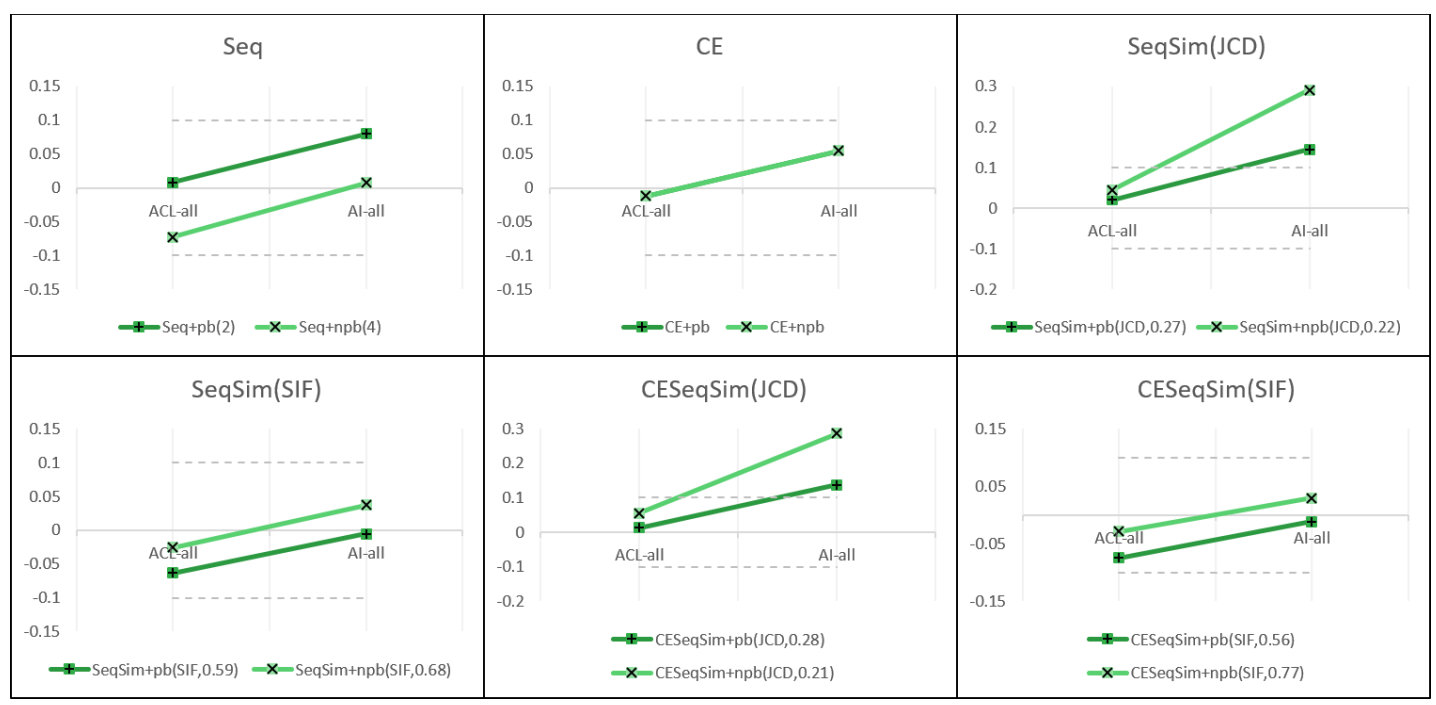

(a) Using ' $+p b$ ' or ' $+n p b$ ' mode in each clustering algorithm.

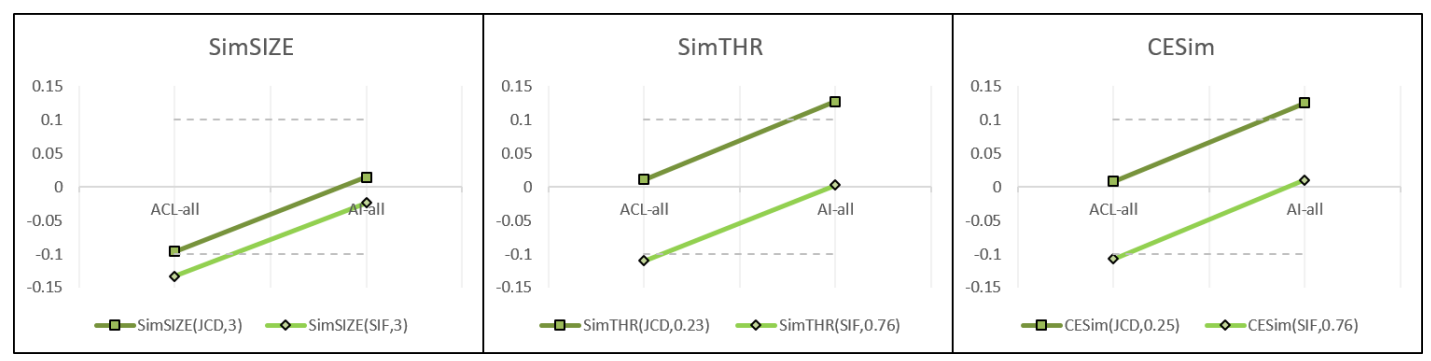

(b) Using JCD-type or SIF-type similar-to link in each clustering algorithm.

Figure C-3. The Diverge metric of each kind of group on the $A C L$-all and AI-all datasets when using JCD-type sim-SLN to rank nodes and using Abstract as standard summaries. 


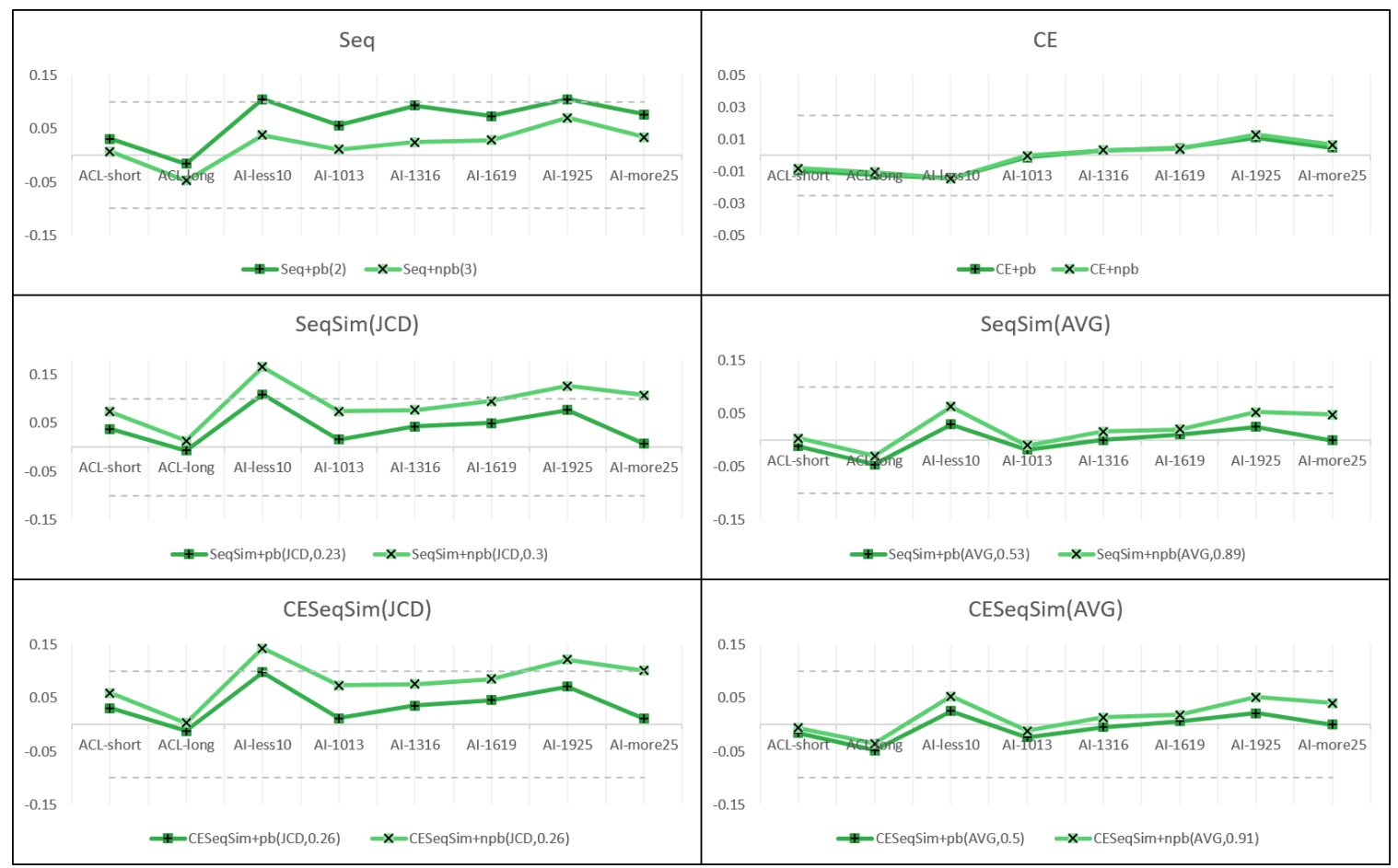

(a) Using ' $+p b$ ' or ' $+n p b$ ' mode in each clustering algorithm.

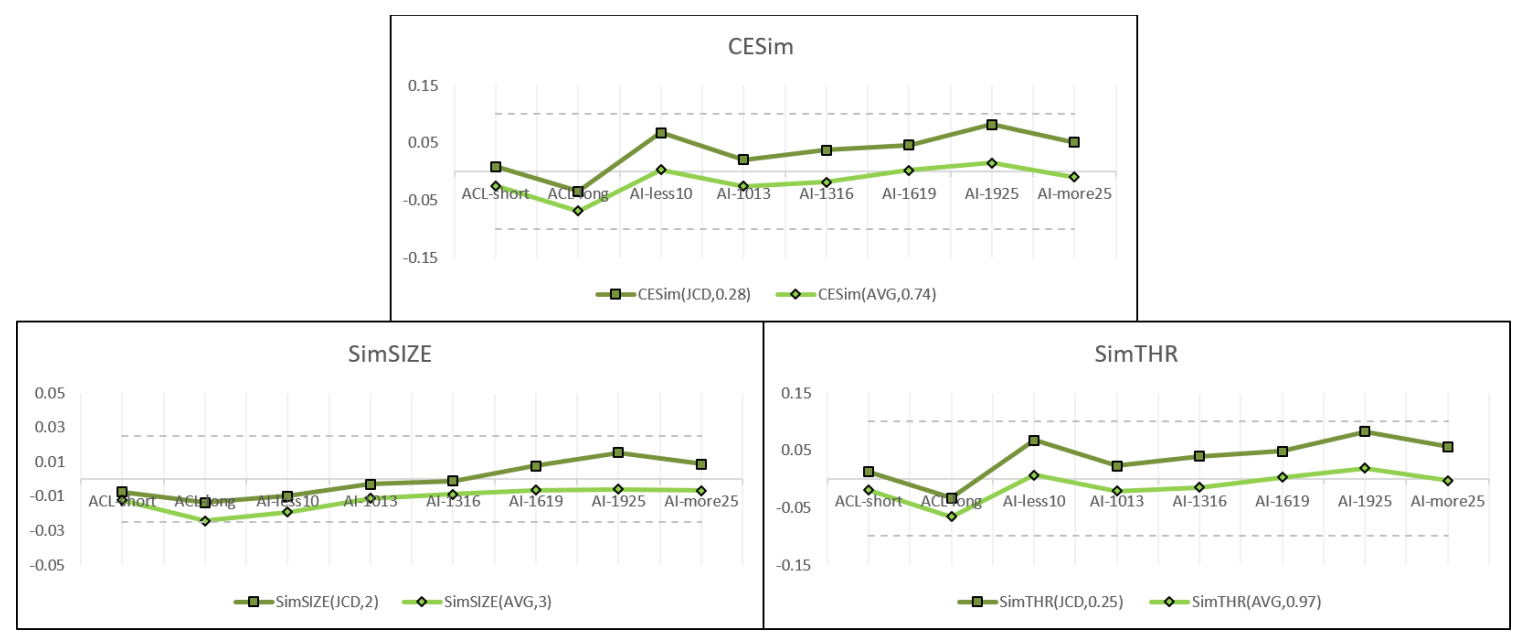

(b) Using JCD-type or AVG-type similar-to link in each clustering algorithm.

Figure C-4. The Diverge metric results of each kind of group on all sub-datasets when using AVG-type sim-SLN to rank and using Abstract as standard summaries 


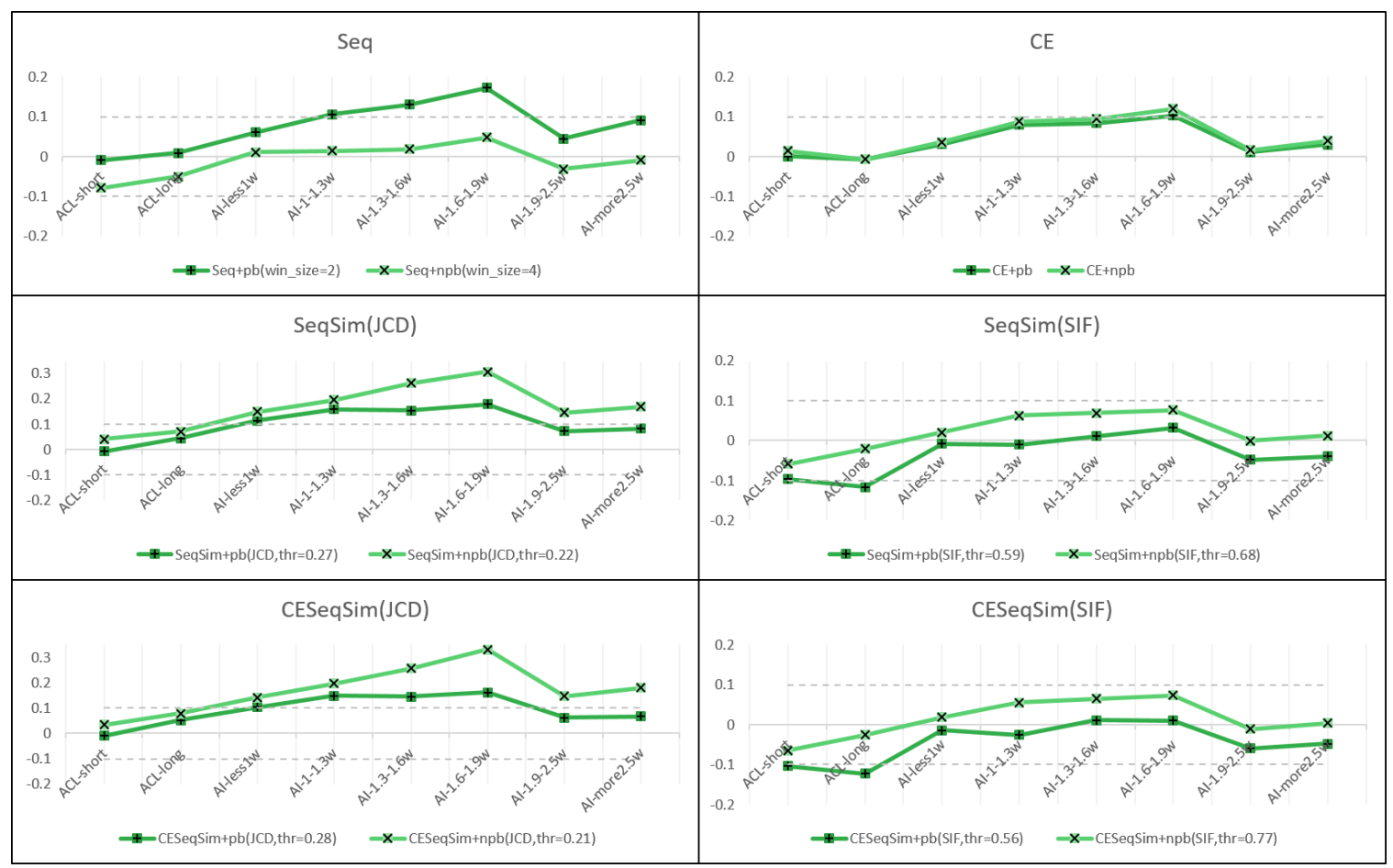

(a) Using ' $+p b$ ' or ' $+n p b$ ' mode in each clustering algorithm.

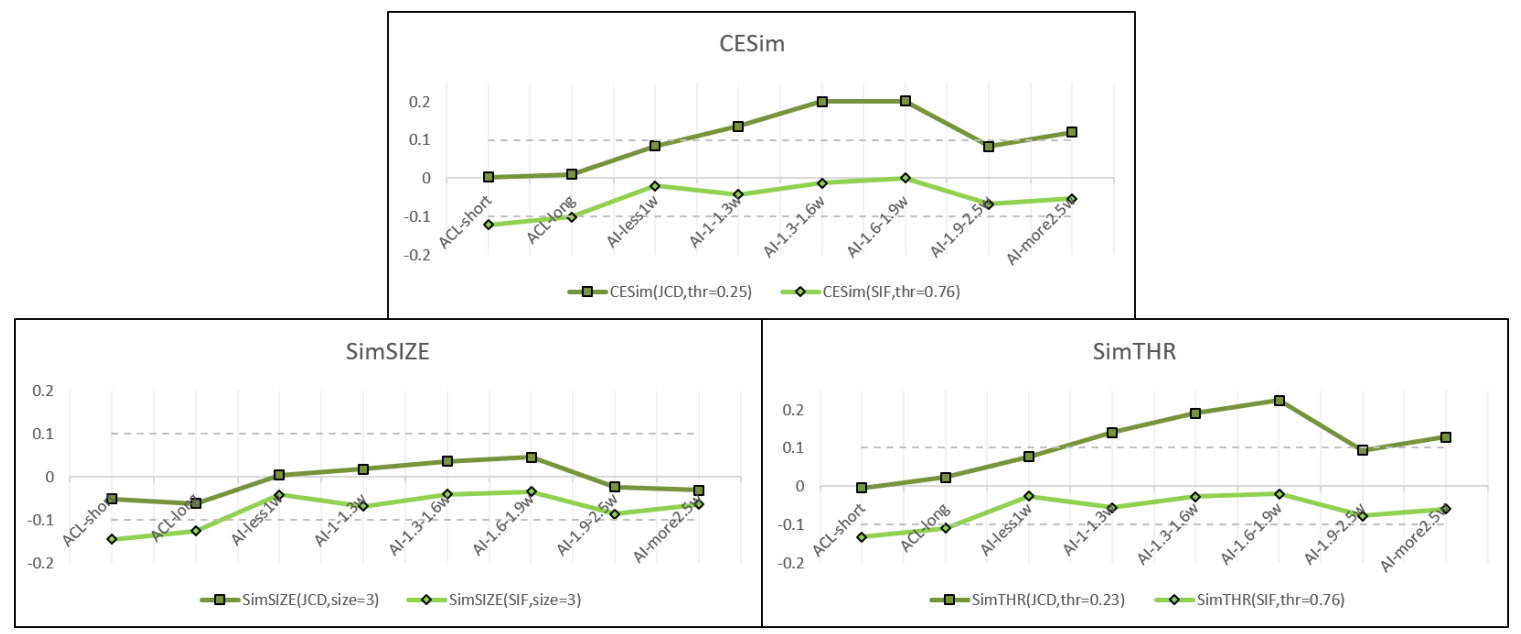

(b) Using JCD-type or SIF-type similar-to link in each clustering algorithm.

Figure C-5. The Diverge values of each kind of group on all sub-datasets when using JCD-type sim-SLN to rank nodes and using $A b-$ stract as standard summaries. 


\section{Appendix D. The Readability Evaluation}

A summary generated by extracting sentences from the source text in isolation usually has low readability, because the context and the original semantic relations of the extracted sentences are lost. Since sentences in the source text are coherent, we propose the following assumption:

Assumption: Extracting larger language units that retain the semantics between sentences in the source text to generate a summary naturally improves the readability of the generated summary.

To verify the above assumption, we randomly select 18 long papers and 17 short papers from the $A C L$-all dataset, and then collect the sentence-based, group-based and paragraph-based summaries for each paper to form a dataset named as Samples. The Samples dataset is further divided into the Samples-long and Samplesshort sub-datasets to study the readability of summaries of different lengths of papers.

We consider that the readability of a summary includes four aspects:

- Sentence integrity, referring each sentence in a summary should be a grammatically complete sentence.

- Referential clarity, referring the anaphors (including pronouns, noun phrases started with a determiner, unfamiliar named entities and unfamiliar abbreviations) within a summary can be resolved correctly.

- Local coherence, referring to any two adjacent sentences should be coherent. The local coherence can be assessed from three aspects:

- Thematic coherence, meaning that two adjacent sentences should talk about the same theme. The theme of a sentence can be reflected by the noun/verb phrases within the sentence.

- Conciseness, meaning that any two adjacent sentences should not express the same meaning. We view the conciseness as an aspect of local coherence instead of emphasizing it on the whole summary because it is reasonable to have some redundancy in a summary to highlight some themes.

- Semantic coherence, meaning that the two adjacent sentences should correctly express a semantic relation when the corresponding cue word/phrase/sentence-pattern occurs. For example, if $s_{1}$ and $s_{2}$ are two adjacent sentences in a summary and $s_{2}$ starts with the word 'However', then there should be a transitional relation between $s_{l}$ and $s_{2}$.

- Structure, referring that the generated summaries should follow the structure that people follow when they write a summary. For example, the structure of summaries for scientific papers should be the purpose $\rightarrow$ the backgroud $\rightarrow$ the methods $\rightarrow$ the results or conclusions.

To date, there is no appropriate metric for evaluating the readability of summaries according to the aspects listed above. Researchers in the field of education and linguistics first put forward some metrics to measure the readability of texts, such as SMOG index[56], automated readability index, Gunning fog index and Flesch reading easy formula [57]. However, these metrics are only applicable in evaluating the manually written articles, because they only depend on the percentage of complex words and the average amount of words in each sentence without considering any aspect of readability listed above. The models introduced in $[58,59]$ aim to evaluate the local coherence. But they just estimate the probability of dependence between any two content words (including verbs, nouns, named entity tags and connectives) to form the score of coherence, without considering the semantic coherence between adjacent sentences, that is, whether adjacent sentences convey a semantic relation such as cause-effect, question-answer, transition, progressive relation, and so on. The model in [60] aims to automatically evaluate the readability of summaries from aspects of grammaticality, coherence and focus. However, the grammaticality evaluated in this work just refers to the frequency of POStag or chunk-tag tri-gram sequence, and the coherence between two adjacent sentences is still evaluated by estimating the relatedness among noun phrases within these two sentences.

We invite two volunteers A and B to manually evaluate the readability of summaries. The referential clarity is evaluated according to the number of dangling anaphors within a summary. Dangling anaphors are those anaphors whose referents cannot be found in the text, and a summary has higher referential clarity if it contains less dangling anaphors. The sentence integrity, the local coherence and the structure of a summary are evaluated together according to the readability rating of a summary, which is an integer from 0 to 5 that is obtained by sum up the scores in Table D-1.

Figure D-2 shows the dangling anaphors and the readability ratings annotated for the generated summaries of a scientific paper. The phrase "the baseline" in the sentence-based summary is labelled as a dangling anaphor since its referent cannot be found in this summary. No dangling anaphor is found in both groupbased and paragraph-based summary. The readability ratings of the group-based summary given by A and B are both 5 , which are higher than the readability ratings of sentence-based and paragraph-based summary. 
All the summaries in the Samples dataset and the manual annotations for evaluating the readability of these summaries can be downloaded from the GitHub ${ }^{1}$.

Table D-1. The aspects and the corresponding score values for calculating the readability rating of a summary.

\begin{tabular}{|c|c|c|c|}
\hline \multicolumn{2}{|c|}{ Aspect } & Condition & Score \\
\hline \multicolumn{2}{|c|}{ Sentence Integrity } & $\begin{array}{l}\text { All sentences are complete sentences. } \\
\text { (Wrong segmentation of sentence and formulas cause incomplete sentences.) }\end{array}$ & 1 \\
\hline \multirow{3}{*}{$\begin{array}{l}\text { Local Cohe- } \\
\text { rence }\end{array}$} & $\begin{array}{l}\text { Thematic co- } \\
\text { herence }\end{array}$ & $\begin{array}{l}\text { More than } 66 \% \text { pairs of adjacent sentences sharing at least one noun/verb phrase } \\
\text { (The parameter } 66 \% \text { is selected according to the observation that more than } 96 \% \\
\text { summaries in the Sample dataset have at least } 3 \text { pairs of adjacent sentences.) }\end{array}$ & 1 \\
\hline & Conciseness & Any two adjacent sentences should not express the same meaning. & 1 \\
\hline & $\begin{array}{l}\text { Semantic co- } \\
\text { herence }\end{array}$ & $\begin{array}{l}\text { Any two adjacent sentences express the correct semantic relations if they contain the } \\
\text { corresponding cue word/phrase/sentence-pattern. } \\
\text { (We require that the question sentence in the summary must be followed by an answer } \\
\text { sentence.) }\end{array}$ & 1 \\
\hline \multicolumn{2}{|l|}{ Structure } & $\begin{array}{l}\text { The sentences within a summary expresses two or more of the purpose, the back- } \\
\text { ground, the methods, the results or conclusions. }\end{array}$ & 1 \\
\hline
\end{tabular}

\begin{tabular}{|c|c|c|}
\hline \multicolumn{3}{|r|}{ Summaries for paper P14-2106 (ACL2014) } \\
\hline & \multicolumn{2}{|c|}{$\begin{array}{l}\text { (0) This paper presents experiments with WordNet semantic classes to improve dependency parsing. } \\
\text { (1) We study the effect of semantic classes in three dependency parsers, using two types of constituency-to-dependency conversions of the } \\
\text { English Penn Treebank. } \\
\text { (2) Overall, we can say that the improvements are small and not significant using automatic POS tags, contrary to previously published } \\
\text { results using gold POS tags. } \\
\text { (3) In addition, we explore parser combinations, showing that the semantically enhanced parsers yield a small significant gain only on the } \\
\text { more semantically oriented LTH treebank conversion. }\end{array}$} \\
\hline 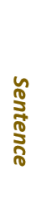 & \multicolumn{2}{|c|}{$\begin{array}{l}\text { (0) This work presents a set of experiments to investigate the use of lexical semantic information in dependency parsing of English } \\
\text { (1) We will apply different types of semantic information to three dependency parsers } \\
\text { (2) Does semantic information in WordNet help dependency parsing found improvements in dependency parsing using MaltParser on } \\
\text { gold POS tags } \\
\text { (3) Different parsers can use semantic information in diverse ways } \\
\text { (4) We will run passer combination experiments with and without semantic information, to determine whether it is useful in the combined } \\
\text { parsers } \\
\text { (5) Extended parsers, adding semantic information to the baselines }\end{array}$} \\
\hline & Dangling Anaphors: [\#5 the baselines] & Readability Rating: (A, 3), (B, 3) ---- Sentence Integrity $=0$ ((5) is incomplete), Structure $=0$ \\
\hline & \multicolumn{2}{|c|}{$\begin{array}{l}\text { (0) This work presents a set of experiments to investigate the use of lexical semantic information in dependency parsing of English } \\
\text { (1) Whether semantics improve parsing is one interesting research topic both on parsing and lexical semantics } \\
\text { (2) Broadly speaking, we can classify the methods to incorporate semantic information into parsers in two systems using static lexical } \\
\text { semantic repositories, such as WordNet or similar ontologies, and systems using dynamic semantic clusters automatically acquired from } \\
\text { corpora } \\
\text { (3) This work has tried to shed light on the contribution of semantic information to dependency parsing }\end{array}$} \\
\hline & Dangling Anaphors: [] & Readability Rating: (A, 5), (B, 5) \\
\hline & \multicolumn{2}{|c|}{$\begin{array}{l}\text { (0) This work presents a set of experiments to investigate the use of lexical semantic information in dependency parsing of English } \\
\text { (1) Whether semantics improve parsing is one interesting research topic both on parsing and lexical semantics } \\
\text { (2) Broadly speaking, we can classify the methods to incorporate semantic information into parsers in two systems using static lexical } \\
\text { semantic repositories, such as WordNet or similar ontologies, and systems using dynamic semantic clusters automatically acquired from } \\
\text { corpora } \\
\text { (3) Is the type of semantic information related to the type of parser }\end{array}$} \\
\hline & Dangling Anaphors: [] & Readability Rating: (A, 4), (B, 4) ---- Semantic Coherence $=0$ ((3) is a question but no answer) \\
\hline
\end{tabular}

Figure D-2. Dangling anaphors and readability ratings in the sentence-based, group-based and paragraph-based summaries of a paper.

Table D-2 lists the weighted kappa coefficients between the two volunteers for the readability ratings, indicating that the readability ratings given by the two annotators are strongly consistent. Table D-3 shows the average amount and the standard deviation of dangling anaphors in the summaries, where the minimum on each dataset is highlighted in bold. Table D-4 shows the average readability ratings and the standard deviation of the summaries, where the maximum on each dataset is highlighted in bold.

From Table D-3 and Table D-4, we can see that both the group-based summary and the paragraph-based summary contain less dangling anaphors and get higher readability ratings than the sentence-based summary, and the group-based summary contains a similar amount of dangling anaphors and gets similar readability ratings as the paragraph-based summary. This suggests that summaries generated by extracting larger language units have better readability than summaries generated by extracting sentences.

\footnotetext{
${ }^{1}$ https://github.com/Angela7126/Group_For_Summarization
} 
Comparing the dangling anaphors statistics on the Samples-long and Samples-short sub-datasets in Table D-3, we find than the number of dangling anaphors contained in the group-based or paragraph-based summary decreases as the source text becomes longer, while the amount of dangling anaphors contained in the sentence-based summary remains the same.

Comparing the average of readability ratings on the Samples-long and Samples-short sub-datasets in Table D-4, we find that the average ratings of the sentence-based, group-based and paragraph-based summary all decline as the source texts become longer. However, the average readability ratings of the group-based and paragraph-based summary decrease less than the average readability rating of sentence-based summary, showing the performance of larger language units in maintaining the readability of the generated summaries when the source text becomes longer.

Based on the above analysis, we can say that the readability of summaries composed by larger language units is better than the readability of the sentence-based summary especially when the source texts become longer, verifying the assumption we proposed in the beginning.

Table D-2. The weighted kappa coefficients between the readability ratings given by the two volunteers.

\begin{tabular}{c|c|c|c}
\hline \hline & sentence-based & group-based & paragraph-based \\
\hline \hline Weighted Kappa & 0.792 & 0.749 & 0.747 \\
\hline
\end{tabular}

Table D-3. The average amount of dangling anaphors in the summaries composed by sentence, group or paragraph.

\begin{tabular}{c|c|c|c}
\hline \hline Dataset & sentence-based & group-based & paragraph-based \\
\hline \hline Samples & $4.4 \pm 2.23$ & $3.06 \pm 1.88$ & $\mathbf{3 . 0 3} \pm 2.54$ \\
\hline Samples-long & $4.44 \pm 2.14$ & $2.94 \pm 1.68$ & $\mathbf{2 . 6 7} \pm 1.63$ \\
\hline Samples-short & $4.35 \pm 2.32$ & $\mathbf{3 . 1 8} \pm 2.06$ & $3.41 \pm 3.18$ \\
\hline
\end{tabular}

Table D-4. The average readability ratings of the summaries composed by sentence, group or paragraph.

\begin{tabular}{c|c|c|c}
\hline \hline Dataset & sentence-based & group-based & paragraph-based \\
\hline \hline Samples & $2.0 \pm 1.23$ & $\mathbf{3 . 2 9} \pm 1.24$ & $3.23 \pm 1.26$ \\
\hline Samples-long & $1.78 \pm 1.27$ & $3.08 \pm 1.23$ & $3.08 \pm 1.23$ \\
\hline Samples-short & $2.24 \pm 1.14$ & $\mathbf{3 . 5} \pm 1.22$ & $3.38 \pm 1.26$ \\
\hline
\end{tabular}

\section{Supplementary References}

[56] Mc Laughlin, G. Harry, SMOG grading-a new readability formula, Journal of Reading, 12 (8) (1969) 639-646.

[57] J.P. Kincaid, R.P. Fishburne, R.L. Rogers, B.S. Chissom, Derivation of new readability formulas (automated readability index, fog count, and flesch reading ease formula) for navy enlisted personnel, Naval Air Station Memphis Research Branch Report, (1975).

[58] R. Barzilay, M. Lapata, Modeling local coherence: An entity-based approach, Computational Linguistics, 34 (1) (2008) 1-34.

[59] H. Nishikawa, T. Hasegawa, Y. Matsuo, G. Kikui, Optimizing informativeness and readability for sentiment summarization, in: Proceedings of the ACL 2010 conference short papers, 2010, pp. 325330.

[60] R. Vadlapudi, R. Katragadda, On automated evaluation of readability of summaries: Capturing grammaticality, focus, structure and coherence, in: Proceedings of the NAACL HLT 2010 student research workshop, 2010, pp. 7-12. 


\section{Appendix E. The Extensions of Our Previous Work}

This paper extends our previous work ${ }^{1}$ on the experimental datasets, the semantic links for constructing SLNs, the clustering algorithms for generating groups, and the conclusions of the research. Table E-1 lists all the extensions.

Table E-1. The extensions of our previous work.

\begin{tabular}{|c|c|c|}
\hline & Previous Work & Extensions in this paper \\
\hline $\begin{array}{l}\text { Experimental } \\
\text { dataset }\end{array}$ & $\begin{array}{l}\text { The } A C L \text {-all dataset: it contains } 173 \text { conference } \\
\text { papers ( } 88 \text { long papers and } 85 \text { short papers) col- } \\
\text { lected from the proceedings of ACL } 2014 \text {. } \\
\text { The } A C L \text {-short and } A C L \text {-long datasets: these are } \\
\text { the subsets of the } A C L \text {-all dataset to discuss the } \\
\text { performance of group in extractive summarization } \\
\text { on different length of source text. } \\
\text { The Sample dataset: it contains } 105 \text { summaries of } \\
35 \text { papers for comparing the readability of the } \\
\text { summaries composed by different language units. }\end{array}$ & $\begin{array}{l}\text { The AI-all dataset: it contains } 372 \text { journal papers collected } \\
\text { from the Artificial Intelligence. The length of papers ranges } \\
\text { from } 2542 \text { words to } 52833 \text { words. } \\
\text { The AI-less } 10, A I-1013, A I-1316, A I-1619, A I-1925 \text { and } A I \text { - } \\
\text { more } 25 \text { : these are the subsets of the } A I-\text {-all dataset to discuss } \\
\text { the performance of group in extractive summarization on } \\
\text { different length of source text. }\end{array}$ \\
\hline $\begin{array}{l}\text { Semantic links } \\
\text { for constructing } \\
\text { SLN }\end{array}$ & $\begin{array}{l}\text { The sequential link: the appearance order of sen- } \\
\text { tences in the source text. } \\
\text { The is-part-of link: the subordination between } \\
\text { each sentence to its paragraph. } \\
\text { The similar-to link: Jaccard distance (JCD) is used } \\
\text { to calculate the similarity between two language } \\
\text { units. }\end{array}$ & $\begin{array}{l}\text { The similar-to link: Jaccard distance (JCD), average word } \\
\text { embedding (AVG), smooth inverse frequency (SIF), Google } \\
\text { sentence encoder (GSE), wup-similarity on WordNet } \\
\text { (WUP), and lin-similarity on WordNet (LIN) are used to } \\
\text { calculate the similarity between two language units. } \\
\text { The cause-effect link: a pattern-based algorithm }{ }^{2} \text { is used to } \\
\text { extract the explicit cause-effect links between sentences. }\end{array}$ \\
\hline $\begin{array}{l}\text { Clustering algo- } \\
\text { rithms for gen- } \\
\text { erating groups }\end{array}$ & - $\operatorname{SeqSim}+p b\left(J C D, t h r \in R^{+}\right)$ & 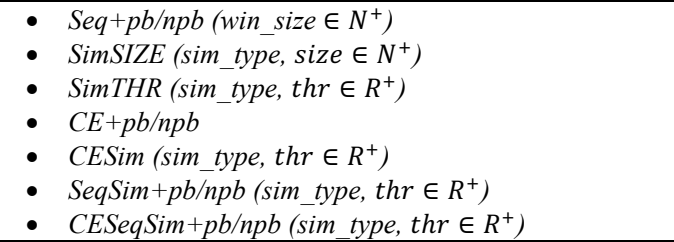 \\
\hline Conclusions & $\begin{array}{l}\text { Conclusion 1: Comparing with the summaries } \\
\text { composed by sentences, the summaries composed } \\
\text { by larger language units have similar ROUGE } \\
\text { scores but have better readability } \\
\text { Conclusion 2: Using a group of sentences is more } \\
\text { effective than using sentence and paragraph. } \\
\text { Conclusion 3: The quality of summaries com- } \\
\text { posed by group becomes better when the average } \\
\text { length of the source texts increases. }\end{array}$ & $\begin{array}{l}\text { Conclusion 1: summaries composed by group or paragraph } \\
\text { tend to contain more key words or phrases than summaries } \\
\text { composed by sentence. } \\
\text { Conclusion 2: summaries composed by group contain more } \\
\text { key words or phrases than those based on paragraph, espe- } \\
\text { cially when the average length of source texts is between } \\
7,000 \text { and 17,000 words. } \\
\text { Strategy 1: The is-part-of link is a suitable clustering con- } \\
\text { straint when only using the sequential link to generate } \\
\text { groups, while in other cases of using the is-part-of link as a } \\
\text { clustering constraint has no effect or even has an adverse } \\
\text { effect for generating groups. } \\
\text { Strategy 2: The lexical-based similar-to link is more suita- } \\
\text { ble for generating groups, while the embedding-based sim- } \\
\text { ilar-to link is more suitable for ranking groups. } \\
\text { Strategy 3: When only using the similar-to link to generate } \\
\text { groups, it is better not to restrict the number of sentences } \\
\text { within each group but to restrict the degree of similar-to be- } \\
\text { tween sentences within each group. } \\
\text { Strategy 4: The sequential link is well suitable to be used } \\
\text { together with the similar-to link for generating groups. } \\
\text { Strategy 5: The cause-effect link improves the readability of } \\
\text { the group-based summary without reducing the amount of } \\
\text { key words/phrases contained in the summaries. }\end{array}$ \\
\hline
\end{tabular}

\footnotetext{
${ }^{1}$ M. Cao, H. Zhuge, What size of language unit is more appropriate for text summarization?, in: Proceedings of the $14^{\text {th }}$ International Conference on Semantics, Knowledge and Grids (SKG), 2018, pp. 196-202.

${ }^{2}$ M. Cao, X. Sun, H. Zhuge, The contribution of cause-effect link to representing the core of scientific paper -- The role of Semantic Link Network, PloS one, 13 (6) (2018) e 0199303.
} 